TRANSACTIONS OF THE

AMERICAN MATHEMATICAL SOCIETY

Volume 364, Number 10, October 2012, Pages 5311-5368

S 0002-9947(2012)05667-4

Article electronically published on May 30, 2012

\title{
HOCHSCHILD (CO)HOMOLOGY OF THE SECOND KIND I
}

\author{
ALEXANDER POLISHCHUK AND LEONID POSITSELSKI
}

\begin{abstract}
We define and study the Hochschild (co)homology of the second kind (known also as the Borel-Moore Hochschild homology and the compactly supported Hochschild cohomology) for curved DG-categories. An isomorphism between the Hochschild (co)homology of the second kind of a CDG-category $B$ and the same of the DG-category $C$ of right CDG-modules over $B$, projective and finitely generated as graded $B$-modules, is constructed.

Sufficient conditions for an isomorphism of the two kinds of Hochschild (co)homology of a DG-category are formulated in terms of the two kinds of derived categories of DG-modules over it. In particular, a kind of "resolution of the diagonal" condition for the diagonal CDG-bimodule $B$ over a CDG-category $B$ guarantees an isomorphism of the two kinds of Hochschild (co)homology of the corresponding DG-category $C$. Several classes of examples are discussed. In particular, we show that the two kinds of Hochschild (co)homology are isomorphic for the DG-category of matrix factorizations of a regular function on a smooth affine variety over a perfect field provided that the function has no other critical values but zero.
\end{abstract}

\section{Contents}

Introduction

1. CDG-categories and QDG-functors

2. $\quad$ Ext and Tor of the second kind

3. Derived categories of the second kind

4. Examples

References

\section{INTRODUCTION}

CDG-algebras (where "C" stands for "curved") were introduced in connection with nonhomogeneous Koszul duality in [13. Several years earlier (what we would now call) $A_{\infty}$-algebras with curvature were considered in [3] as natural generalizations of the conventional $A_{\infty}$-algebras. In fact, 3 appears to be the first paper where the Hochschild (and even cyclic) homology of curved algebras was discussed.

Recently, interest in these algebras was rekindled by their connection with the categories of matrix factorizations [18, 2, 12, 1, 22. In these studies the beginnings of the theory of Hochschild (co)homology for CDG-algebras have emerged. The aim of the present paper is to work out the foundations of the theory on the basis of the general formalism of derived categories of the second kind as developed in the

Received by the editors October 15, 2010.

2010 Mathematics Subject Classification. Primary 16E40; Secondary 18G10, 18G15, 18E30, $13 \mathrm{D} 99$. 
second author's paper [15. The terminology, as well as the notion of a differential derived functor of the second kind, which is relevant here, go back to the classical paper [5].

The subtle but crucial difference between the differential derived functors of the first and the second kinds lies in the way one constructs the totalizations of bicomplexes: one can take either direct sums or direct products along the diagonals. The construction of the differential Tor and Ext of the first kind, which looks generally more natural at first glance, leads to trivial functors in the case of a CDG-algebra with nonzero curvature over a field. So does the (familiar) definition of Hochschild (co)homology of the first kind.

On the other hand, with a CDG-algebra $B$ one can associate the DG-category $C$ of right CDG-modules over $B$, projective and finitely generated as graded $B$ modules. For the DG-category $C$, the Hochschild (co)homology of the first kind makes perfect sense. The main problem that we address in this paper is the problem of comparison between the Hochschild (co)homology of the first kind of the DGcategory $C$ and the Hochschild (co)homology of the second kind of the original CDG-algebra $B$ (defined using the differential Tor/Ext of the second kind).

We proceed in two steps: first, compare the Hochschild (co)homology of the second kind for $B$ and $C$, and then deal with the two kinds of Hochschild (co)homology of $C$. The first step is relatively easy: our construction of an isomorphism works, at least, for any CDG-algebra $B$ over a field $k$ (see Section 2.6). However, a trivial counterexample shows that the two kinds of Hochschild (co)homology of $C$ are not isomorphic in general (see Section 4.9). There are natural maps between the two kinds of Hochschild (co)homology, though. A sufficient condition for these maps to be isomorphisms is formulated in terms of the derived categories of the second kind of CDG-bimodules over $B$. In the maximal generality that we have been able to attain, this is a kind of "resolution of the diagonal" condition for the CDG-bimodule $B$ over $B$ (see Theorems 3.5. C-D and Corollaries 4.6, B, 4.7, B, and 4.8, A).

Let us say a few more words about the first step. There is no obvious map between the Hochschild complexes of $B$ and $C$, so one cannot directly compare their cohomology. Instead, we construct a third complex (both in the homological and the cohomological versions) endowed with natural maps from/to these two complexes, and show that these maps are quasi-isomorphisms. To obtain the intermediate complex, we embed both $B$ and $C$ into a certain larger differential category.

The idea of these embeddings goes back to A. Schwarz's work [19. The starting observation is that a CDG-algebra is not a CDG-module over itself in any natural way (even though it is naturally a CDG-bimodule over itself). It was suggested in [19], however, that one can relax the conditions on differential modules over CDG-algebras (called "Q-algebras" in [19]), thereby making the modules carry their own curvature endomorphisms. In recognition of A. Schwarz's vision, we partly borrow his terminology by calling such modules QDG-modules.

Any CDG-algebra is naturally both a left and a right QDG-module over itself. While CDG-modules form a DG-category, QDG-modules form a CDG-category. Both a CDG-algebra $B$ (considered as a CDG-category with a single object) and the DG-category $C$ of CDG-modules over it embed naturally into the CDG-category $D$ of QDG-modules over $B$, so the Hochschild complex of $D$ provides an intermediate object for comparison between the Hochschild complexes of $B$ and $C$. 
Now let us turn to the second step. The (conventional) derived category of DGmodules over a DG-algebra is defined as the localization of the homotopy category of DG-modules by the class of quasi-isomorphisms or, equivalently, by the thick subcategory of acyclic DG-modules. This does not make sense for CDG-modules, since their differentials have nonzero squares, so their cohomology cannot be defined. Indeed, the subcategory of acyclic DG-modules is not even invariant under CDG-isomorphisms between DG-algebras [15, Examples 9.4].

The definition of the derived categories of the second kind, various species of which are called the coderived, the contraderived, the absolute derived, and the complete derived categories, for DG- and CDG-modules is not based on any notion of cohomology of a differential module. Rather, the classes of coacyclic, contraacyclic, absolutely acyclic, and completely acyclic CDG-modules are built up starting from short exact sequences of CDG-modules (with closed morphisms between them).

For reasons related to the behavior of tensor products with respect to infinite direct sums and products of vector spaces, the derived categories and functors of the second kind work better for coalgebras than for algebras, even though one is forced to use them for algebras if one is interested in curved algebras and modules. (For derived categories and functors of the first kind, it is the other way.) That is why one has to impose additional conditions like finiteness of homological dimension, Noetherianness, etc., on the underlying graded algebras of one's CDG-algebras in order to make the derived categories of the second kind well-behaved and the relation between them and the derived functors of the second kind working properly. We did our best to make such additional conditions as weak as possible in this paper, but the price of generality is technical complexity.

Unlike the Tor and Ext, the Hochschild (co)homology is essentially an invariant of a pair (a field or commutative ring and an algebra over it). It is not preserved when the ground field or ring is changed. In this paper, we always work over an arbitrary commutative ring $k$ or a commutative ring of finite homological dimension, as needed. The only exceptions are some examples depending on the Koszul duality results from [15], which are established only over a field. Working over a commutative ring involves all kinds of $k$-flatness or $k$-projectivity conditions that need to be imposed on the algebras and modules, both in order to define the Hochschild (co)homology and to compute various (co)homology theories in terms of standard complexes.

Recent studies of the categories of matrix factorizations and of the associated CDG-algebras showed the importance of developing the relevant homological algebra using only $\mathbb{Z} / 2$-grading (as opposed to the conventional $\mathbb{Z}$-grading). In this paper we work with CDG-algebras and CDG-categories graded by an arbitrary abelian group $\Gamma$ endowed with some additional data that is needed to define $\Gamma$-graded complexes and perform operations with them. The behavior of our (co)homology theories with respect to a replacement of the grading group $\Gamma$ is discussed in detail (see Section 2.51).

We exhibit several classes of examples of DG-algebras and DG-categories for which the two kinds of Tor, Ext, and Hochschild (co)homology coincide. These examples roughly correspond to the classes of DG-algebras for which the derived categories of the first and second kind are known to coincide [15, Section 9.4]. In particular, one of these classes is that of the DG-categories that are cofibrant with respect to G. Tabuada's model category structure (see Section 4.4). 
Examples of CDG-algebras $B$ such that the two kinds of Tor and Ext for the corresponding DG-category $C$ of CDG-modules over $B$, finitely generated and projective as graded $B$-modules, are known to coincide are fewer; and examples when we can show that the two kinds of Hochschild (co)homology for this DG-category $C$ coincide are fewer still. Among the former are all the CDG-rings $B$ whose underlying graded rings are Noetherian of finite homological dimension (see Section 4.7). In the latter class we have some CDG-algebras over fields admitting Koszul filtrations of finite homological dimension (see Section 4.6), curved commutative local algebras describing germs of isolated hypersurface singularities (due to the results of [2]), and curved commutative smooth algebras over perfect fields with the curvature function having no other critical values but zero (due to the recent results of [8]; see Section 4.8).

Our discussion of the Hochschild (co)homology of the DG-categories of matrix factorizations is finished in Section 4.10, where we show that the Hochschild (co)homology of the second kind of the DG-category of matrix factorizations over a smooth affine variety over an algebraically closed field of characteristic zero is isomorphic to the direct sum of the Hochschild (co)homology of the first kind of the similar DG-categories corresponding to all the critical values of the potential.

We are grateful to Anton Kapustin, Ed Segal, Daniel Pomerleano, Kevin Lin, and Junwu Tu for helpful conversations. The first author was partially supported by the NSF grant DMS-1001364. The second author was partially supported by a grant from the P. Deligne 2004 Balzan prize, a Simons Foundation grant, and an RFBR grant.

\section{CDG-CATEGories And QDG-Functors}

This section is written in the language of CDG-categories. Expositions in the generality of CDG-rings, which might be somewhat more accessible to an inexperienced reader, can be found in [13, 15, 19]. For a discussion of DG-categories, we refer to [6], 21, and [15, Section 1.2].

1.1. Grading group. Let $\Gamma$ be an abelian group endowed with a symmetric bilinear form $\sigma: \Gamma \times \Gamma \longrightarrow \mathbb{Z} / 2$ and a fixed element $\mathbf{1} \in \Gamma$ such that $\sigma(\mathbf{1}, \mathbf{1})=1 \bmod 2$. We will use $\Gamma$ as the group of values for the gradings of our complexes. The differentials will raise the degree by $\mathbf{1}$, and signs such as $(-1)^{\sigma(a, b)}$ will appear in the sign rules.

For example, in the simplest cases one may have $\Gamma=\mathbb{Z}, \mathbf{1}=1$, and $\sigma(a, b)=$ $a b \bmod 2$ for $a, b \in \Gamma$, or, alternatively, $\Gamma=\mathbb{Z} / 2, \quad \mathbf{1}=1 \bmod 2$, and $\sigma(a, b)=a b$. One can also take $\Gamma$ to be any additive subgroup of $\mathbb{Q}$ containing $\mathbb{Z}$ and consisting of fractions with odd denominators, $\mathbf{1}=1$, and $\sigma(a, b)=a b \bmod 2$. Of course, it is also possible that $\Gamma=\mathbb{Z}^{d}$ for any finite or infinite $d$, etc. When working over a commutative ring $k$ containing the field $\mathbb{F}_{2}$, we will not need the form $\sigma$, and so $\Gamma=\mathbb{Q}$ or $\Gamma=0$ become admissible choices as well.

From now on, we will assume a grading group data $(\Gamma, \sigma, \mathbf{1})$ to be fixed. When appropriate, we will identify the integers with their images under the natural map $\mathbb{Z} \longrightarrow \Gamma$ sending 1 to $\mathbf{1}$ without presuming this map to be injective and will denote $\sigma(a, b)$ simply by $a b$ for $a, b \in \Gamma$. So we will write simply 1 instead of $\mathbf{1}$, etc. This map $\mathbb{Z} \longrightarrow \Gamma$ will also be used when constructing the total complexes of polycomplexes, some of whose gradings are indexed by the integers and others by 
elements of the group $\Gamma$. Conversely, to any $a \in \Gamma$ one assigns the class $\sigma(\mathbf{1}, a) \in$ $\mathbb{Z} / 2$, which we will denote simply by $a$ in the appropriate contexts.

1.2. CDG-categories. A $C D G$-category $C$ is a category whose sets of morphisms $\operatorname{Hom}_{C}(X, Y)$ are $\Gamma$-graded abelian groups (i.e., $C$ is a $\Gamma$-graded category) endowed with homogeneous endomorphisms $d: \operatorname{Hom}_{C}(X, Y) \longrightarrow \operatorname{Hom}_{C}(X, Y)$ of degree 1 and fixed elements $h_{X} \in \operatorname{Hom}_{C}(X, X)$ of degree 2 for all objects $X, Y \in C$. The endomorphisms $d$ are called the differentials, and the elements $h_{X}$ are called the curvature elements. The following equations have to be satisfied: $d(f g)=$ $d(f) g+(-1)^{|f|} f d(g)$ for any composable homogeneous morphisms $f$ and $g$ in $C$ of the degrees $|f|$ and $|g| \in \Gamma, \quad d^{2}(f)=h_{Y} f-f h_{X}$ for any morphism $f: X \longrightarrow Y$ in $C$, and $d\left(h_{X}\right)=0$ for any object $X \in C$.

The simplest example of a CDG-category is the category $\operatorname{Pre}(A)$ of precomplexes over an additive category $A$. The objects of $\operatorname{Pre}(A)$ are $\Gamma$-graded objects $X$ in $A$ endowed with an endomorphism $d_{X}: X \longrightarrow X$ of degree 1 . The $\Gamma$-graded abelian group of morphisms $\operatorname{Hom}_{\operatorname{Pre}(A)}(X, Y)$ is the group of homogeneous morphisms $X \longrightarrow Y$ of $\Gamma$-graded objects. The differentials $d: \operatorname{Hom}(X, Y) \longrightarrow \operatorname{Hom}(X, Y)$ are given by the rule $d(f)=d_{Y} f-(-1)^{|f|} f d_{X}$, and the curvature elements are $h_{X}=d_{X}^{2}$. In particular, when $A=A b$ is the category of abelian groups, we obtain the CDG-category of precomplexes of abelian groups $\operatorname{Pre}(A b)$.

A CDG-category with a single object is another name for a $C D G$-ring. A CDGring $(B, d, h)$ is a $\Gamma$-graded ring $B$ endowed with an odd derivation $d$ of degree 1 and a curvature element $h \in B^{2}$ such that $d^{2}(b)=[h, b]$ for any $b \in B$ and $d(h)=0$.

An isomorphism between objects $X$ and $Y$ of a CDG-category $C$ is (an element of) a pair of morphisms $i: X \longrightarrow Y$ and $j: Y \longrightarrow X$ of degree 0 such that $j i=\mathrm{id}_{X}$, $i j=\mathrm{id}_{Y}$, and $d(i)=0=d(j)$; any one of the latter two equations implies the other one. It also follows that $j h_{Y} i=h_{X}$.

Let $X$ be an object of a CDG-category $C$ and $\tau \in \operatorname{Hom}_{C}(X, X)$ be its homogeneous endomorphism of degree 1 . An object $Y \in C$ is called the twist of an object $X$ with an endomorphism $\tau$ (the notation: $Y=X(\tau))$ if homogeneous morphisms $i: X \longrightarrow Y$ and $j: Y \longrightarrow X$ of degree 0 are given such that $j i=\mathrm{id}_{X}, i j=\mathrm{id}_{Y}$, and $j d(i)=\tau$. In this case one has $j h_{Y} i=h_{X}+d \tau+\tau^{2}$. For any object $X \in C$ and an element $n \in \Gamma$, an object $Y \in C$ is called the shift of $X$ with the grading $n$ (the notation: $Y=X[n]$ ) if homogeneous morphisms $i: X \longrightarrow Y$ and $j: Y \longrightarrow X$ of the degrees $n$ and $-n$, respectively, are given such that $j i=\operatorname{id}_{X}, i j=\mathrm{id}_{Y}$, and $d(i)=0=d(j)$. In this case one has $j h_{Y} i=h_{X}$.

An object $X \in C$ is called the direct sum of a family of objects $X_{\alpha} \in C$ if homogeneous morphisms $i_{\alpha}: X_{\alpha} \longrightarrow X$ of degree 0 are given such that the induced map $\operatorname{Hom}_{C}(X, Y) \longrightarrow \prod_{\alpha} \operatorname{Hom}_{C}\left(X_{\alpha}, Y\right)$ is an isomorphism of $\Gamma$-graded abelian groups for any object $Y \in C$, and $d i_{\alpha}=0$. In this case one has $h_{X} i_{\alpha}=i_{\alpha} h_{X_{\alpha}}$, so the endomorphism $h_{X}$ corresponds to the family of morphisms $i_{\alpha} h_{X_{\alpha}}$ under the above isomorphism for $Y=X$. The (direct) product of a family of objects is defined in the dual way. An object $X$ is the direct sum of a finite family of objects $X_{\alpha} \in C$ if and only if it is their direct product. Of course, the notions of a shift and a direct sum/product of objects make sense in a (nondifferential) $\Gamma$-graded category, too; one just drops the conditions involving $d$ and $h$.

Twists, shifts, direct sums, and products of objects of a CDG-category are unique up to a unique isomorphism whenever they exist.

A DG-category is a CDG-category in which all the curvature elements are zero. 
The opposite $C D G$-category to a CDG-category $C$ is constructed as follows. The class of objects of $C^{\text {op }}$ coincides with the class of objects of $C$. For any objects $X, Y \in C$ the $\Gamma$-graded abelian group $\operatorname{Hom}_{C^{\mathrm{op}}}\left(X^{\mathrm{op}}, Y^{\mathrm{op}}\right)$ is identified with $\operatorname{Hom}_{C}(Y, X)$, and the differential $d^{\text {op }}$ on this group coincides with $d$. The composition of morphisms in $C^{\text {op }}$ differs from that in $C$ by the sign rule, $f^{\text {op }} g^{\text {op }}=$ $(-1)^{|f||g|}(g f)^{\mathrm{op}}$. Finally, the curvature elements in $C^{\text {op }}$ are $h_{X^{\mathrm{op}}}=-h_{X}$. In particular, this defines the CDG-ring $B^{\mathrm{op}}=\left(B^{\mathrm{op}}, d^{\mathrm{op}}, h^{\mathrm{op}}\right)$ opposite to a CDG-ring $B=(B, d, h)$.

Now let $k$ be a commutative ring. A $k$-linear CDG-category is a CDG-category whose $\Gamma$-graded abelian groups of morphisms are endowed with $\Gamma$-graded $k$-module structures so that the compositions are $k$-bilinear and the differentials are $k$-linear. The tensor product $C \otimes_{k} D$ of two $k$-linear CDG-categories $C$ and $D$ is constructed as follows. The objects of $C \otimes_{k} D$ are pairs $\left(X^{\prime}, X^{\prime \prime}\right)$ of objects $X^{\prime} \in C$ and $X^{\prime \prime} \in D$. The $\Gamma$-graded $k$-module of morphisms $\operatorname{Hom}_{C \otimes_{k} D}\left(\left(X^{\prime}, X^{\prime \prime}\right),\left(Y^{\prime}, Y^{\prime \prime}\right)\right)$ is the tensor product $\operatorname{Hom}_{C}\left(X^{\prime}, Y^{\prime}\right) \otimes_{k} \operatorname{Hom}_{D}\left(X^{\prime \prime}, Y^{\prime \prime}\right)$; the differential $d$ on this module is defined by the formula $d\left(f^{\prime} \otimes f^{\prime \prime}\right)=d\left(f^{\prime}\right) \otimes f^{\prime \prime}+(-1)^{\left|f^{\prime}\right|} f^{\prime} \otimes d\left(f^{\prime \prime}\right)$. The curvature elements are $h_{\left(X^{\prime}, X^{\prime \prime}\right)}=h_{X^{\prime}} \otimes \operatorname{id}_{X^{\prime \prime}}+\mathrm{id}_{X^{\prime}} \otimes h_{X^{\prime \prime}}$.

1.3. QDG-functors. Let $C$ and $D$ be CDG-categories. A covariant $C D G$-functor $F: C \longrightarrow D$ is a homogeneous additive functor between the $\Gamma$-graded categories $C$ and $D$, endowed with fixed elements $a_{X} \in \operatorname{Hom}_{D}(F(X), F(X))$ of degree 1 for all objects $X \in C$ such that $F(d f)=d F(f)+a_{Y} F(f)-(-1)^{|f|} F(f) a_{X}$ for any morphism $f: X \longrightarrow Y$ in $C$ and $F\left(h_{X}\right)=h_{F(X)}+d a_{X}+a_{X}^{2}$ for any object $X$. A contravariant CDG-functor $C \longrightarrow D$ is defined as a covariant CDG-functor $C^{\text {op }} \longrightarrow D$ or, equivalently, a covariant CDG-functor $C \longrightarrow D^{\text {op }}$. The opposite CDG-functor $F^{\mathrm{op}}: C^{\mathrm{op}} \longrightarrow D^{\mathrm{op}}$ to a covariant CDG-functor $F: C \longrightarrow D$ is defined by the rule $(F, a)^{\mathrm{op}}=\left(F^{\mathrm{op}},-a\right)$.

(Covariant or contravariant) CDG-functors $C \longrightarrow D$ are objects of the $D G$ category of $C D G$-functors. The $\Gamma$-graded abelian group of morphisms between covariant CDG-functors $F$ and $G$ is the $\Gamma$-graded group of homogeneous morphisms, with the sign rule, between $F$ and $G$ considered as functors between $\Gamma$-graded categories. More precisely, a morphism $f: F \longrightarrow G$ of degree $n \in \Gamma$ is a collection of morphisms $f_{X}: F(X) \longrightarrow G(X)$ of degree $n$ in $D$ for all objects $X \in C$ such that $f_{Y} F(g)=(-1)^{n|g|} G(g) f_{X}$ for any morphism $g: X \longrightarrow Y$ in $C$. The differential $d$ on the $\Gamma$-graded group $\operatorname{Hom}(F, G)$ of morphisms between CDG-functors $F=(F, a)$ and $G=(G, b)$ is defined by the rule $(d f)_{X}=d\left(f_{X}\right)+b_{X} f_{X}-(-1)^{|f|} f_{X} a_{X}$.

A (covariant or contravariant) $Q D G$-functor $F$ between CDG-categories $C$ and $D$ is the same set of data as a CDG-functor satisfying the same equations, except for the equation connecting $F\left(h_{X}\right)$ with $h_{F(X)}$, which is omitted. QDG-functors $C \longrightarrow D$ are objects of the $C D G$-category of $Q D G$-functors. The $\Gamma$-graded abelian group of morphisms between QDG-functors and the differential on it are defined in exactly the same way as in the CDG-functor case. The curvature element of a QDG-functor $F: C \longrightarrow D$ is the endomorphism $h_{F}: F \longrightarrow F$ of degree 2 defined by the formula $\left(h_{F}\right)_{X}=h_{F(X)}+d a_{X}+a_{X}^{2}-F\left(h_{X}\right)$ for all $X \in C$.

The composition of QDG-functors $(F, a): C \longrightarrow D$ and $(G, b): D \longrightarrow E$ is the QDG-functor $(G \circ F, c)$, where $c_{X}=G\left(a_{X}\right)+b_{F(X)}$ for any object $X \in C$. A CDGfunctor or QDG-functor $F=(F, a): C \longrightarrow D$ is said to be strict if $a_{X}=0$ for all objects $X \in C$. The identity CDG-functor $\operatorname{Id}_{C}$ of a CDG-category $C$ is the strict 
CDG-functor $\left(\operatorname{Id}_{C}, 0\right)$. The composition of strict QDG-functors is a strict QDGfunctor, and the composition of (strict) CDG-functors is a (strict) CDG-functor.

Two CDG-functors $F: C \longrightarrow D$ and $G: D \longrightarrow C$ between CDG-categories $C$ and $D$ are called mutually inverse equivalences of CDG-categories if they are equivalences of the $\Gamma$-graded categories such that the adjunction isomorphisms $i: G F \longrightarrow \operatorname{Id}_{C}$ and $j: F G \longrightarrow \operatorname{Id}_{D}$ are closed morphisms of CDG-functors, i.e., $d(i)=0=d(j)$ (any one of the two equations implies the other one). A CDGfunctor $F: C \longrightarrow D$ is an equivalence if and only if it is fully faithful as a functor between $\Gamma$-graded categories and any object $Y \in D$ is a twist of an object $F(X)$ for some $X \in C$.

An equivalence $(F, G)$ between $C D G$-categories $C$ and $D$ is called a strict equivalence if the CDG-functors $F$ and $G$ are strict. A strict CDG-functor $F: C \longrightarrow D$ is a strict equivalence if and only if it is fully faithful as a functor between $\Gamma$-graded categories and any object $Y \in D$ is isomorphic to an object $F(X)$ for some $X \in C$.

A strict CDG-functor between DG-categories is called a DG-functor. An equivalence of DG-categories is their strict equivalence as CDG-categories.

If all objects of the category $D$ admit twists with all of their endomorphisms of degree 1 , then the embedding of the DG-category of strict CDG-functors $C \longrightarrow D$ into the DG-category of all CDG-functors is an equivalence of DG-categories, and the embedding of the CDG-category of strict QDG-functors $C \longrightarrow D$ into the CDG-category of all QDG-functors is a strict equivalence of CDG-categories.

A QDG-functor between $k$-linear CDG-categories is $k$-linear if its action on the $\Gamma$-graded $k$-modules of morphisms in the CDG-categories is $k$-linear. Given three $k$-linear CDG-categories $C, D, E$, the functor of composition of $k$-linear QDGfunctors $C \longrightarrow D$ and $D \longrightarrow E$ is a strict $k$-linear CDG-functor on the tensor product of the $k$-linear CDG-categories of QDG-functors. The composition (on either side) with a fixed CDG-functor is a strict CDG-functor between the CDGcategories of QDG-functors, and the composition with a fixed QDG-functor is a strict QDG-functor between such CDG-categories.

Given two $k$-linear QDG-functors $F^{\prime}=\left(F^{\prime}, a^{\prime}\right): C^{\prime} \longrightarrow D^{\prime}$ and $F^{\prime \prime}=\left(F^{\prime \prime}, a^{\prime \prime}\right): C^{\prime \prime}$ $\longrightarrow D^{\prime \prime}$, their tensor product $\left(F^{\prime} \otimes F^{\prime \prime}, a\right): C^{\prime} \otimes_{k} C^{\prime \prime} \longrightarrow D^{\prime} \otimes_{k} D^{\prime \prime}$ is defined by the rule $\left(F^{\prime} \otimes F^{\prime \prime}\right)\left(X^{\prime}, X^{\prime \prime}\right)=\left(F^{\prime}\left(X^{\prime}\right), F^{\prime \prime}\left(X^{\prime \prime}\right)\right)$ on the objects, $\left(F^{\prime} \otimes F^{\prime \prime}\right)\left(f^{\prime} \otimes f^{\prime \prime}\right)=$ $F^{\prime}\left(f^{\prime}\right) \otimes F^{\prime \prime}\left(f^{\prime \prime}\right)$ on the morphisms, and $a_{X^{\prime} \otimes X^{\prime \prime}}=a_{X^{\prime}} \otimes \mathrm{id}_{X^{\prime \prime}}+\mathrm{id}_{X^{\prime}} \otimes a_{X^{\prime \prime}}$. The tensor product of strict QDG-functors is a strict QDG-functor, and the tensor product of (strict) CDG-functors is a (strict) CDG-functor.

1.4. QDG-modules. A left $Q D G$-module over a small CDG-category $C$ is a strict covariant QDG-functor $C \longrightarrow \operatorname{Pre}(A b)$. Analogously, a right QDG-module over $C$ is a strict contravariant QDG-functor $C^{\text {op }} \longrightarrow \operatorname{Pre}(A b)$. (Left or right) CDG-modules over a CDG-category $C$ are similarly defined in terms of strict CDG-functors with values in the CDG-category $\operatorname{Pre}(A b)$. The CDG-categories of left and right QDGmodules over $C$ are denoted by $C$-mod ${ }^{\text {qdg }}$ and $\bmod ^{\text {qdg }}-C$; the DG-categories of left and right CDG-modules over $C$ are denoted by $C-\bmod ^{\text {cdg }}$ and $\bmod ^{\text {cdg }}-C$. Since the CDG-category $\operatorname{Pre}(A b)$ admits arbitrary twists, one obtains (strictly) equivalent (C)DG-categories by considering not necessarily strict QDG- or CDG-functors.

Given a CDG-ring or CDG-category $C$, we will denote by $C^{\#}$ the underlying $\Gamma$-graded ring or category. For a QDG-module $M$ over $C$, we similarly denote by $M^{\#}$ the underlying $\Gamma$-graded $C^{\#}$-module (i.e., the homogeneous additive functor from $C^{\#}$ to the $\Gamma$-graded category of $\Gamma$-graded abelian groups) of $M$. 
If $k$ is a commutative ring and $C$ is a $k$-linear CDG-category, then any QDGfunctor $C \longrightarrow \operatorname{Pre}(A b)$ can be lifted to a $k$-linear QDG-functor $C \longrightarrow \operatorname{Pre}(k$-mod $)$ in a unique way, where $k$-mod denotes the abelian category of $k$-modules. So the CDG-category $C$-mod ${ }^{\text {qdg }}$ can also be described as the CDG-category of (strict) $k$ linear QDG-functors $C \longrightarrow \operatorname{Pre}(k$-mod). Notice that another notation for the CDGcategory $\operatorname{Pre}(k$-mod $)$ is $k$-mod ${ }^{\text {qdg }}$, where $k$ is considered as a CDG-ring concentrated in degree 0 with the trivial differential and curvature, while $k$-mod ${ }^{\text {cdg }}$ is a notation for the DG-category of complexes of $k$-modules.

Let $C$ be a small $k$-linear CDG-category, $N$ be a right QDG-module over $C$, and $M$ be a left QDG-module. The tensor product $N^{\#} \otimes_{C} \# M^{\#}$ is a $\Gamma$-graded $k$-module defined as the quotient module of the direct sum of $N(X) \otimes_{k} M(X)$ over all objects $X \in C$ by the sum of the images of the maps $N(Y) \otimes_{k} M(X) \longrightarrow$ $N(X) \otimes_{k} M(X) \oplus N(Y) \otimes_{k} M(Y)$ over all homogeneous morphisms $X \longrightarrow Y$ in $C$. There is a natural differential on $N^{\#} \otimes_{C} M^{\#}$ defined by the usual formula $d(n \otimes m)=d(n) \otimes m+(-1)^{|n|} n \otimes d(m)$. The precomplex of $k$-modules so obtained is denoted by $N \otimes_{C} M$. The tensor product over $C$ is a strict CDG-functor

$$
\otimes_{C}: \bmod ^{\mathrm{qdg}}-C \times C-\bmod ^{\mathrm{qdg}} \longrightarrow k-\bmod ^{\mathrm{qdg}},
$$

and its restriction to the DG-subcategories of CDG-modules is a DG-functor

$$
\otimes_{C}: \bmod ^{\mathrm{cdg}} C \times C-\bmod ^{\mathrm{cdg}} \longrightarrow k-\bmod ^{\mathrm{cdg}} .
$$

A QDG-functor between CDG-categories $F: C \longrightarrow D$ induces a strict QDGfunctor of inverse image (restriction of scalars) $F^{*}: D$-mod ${ }^{\text {qdg }} \longrightarrow C$-mod ${ }^{\text {qdg }}$. Here we use the natural strict equivalence between the CDG-categories of arbitrary and strict QDG-functors $C \longrightarrow \operatorname{Pre}(A b)$. When $F$ is a CDG-functor, the functor $F^{*}$ is a strict CDG-functor, and it restricts to a DG-functor $D$-mod ${ }^{\text {cdg }} \longrightarrow C$ - mod $^{\text {cdg }}$. For any right QDG-module $N$ and left QDG-module $M$ over a $k$-linear CDG-category $D$ and a $k$-linear CDG-functor $F: C \longrightarrow D$ there is a natural map of precomplexes of $k$-modules $F^{*}(N) \otimes_{C} F^{*}(M) \longrightarrow N \otimes_{D} M$, commuting with the differentials.

For any CDG-category $B$ there is a natural strict CDG-functor $B \longrightarrow \bmod ^{\mathrm{qdg}}-B$ assigning to an object $X \in B$ the right QDG-module $R_{X}: Y \longmapsto \operatorname{Hom}_{B}(Y, X)$ over $B$. Here the differential on $R_{X}(Y)$ coincides with the differential on $\operatorname{Hom}_{B}(Y, X)$.

A CDG-module over a DG-category is called a DG-module. The DG-categories of left and right DG-modules over a small DG-category $C$ are denoted by $C$-mod ${ }^{\mathrm{dg}}$ and $\bmod ^{\mathrm{dg}}-C$. In particular, $k$-mod ${ }^{\mathrm{dg}}$ is yet another notation for the DG-category of complexes of $k$-modules for a commutative ring $k$. If $C$ is a $k$-linear DG-category, then the objects of $C$-mod ${ }^{\mathrm{dg}}$ can be viewed as DG-functors $C \longrightarrow k-$ mod $^{\mathrm{dg}}$ and the objects of $\bmod ^{\mathrm{dg}}-C$ can be viewed as DG-functors $C^{\text {op }} \longrightarrow k$-mod ${ }^{\mathrm{dg}}$.

Given left QDG-modules $M^{\prime}$ and $M^{\prime \prime}$ over $k$-linear CDG-categories $B^{\prime}$ and $B^{\prime \prime}$, their tensor product $M^{\prime} \otimes_{k} M^{\prime \prime}$ is the QDG-module over $B^{\prime} \otimes_{k} B^{\prime \prime}$ defined as the composition of the tensor product of strict QDG-functors $M^{\prime} \otimes M^{\prime \prime}: B^{\prime} \otimes_{k} B^{\prime \prime} \longrightarrow$ $\operatorname{Pre}(k$-mod $) \otimes_{k}$ Pre $(k$-mod $)$ with the strict CDG-functor of tensor product of precomplexes $\otimes_{k}$ : Pre $(k$-mod $) \otimes_{k} \operatorname{Pre}(k$-mod $) \longrightarrow \operatorname{Pre}(k$-mod $)$. The latter functor assigns to two precomplexes of $k$-modules their tensor product as $\Gamma$-graded $k$-modules, endowed with the differential defined by the usual formula. The tensor product of CDG-modules is a CDG-module. 
1.5. Pseudo-equivalences. Let us call a homogeneous additive functor $F^{\#}: C^{\#} \longrightarrow D^{\#}$ between $\Gamma$-graded additive categories $C^{\#}$ and $D^{\#}$ a pseudoequivalence if $F$ is fully faithful and any object $Y \in D^{\#}$ can be obtained from objects $F(X), \quad X \in C^{\#}$, using the operations of finite direct sum, shift, and passage to a direct summand.

A CDG-functor between CDG-categories $F: C \longrightarrow D$ is called a pseudo-equivalence if it is fully faithful as a functor between the $\Gamma$-graded categories and if any object $Y \in D$ can be obtained from objects $F(X), X \in C$, using the operations of finite direct sum, shift, twist, and passage to a direct summand.

The category of (left or right) $\Gamma$-graded modules over a small $\Gamma$-graded category $C^{\#}$ is abelian. Let us call a right $\Gamma$-graded module $N$ over $C^{\#}$ (finitely generated) free if it is a (finite) direct sum of representable modules $R_{X}$, where $X \in C^{\#}$. A $\Gamma$ graded module $P$ over $C^{\#}$ is a projective object in the abelian category of $\Gamma$-graded modules if and only if it is a direct summand of a free $\Gamma$-graded module. A $\Gamma$-graded module $P$ is a compact projective object (i.e., a projective object representing a covariant functor preserving infinite direct sums on the category of modules) if and only if it is a direct summand of a finitely generated free $\Gamma$-graded module. In this case, a $\Gamma$-graded module $P$ is said to be finitely generated projective.

Given a CDG-category $B$, denote by $\bmod _{\mathrm{fgp}}^{\mathrm{cdg}}-B$ and $\bmod _{\mathrm{fgp}}^{\mathrm{qdg}}-B$ the DG-category of right CDG-modules and the CDG-category of right QDG-modules over $B$, respectively, which are finitely generated projective as $\Gamma$-graded modules. The representable QDG-modules $R_{X}$ are obviously objects of $\bmod _{\mathrm{fgp}}^{\mathrm{qdg}}-B$, so there is a strict CDG-functor $R: B \longrightarrow \bmod _{\mathrm{fgp}}^{\mathrm{qdg}}-B$. There is also the strict CDG-functor of tautological embedding $I: \bmod _{\mathrm{fgp}}^{\mathrm{cdg}}-B \longrightarrow \bmod _{\mathrm{fgp}}^{\mathrm{qdg}}-B$.

Lemma A. The CDG-functors $R$ and $I$ are pseudo-equivalences.

Proof. First of all notice that any two objects of a CDG-category $C$ that are isomorphic in the $\Gamma$-graded category $C^{\#}$ are each other's twists. In particular, so are any two QDG-modules over a CDG-category $B$ that are isomorphic as $\Gamma$-graded $B^{\#}$-modules. Hence in order to prove that $R$ is a pseudo-equivalence, it suffices to show that any (finitely generated) projective $\Gamma$-graded right $B^{\#}$-module $P$ admits a QDG-module structure. Indeed, if there is a $\Gamma$-graded right $B^{\#}$-module $Q$ such that the $\Gamma$-graded module $P \oplus Q$ admits a differential $d$ making it a QDG-module and if $\iota: P \longrightarrow P \oplus Q$ and $\pi: P \oplus Q \longrightarrow P$ are the embedding of and the projection onto the direct summand $P$ in $P \oplus Q$, then the differential $\pi d \iota$ on $P$ makes it a QDG-module.

To prove that $I$ is a pseudo-equivalence, it suffices to show that the $\Gamma$-graded $B^{\#}$-module $P \oplus P[-1]$ admits a CDG-module structure for any (finitely generated) projective right $B^{\#}$-module $P$. Define the right CDG-module $Q$ over $B$ with the group $Q(X)$ consisting of formal expressions of the form $p^{\prime}+d\left(p^{\prime \prime}\right), \quad p^{\prime}, p^{\prime \prime} \in$ $P(X)$, with $P(X)$ embedded into $Q(X)$ as the set of all expressions $p+d(0)$. The differential $d$ on $Q(X)$, being restricted to $P(X)$, maps $p+d(0)$ to $0+d(p)$, and $B$ acts on $P \subset Q$ as it acts on $P$. The action of $B$ is extended from $P$ to $Q$ in a unique way making the Leibniz rule satisfied, and the differential $d$ is extended from $P$ to $Q$ in a unique way making the equation on $d^{2}$ hold (see [15, proof of Theorem 3.6] for explicit formulas). There is a natural exact sequence of $\Gamma$-graded $B^{\#}$-modules $0 \longrightarrow P^{\#} \longrightarrow Q^{\#} \longrightarrow P^{\#}[-1] \longrightarrow 0$, which splits, since $P^{\#}$ is projective. 
Lemma B. If $F: C \longrightarrow D$ is a pseudo-equivalence of small $C D G$-categories, then the induced strict $C D G$-functors $F^{*}: D$-mod ${ }^{\mathrm{qdg}} \longrightarrow C$-mod ${ }^{\mathrm{qdg}}$ and $\bmod ^{\mathrm{qdg}}-D \longrightarrow$ $\bmod ^{\mathrm{qdg}}-C$ are strict equivalences of $C D G$-categories. For any $Q D G$-modules $N \in$ $\bmod ^{\mathrm{qdg}}-D$ and $M \in D$-mod ${ }^{\mathrm{qdg}}$, the natural map $N \otimes_{D} M \longrightarrow F^{*}(N) \otimes_{C} F^{*}(M)$ is an isomorphism of precomplexes. Besides, the induced $D G$-functors $F^{*}: D$-mod ${ }^{\text {cdg }}$ $\longrightarrow C$-mod ${ }^{\mathrm{cdg}}$ and $\bmod ^{\mathrm{cdg}}-D \longrightarrow \bmod ^{\mathrm{cdg}}-C$ are equivalences of $D G$-categories.

Proof. First of all, it is obvious that if $F: C^{\#} \longrightarrow D^{\#}$ is a pseudo-equivalence of $\Gamma$-graded categories, then the induced functor of restriction of scalars between the categories of (left or right) $\Gamma$-graded modules over $D$ and $C$ is an equivalence of $\Gamma$-graded categories. These equivalences transform the functor of tensor product of $\Gamma$-graded modules over $D$ into the functor of tensor product of $\Gamma$-graded modules over $C$. Thus, it remains to check that any QDG-module over $C$ can be extended to a QDG-module over $D$. This is also straightforward.

More generally, one can see that the assertions of Lemma B hold for any CDGfunctor $F: C \longrightarrow D$ that is a pseudo-equivalence as a $\Gamma$-graded functor $C^{\#} \longrightarrow$ $D^{\#}$. The assertions of both Lemmas A and B remain valid if one replaces finitely generated projective modules with finitely generated free ones.

Lemma C. If a CDG-functor $F: C \longrightarrow D$ is a pseudo-equivalence of $C D G$ categories, then so is the $C D G$-functor $F^{\mathrm{op}}: C^{\mathrm{op}} \longrightarrow D^{\mathrm{op}}$. If k-linear $C D G$ functors $F^{\prime}: C^{\prime} \longrightarrow D^{\prime}$ and $F^{\prime \prime}: C^{\prime \prime} \longrightarrow D^{\prime \prime}$ are pseudo-equivalences of $C D G$ categories, then so is the CDG-functor $F^{\prime} \otimes F^{\prime \prime}: C^{\prime} \otimes_{k} C^{\prime \prime} \longrightarrow D^{\prime} \otimes_{k} D^{\prime \prime}$.

\section{EXT AND TOR OF THE SECOND KIND}

This section contains an exposition of the classical theory of the two kinds of differential derived functors, largely following [5], except that we deal with CDGcategories rather than DG-(co)algebras. The classical theory allows us to establish an isomorphism between the Hochschild (co)homology of the second kind of a CDGcategory $B$ and the DG-category $C$ of right CDG-modules over $B$ that are finitely generated and projective as graded $B$-modules. We also construct a natural map between the two kinds of Hochschild (co)homology of any DG-category $C$ linear over a field $k$.

2.1. Ext and Tor of the first kind. Given a DG-category $D$, denote by $Z^{0}(D)$ the category whose objects are the objects of $D$ and whose morphisms are the closed (i.e., annihilated by the differential) morphisms of degree 0 in $D$. Let $H^{0}(D)$ denote the category whose objects are the objects of $D$ and whose morphisms are the elements of the cohomology groups of degree 0 of the complexes of morphisms in $D$. The categories $Z^{0}(D)$ and $H^{0}(D)$ have preadditive category structures (i.e., the abelian group structures on the sets of morphisms). In addition, these categories are endowed with the shift functors $X \longmapsto X[n]$ for all $n \in \Gamma$, provided that shifts of all objects exist in $D$ (see Section 1.2). Finally, let $H(D)$ denote the $\Gamma$-graded category whose objects are the objects of $D$ and whose morphisms are the $\Gamma$-graded groups of cohomology of the complexes of morphisms in $D$.

Let $k$ be a commutative ring and $C$ be a small $k$-linear DG-category. Let us endow the additive categories $Z^{0}\left(C\right.$-mod $\left.{ }^{\mathrm{dg}}\right)$ and $Z^{0}\left(\bmod ^{\mathrm{dg}}-C\right)$ with the following exact category structures. A short sequence $M^{\prime} \longrightarrow M \longrightarrow M^{\prime \prime}$ of DG-modules and closed morphisms between them is exact if and only if both the short sequence 
of $\Gamma$-graded $C^{\#}$-modules $M^{\prime \#} \longrightarrow M^{\#} \longrightarrow M^{\prime \prime \#}$ and the short sequence of $\Gamma$ graded $H(C)$-modules of cohomology $H\left(M^{\prime}\right) \longrightarrow H(M) \longrightarrow H\left(M^{\prime \prime}\right)$ are exact in the abelian categories of $\Gamma$-graded modules and their homogeneous morphisms of degree 0 . In other words, for any object $X \in C$ the sequence $M^{\prime}(X) \longrightarrow M(X) \longrightarrow$ $M^{\prime \prime}(X)$ must be a short exact sequence of complexes of $k$-modules whose $\Gamma$-graded cohomology modules also form a short exact sequence (i.e., the boundary maps vanish).

Denote the additive category $Z^{0}\left(k\right.$-mod $\left.{ }^{\mathrm{dg}}\right)$ of $\Gamma$-graded complexes of $k$-modules with its exact category structure defined above by $\operatorname{Com}_{\mathrm{ex}}(k$-mod). Let $d$ denote the differentials on objects of $\mathrm{Com}_{\mathrm{ex}}(k$-mod). We will be interested in the derived categories $\mathrm{D}^{-}\left(\mathrm{Com}_{\mathrm{ex}}(k\right.$-mod $\left.)\right)$ and $\mathrm{D}^{+}\left(\mathrm{Com}_{\mathrm{ex}}(k\right.$-mod $\left.)\right)$ of complexes, bounded from above or below, over the exact category $\operatorname{Com}_{\mathrm{ex}}(k$-mod). The differential acting between the terms of a complex over $\operatorname{Com}_{\mathrm{ex}}(k$-mod) will be denoted by $\partial$.

The objects of $\mathrm{D}^{-}\left(\mathrm{Com}_{\mathrm{ex}}(k\right.$-mod $\left.)\right)$ can be viewed as bicomplexes with one grading by the integers bounded from above and the other grading by elements of the group $\Gamma$. (The differential $d$ preserves the grading by the integers, while changing the $\Gamma$-valued grading, and the differential $\partial$ raises the grading by the integers by 1 , while preserving the $\Gamma$-valued grading.) To any such bicomplex one can assign its $\Gamma$-graded total complex, constructed by taking infinite direct sums along the diagonals. This defines a triangulated functor from $\mathrm{D}^{-}\left(\mathrm{Com}_{\mathrm{ex}}(k\right.$-mod $\left.)\right)$ to the unbounded derived category of $\Gamma$-graded complexes of $k$-modules,

$$
\operatorname{Tot}^{\oplus}: \mathrm{D}^{-}\left(\mathrm{Com}_{\mathrm{ex}}(k \text {-mod })\right) \longrightarrow \mathrm{D}(k \text {-mod }) .
$$

Analogously, the objects of $\mathrm{D}^{+}\left(\mathrm{Com}_{\mathrm{ex}}(k\right.$-mod $\left.)\right)$ can be viewed as bicomplexes with one grading by the integers bounded from below and the other grading by elements of the group $\Gamma$. To any such bicomplex one can assign its $\Gamma$-graded total complex, constructed by taking infinite products along the diagonals. This defines a triangulated functor

$$
\operatorname{Tot}^{n}: \mathrm{D}^{+}\left(\mathrm{Com}_{\mathrm{ex}}(k-\bmod )\right) \longrightarrow \mathrm{D}(k-\bmod ) .
$$

Any complex over $\mathrm{Com}_{\mathrm{ex}}(k$-mod) bounded from above (resp., below) that becomes exact (with respect to the differential $\partial$ ) after passing to the cohomology of the $\Gamma$-graded complexes of $k$-modules (with respect to the differential $d$ ) is annihilated by the functor $\operatorname{Tot}^{\oplus}$ (resp., $\operatorname{Tot}^{\sqcap}$ ).

Remark. The latter assertion does not hold for the total complexes of unbounded complexes over $\mathrm{Com}_{\mathrm{ex}}(k$-mod), constructed by taking infinite direct sums or products along the diagonals. That is the reason why we define the functors $\operatorname{Tot}^{\oplus}$ and $\operatorname{Tot}^{\sqcap}$ for bounded complexes only. The assertion holds, however, for the functor of "Laurent totalization" of unbounded complexes, which coincides with $\operatorname{Tot}^{\oplus}$ for complexes bounded from above and with $\operatorname{Tot}^{\square}$ for complexes bounded from below. See [5] and the introduction to [15] (cf. Remark 2.2).

Now consider the functor of two arguments (see Section 1.4)

$$
\otimes_{C}: Z^{0}\left(\bmod ^{\mathrm{dg}}-C\right) \times Z^{0}\left(C-\bmod ^{\mathrm{dg}}\right) \longrightarrow \operatorname{Com}_{\mathrm{ex}}(k-\bmod ) .
$$

We would like to construct its left derived functor

$$
\otimes_{C}^{\mathbb{L}}: Z^{0}\left(\bmod ^{\mathrm{dg}}-C\right) \times Z^{0}\left(C-\bmod ^{\mathrm{dg}}\right) \longrightarrow \mathrm{D}^{-}\left(\operatorname{Com}_{\mathrm{ex}}(k-\bmod )\right) .
$$


For this purpose, notice that both exact categories $Z^{0}\left(\bmod ^{\mathrm{dg}}-C\right)$ and $Z^{0}\left(C\right.$-mod $\left.{ }^{\mathrm{dg}}\right)$ have enough projective objects. Specifically, for any object $X \in C$ the representable DG-module $R_{X} \in Z^{0}\left(\bmod ^{\mathrm{dg}}-C\right)$ is projective, and so is the cone of the identity endomorphism of $R_{X}$ (taken in the DG-category mod ${ }^{\mathrm{dg}}-C$ ). Any object of $Z^{0}\left(\bmod ^{\mathrm{dg}}-C\right)$ is the image of an admissible epimorphism acting from an (infinite) direct sum of shifts of objects of the above two types.

Given a right DG-module $N$ and a left DG-module $M$ over $C$, choose a left projective resolution $Q_{\bullet}$ of $N$ and a left projective resolution $P_{\bullet}$ of $M$ in the exact categories $Z^{0}\left(\bmod ^{\mathrm{dg}}-C\right)$ and $Z^{0}\left(C\right.$-mod $\left.{ }^{\mathrm{dg}}\right)$. When substituted as one of the arguments of the functor $\otimes_{C}$, any projective object of one of the exact categories of DG-modules makes this functor an exact functor from the other exact category of DG-modules to the exact category $\operatorname{Com}_{\text {ex }}(k$-mod). This allows us to define $N \otimes_{C}^{\mathbb{L}} M \in \mathrm{D}^{-}\left(\mathrm{Com}_{\text {ex }}(k\right.$-mod $\left.)\right)$ as the object represented either by the complex $Q_{\bullet} \otimes_{C} M$, or by the complex $N \otimes_{C} P \bullet$, or by the total complex of the bicomplex $Q_{\bullet} \otimes_{C} P_{\bullet}$.

Analogously, consider the functor of two arguments

$$
\operatorname{Hom}^{C}: Z^{0}\left(C-\bmod ^{\mathrm{dg}}\right)^{\mathrm{op}} \times Z^{0}\left(C-\bmod ^{\mathrm{dg}}\right) \longrightarrow \mathrm{Com}_{\mathrm{ex}}(k \text {-mod }),
$$

assigning to any two left DG-modules over $C$ the complex of morphisms between them as DG-functors $C \longrightarrow k$-mod ${ }^{\mathrm{dg}}$. We would like to construct its right derived functor

$$
\mathbb{R H o m}{ }^{C}: Z^{0}\left(C-\bmod ^{\mathrm{dg}}\right)^{\mathrm{op}} \times Z^{0}\left(C-\bmod ^{\mathrm{dg}}\right) \longrightarrow \mathrm{D}^{+}\left(\operatorname{Com}_{\mathrm{ex}}(k-\bmod )\right) .
$$

Notice that the exact category $Z^{0}\left(C\right.$-mod $\left.{ }^{\mathrm{dg}}\right)$ has enough injective objects. For any projective object $Q \in Z^{0}\left(\bmod ^{\mathrm{dg}}-C\right)$ and an injective $k$-module $I$, the object $\operatorname{Hom}_{k}(Q, I) \in Z^{0}\left(C\right.$-mod $\left.{ }^{\mathrm{dg}}\right)$ is injective, and any injective object in the exact category $Z^{0}\left(C-\bmod ^{\mathrm{dg}}\right)$ is a direct summand of an object of this type. To prove these assertions, it suffices to check that for any DG-module $M \in Z^{0}\left(\bmod ^{\mathrm{dg}}-C\right)$, any object $X \in C$, and any element of $M(X)$ or $H(M)(X)$ there is a DG-module $Q$ as above and a closed morphism of DG-modules $M \longrightarrow \operatorname{Hom}_{k}(Q, I)$ that is injective on the chosen element.

Given left DG-modules $L$ and $M$ over $C$, choose a left projective resolution $P \bullet$ of $L$ and a right injective resolution $J^{\bullet}$ of $M$ in the exact category $Z^{0}\left(C\right.$-mod $\left.{ }^{\mathrm{dg}}\right)$. Substituting a projective object as the first argument or an injective object as the second argument of the functor $\mathrm{Hom}^{C}$, one obtains an exact functor from the exact category of DG-modules in the other argument to the exact category $\mathrm{Com}_{\mathrm{ex}}(k$-mod $)$. This allows one to define $\mathbb{R} \operatorname{Hom}^{C}(L, M) \in \mathrm{D}^{+}\left(\mathrm{Com}_{\mathrm{ex}}(k\right.$-mod $\left.)\right)$ as the object represented either by the complex $\operatorname{Hom}^{C}\left(P_{\bullet}, M\right)$, or by the complex $\operatorname{Hom}^{C}\left(L, J^{\bullet}\right)$, or by the total complex of the bicomplex $\operatorname{Hom}^{C}\left(P_{\bullet}, J^{\bullet}\right)$.

Composing the derived functor $\otimes_{C}^{\mathbb{L}}$ with the functor $\operatorname{Tot}^{\oplus}$, we obtain the derived functor

$$
\text { Tor }{ }^{C}: Z^{0}\left(\bmod ^{\mathrm{dg}}-C\right) \times Z^{0}\left(C-\bmod ^{\mathrm{dg}}\right) \longrightarrow \mathrm{D}(k-\bmod ) .
$$

Similarly, composing the derived functor $\mathbb{R} \operatorname{Hom}^{C}$ with the functor $\operatorname{Tot}^{\square}$, we obtain the derived functor

$$
\operatorname{Ext}_{C}: Z^{0}\left(C-\bmod ^{\mathrm{dg}}\right)^{\mathrm{op}} \times Z^{0}\left(C-\bmod ^{\mathrm{dg}}\right) \longrightarrow \mathrm{D}(k-\bmod ) .
$$

One can compute the derived functors $\operatorname{Tor}^{C}$ and $\operatorname{Ext}_{C}$ using resolutions of a more general type than above. Specifically, let $N$ and $M$ be a left and a right 
DG-module over $C$. Let $\cdots \longrightarrow F_{2} \longrightarrow F_{1} \longrightarrow F_{0} \longrightarrow M$ be a complex of left DG-modules over $C$ and (closed morphisms between them) such that the complex of $\Gamma$-graded $H(C)$-modules

$$
\cdots \longrightarrow H\left(F_{2}\right) \longrightarrow H\left(F_{1}\right) \longrightarrow H\left(F_{0}\right) \longrightarrow H(M) \longrightarrow 0
$$

is exact. Assume that the DG-modules $F_{i}$ are $h$-flat (homotopy flat), i.e., for any $i \geqslant 0$ and any right DG-module $R$ over $C$ such that $H(R)=0$, one has $H\left(R \otimes_{C} F_{i}\right)=$ 0 . Let $Q_{\bullet}$ be a left projective resolution of the DG-module $N$ in the exact category of right DG-modules over $C$. Then the natural maps $\operatorname{Tot}^{\oplus}\left(Q_{\bullet} \otimes_{C} F_{\bullet}\right) \longrightarrow$ $\operatorname{Tot}^{\oplus}\left(Q_{\bullet} \otimes_{C} M\right)$ and $\operatorname{Tot}^{\oplus}\left(Q_{\bullet} \otimes_{C} F_{\bullet}\right) \longrightarrow \operatorname{Tot}^{\oplus}\left(N \otimes_{C} F_{\bullet}\right)$ are quasi-isomorphisms, so the $\Gamma$-graded complex of $k$-modules $\operatorname{Tot}^{\oplus}\left(N \otimes_{C} F_{\bullet}\right)$ represents the object $\operatorname{Tor}^{C}(N, M)$ in $\mathrm{D}(k$-mod $)$.

Analogously, let $L$ and $M$ be left DG-modules over $C$. Let $\cdots \longrightarrow P_{2} \longrightarrow$ $P_{1} \longrightarrow P_{0} \longrightarrow L$ be a complex of left DG-modules over $C$ which becomes exact after passing to the $\Gamma$-graded cohomology modules. Assume that the DG-modules $P_{i}$ are $h$-projective (homotopy projective), i.e., for any $i \geqslant 0$ and any left DG-module $R$ over $C$ such that $H(R)=0$, one has $H\left(\operatorname{Hom}^{C}\left(P_{i}, R\right)\right)=0$. Then the complex of $k$-modules $\operatorname{Tot}^{\sqcap}\left(\operatorname{Hom}^{C}\left(P_{\bullet}, M\right)\right)$ represents the object $\operatorname{Ext}_{C}(L, M)$ in $\mathrm{D}(k$-mod). Similarly, let $M \longrightarrow J^{0} \longrightarrow J^{1} \longrightarrow J^{2} \longrightarrow \cdots$ be a complex of left DG-modules over $C$ which becomes exact after passing to the cohomology modules. Assume that the DG-modules $J^{i}$ are $h$-injective, i.e., for any $i \geqslant 0$ and any left DG-module $R$ over $C$ such that $H(R)=0$, one has $H\left(\operatorname{Hom}^{C}\left(R, J^{i}\right)\right)=0$. Then the complex of $k$-modules $\operatorname{Tot}^{\sqcap}\left(\operatorname{Hom}^{C}\left(L, J^{\bullet}\right)\right)$ represents the object $\operatorname{Ext}_{C}(L, M)$.

In particular, it follows that the functors Tor ${ }^{C}$ and $\operatorname{Ext}_{C}$ transform quasiisomorphisms of DG-modules (i.e., morphisms of DG-modules inducing isomorphisms of the $\Gamma$-graded cohomology modules) in any of their arguments into isomorphisms in $\mathrm{D}(k$-mod).

Furthermore, consider the case when the complex of morphisms between any two objects of $C$ is an h-flat complex of $k$-modules. Then for any left DG-module $M$ over $C$ such that the complex of $k$-modules $M(X)$ is h-flat for any object $X \in C$, the bar-construction

$$
\begin{aligned}
\cdots \longrightarrow \bigoplus_{Y, Z \in C} C(X, Y) \otimes_{k} C(Y, Z) \otimes_{k} M(Z) & \\
& \longrightarrow \bigoplus_{Y \in C} C(X, Y) \otimes_{k} M(Y) \longrightarrow M(X),
\end{aligned}
$$

where we use the simplifying notation $C(X, Y)=\operatorname{Hom}_{C}(Y, X)$ for any objects $X$, $Y \in C$, defines a left resolution of the DG-module $M$ which consists of h-flat DGmodules over $C$ and remains exact after passing to the cohomology modules. Thus, for any right DG-module $N$ over $C$ the total complex of the bar-complex

$$
\cdots \longrightarrow \bigoplus_{Y, Z \in C} N(Y) \otimes_{k} C(Y, Z) \otimes_{k} M(Z) \longrightarrow \bigoplus_{X \in C} N(Y) \otimes_{k} M(Y),
$$

constructed by taking infinite direct sums along the diagonals, represents the object $\operatorname{Tor}^{C}(N, M)$ in $\mathrm{D}(k$-mod). The h-flatness condition on the DG-module $M$ can be replaced with a similar condition on the DG-module $N$.

Analogously, assume that the complex of morphisms between any two objects of $C$ is an h-projective complex of $k$-modules. Let $L$ and $M$ be left DG-modules over $C$ such that either the complex of $k$-modules $L(X)$ is h-projective for any object $X \in C$ or the complex of $k$-modules $M(X)$ is h-injective for any object $X \in C$. 
Then the total complex of the cobar-complex

$$
\begin{aligned}
\prod_{X \in C} \operatorname{Hom}_{k}(L(X), M(X)) & \longrightarrow \prod_{X, Y \in C} \operatorname{Hom}_{k}\left(C(X, Y) \otimes_{k} L(Y), M(X)\right) \\
& \cdots,
\end{aligned}
$$

constructed by taking infinite products along the diagonals, represents the object $\operatorname{Ext}_{C}(L, M)$ in $\mathrm{D}(k$-mod $)$.

Given a $k$-linear DG-functor $F: C \longrightarrow D$, a right DG-module $N$ over $D$, and a left DG-module $M$ over $D$, there is a natural morphism

$$
\operatorname{Tor}^{C}\left(F^{*} N, F^{*} M\right) \longrightarrow \operatorname{Tor}^{D}(N, M)
$$

in $\mathrm{D}(k$-mod $)$. Analogously, given a $k$-linear DG-functor $F: C \longrightarrow D$ and left DGmodules $L$ and $M$ over $D$, there is a natural morphism

$$
\operatorname{Ext}_{D}(L, M) \longrightarrow \operatorname{Ext}_{C}\left(F^{*} L, F^{*} M\right)
$$

in $\mathrm{D}(k$-mod). If the functor $H(F): H(C) \longrightarrow H(D)$ is a pseudo-equivalence of $\Gamma$-graded categories, then the natural morphisms between the objects Tor and Ext over $C$ and $D$ are isomorphisms for any DG-modules $L, M$, and $N$. This follows from the fact that the similar morphisms between the objects Tor and Ext over $H(C)$ and $H(D)$ are isomorphisms.

2.2. Ext and Tor of the second kind: General case. Let $B$ be a small $k$-linear CDG-category. Then the categories $Z^{0}\left(B-\bmod ^{\text {cdg }}\right)$ and $Z^{0}\left(\bmod ^{\text {cdg }}-B\right)$ of (left and right) CDG-modules over $B$ and closed morphisms of degree 0 between them are abelian. In particular, consider the abelian category $Z^{0}\left(k\right.$-mod $\left.{ }^{\text {cdg }}\right)$ of $\Gamma$-graded complexes of $k$-modules and denote it by $\operatorname{Com}_{\mathrm{ab}}(k$-mod). We will be interested in the derived categories $\mathrm{D}^{-}\left(\mathrm{Com}_{\mathrm{ab}}(k\right.$-mod $\left.)\right)$ and $\mathrm{D}^{+}\left(\mathrm{Com}_{\mathrm{ab}}(k\right.$-mod $\left.)\right)$ of complexes, bounded from above or below, over the abelian category $\operatorname{Com}_{\mathrm{ab}}(k$-mod).

The objects of $\mathrm{D}^{-}\left(\mathrm{Com}_{\mathrm{ab}}(k\right.$-mod $\left.)\right)$ can be viewed as bicomplexes with one grading by the integers bounded from above and the other grading by elements of the group $\Gamma$. To any such bicomplex one can assign its $\Gamma$-graded total complex, constructed by taking infinite products along the diagonals. This defines a triangulated functor

$$
\operatorname{Tot}^{\sqcap}: \mathrm{D}^{-}\left(\mathrm{Com}_{\mathrm{ab}}(k-\bmod )\right) \longrightarrow \mathrm{D}(k \text {-mod }) \text {. }
$$

Analogously, the objects of $\mathrm{D}^{+}\left(\mathrm{Com}_{\mathrm{ab}}(k\right.$-mod $\left.)\right)$ can be viewed as bicomplexes with one grading by the integers bounded from below and the other grading by elements of the group $\Gamma$. To any such bicomplex one can assign its $\Gamma$-graded total complex, constructed by taking infinite direct sums along the diagonals. This defines a triangulated functor

$$
\operatorname{Tot}^{\oplus}: \mathrm{D}^{+}\left(\mathrm{Com}_{\mathrm{ab}}(k-\bmod )\right)-\longrightarrow \mathrm{D}(k-\bmod ) .
$$

Remark. The functors of total complexes of unbounded complexes over $\mathrm{Com}_{\mathrm{ab}}(k$-mod $)$, constructed by taking infinite direct sums or infinite products along the diagonals, are not well-defined on the derived category $\mathrm{D}\left(\mathrm{Com}_{\mathrm{ab}}(k\right.$-mod $\left.)\right)$. The procedure of "Laurent totalization" of unbounded complexes, which coincides with $\operatorname{Tot}^{\sqcap}$ for complexes bounded from above and with $\operatorname{Tot}^{\oplus}$ for complexes bounded from below, defines a functor on $\mathrm{D}\left(\mathrm{Com}_{\mathrm{ab}}(k\right.$-mod $\left.)\right)$, though. Notice that this Laurent totalization is different from the one discussed in Remark 2.1 (the chosen direction along the diagonals is opposite in the two cases). 
Now consider the functor of two arguments (see Section 1.4)

$$
\otimes_{B}: Z^{0}\left(\bmod ^{\mathrm{cdg}}-B\right) \times Z^{0}\left(B-\bmod ^{\mathrm{cdg}}\right) \longrightarrow \operatorname{Com}_{\mathrm{ab}}(k-\mathrm{mod}) .
$$

We would like to construct its left derived functor

$$
\otimes_{B}^{\mathbb{L}}: Z^{0}\left(\bmod ^{\mathrm{cdg}}-B\right) \times Z^{0}\left(B-\bmod ^{\mathrm{cdg}}\right) \longrightarrow \mathrm{D}^{-}\left(\operatorname{Com}_{\mathrm{ab}}(k-\bmod )\right) .
$$

Notice that the abelian categories $Z^{0}\left(\bmod ^{\mathrm{cdg}}-B\right)$ and $Z^{0}\left(B-\bmod ^{\mathrm{cdg}}\right)$ have enough projective objects. More precisely, for any projective left $\Gamma$-graded module $P$ over $B^{\#}$ the corresponding freely generated CDG-module $Q$, as constructed in the proof of Lemma 1.5. A, is a projective object of $Z^{0}\left(B-\bmod ^{\mathrm{cdg}}\right)$. Any projective object in $Z^{0}\left(B\right.$-mod $\left.{ }^{\text {cdg }}\right)$ is a direct summand of an object of this type. For any projective object $Q$ in $Z^{0}\left(B\right.$-mod $\left.{ }^{\text {cdg }}\right)$, the underlying left $\Gamma$-graded $B^{\#}$-module $Q^{\#}$ is projective.

Let us call a left $\Gamma$-graded $B^{\#}$-module $P^{\#}$ flat if the functor of tensor product with $P^{\#}$ over $B^{\#}$ is exact on the abelian category of right $\Gamma$-graded $B^{\#}$ modules. Given a left CDG-module $P$ over $B$, if the left $B^{\#}$-module $P^{\#}$ is flat, then the functor of tensor product with $P$ is exact as a functor $Z^{0}\left(\bmod ^{\text {cdg }}-B\right) \longrightarrow$ $\mathrm{Com}_{\mathrm{ab}}\left(k\right.$-mod). Any projective $\Gamma$-graded $B^{\#}$-module is flat.

Given a right CDG-module $N$ and a left CDG-module $M$ over $B$, choose a left resolution $Q \bullet$ of $N$ in $Z^{0}\left(\bmod ^{\text {cdg }}-B\right)$ and a left resolution $P_{\bullet}$ of $M$ in $Z^{0}\left(B\right.$-mod $\left.{ }^{\text {cdg }}\right)$ such that the $\Gamma$-graded $B^{\#}$-modules $Q_{i}^{\#}$ and $P_{i}^{\#}$ are flat. In view of the above remarks, we can define $N \otimes_{B}^{\mathbb{L}} M \in \mathrm{D}^{-}\left(\mathrm{Com}_{\mathrm{ab}}(k\right.$-mod $\left.)\right)$ as the object represented either by the complex $Q_{\bullet} \otimes_{B} M$, or by the complex $N \otimes_{B} P_{\bullet}$, or by the total complex of the bicomplex $Q_{\bullet} \otimes_{B} P_{\bullet}$.

Analogously, consider the functor of two arguments

$$
\operatorname{Hom}^{B}: Z^{0}\left(B-\bmod ^{\text {cdg }}\right)^{\text {op }} \times Z^{0}\left(B-\bmod ^{\text {cdg }}\right) \longrightarrow \operatorname{Com}_{\mathrm{ab}}(k \text {-mod }),
$$

assigning to any two left CDG-modules over $B$ the complex of morphisms between them as strict $C D G$-functors $B \longrightarrow k$ - od $^{\text {cdg }}$. We would like to construct its right derived functor

$$
\mathbb{R H o m}^{B}: Z^{0}\left(B-\bmod ^{\text {cdg }}\right)^{\text {op }} \times Z^{0}\left(B-\bmod ^{\text {cdg }}\right) \longrightarrow \mathrm{D}^{+}\left(\operatorname{Com}_{\mathrm{ab}}(k-\bmod )\right) .
$$

Notice that the abelian category $Z^{0}\left(B\right.$-mod $\left.{ }^{\text {cdg }}\right)$ has enough injective objects. For any injective object $J$ in $Z^{0}\left(B\right.$-mod $\left.{ }^{\text {cdg }}\right)$, the underlying left $\Gamma$-graded $B^{\#}$-module $J^{\#}$ is injective. One can construct these injective CDG-modules as the duals to projective (or flat) right CDG-modules (see the discussion of injective DG-modules in Section 2.1) or obtain them as the CDG-modules cofreely cogenerated by injective $\Gamma$-graded $B^{\#}$-modules (see the construction of injective resolutions in [15, proof of Theorem 3.6]).

Given left CDG-modules $L$ and $M$ over $B$, choose a left resolution $P \bullet$ of $L$ and a right resolution $J^{\bullet}$ of $M$ in $Z^{0}\left(B\right.$-mod $\left.{ }^{\text {cdg }}\right)$ such that the $\Gamma$-graded $B^{\#}$ modules $P_{i}^{\#}$ are projective and the $\Gamma$-graded $B^{\#}$-modules $J^{i \#}$ are injective. Define $\mathbb{R H o m}^{B}(L, M) \in \mathrm{D}^{-}\left(\mathrm{Com}_{\mathrm{ab}}(k\right.$-mod $\left.)\right)$ as the object represented either by the complex $\operatorname{Hom}^{B}\left(P_{\bullet}, M\right)$, or by the complex $\operatorname{Hom}^{B}\left(L, J^{\bullet}\right)$, or by the total complex of the bicomplex $\operatorname{Hom}^{B}\left(P_{\bullet}, J^{\bullet}\right)$.

Composing the derived functor $\otimes_{B}^{\mathbb{L}}$ with the functor Tot ${ }^{\sqcap}$, we obtain the derived functor

$$
\operatorname{Tor}^{B, I I}: Z^{0}\left(\bmod ^{\mathrm{cdg}}-B\right) \times Z^{0}\left(B-\bmod ^{\mathrm{cdg}}\right) \longrightarrow \mathrm{D}(k-\bmod ) .
$$


Similarly, composing the derived functor $\mathbb{R H o m}^{B}$ with the functor $\operatorname{Tot}^{\oplus}$, we obtain the derived functor

$$
\operatorname{Ext}_{B}^{I I}: Z^{0}\left(B-\bmod ^{\text {cdg }}\right)^{\text {op }} \times Z^{0}\left(B-\bmod ^{\text {cdg }}\right) \longrightarrow \mathrm{D}(k \text {-mod }) .
$$

The derived functors $\operatorname{Tor}^{B, I I}$ and $\operatorname{Ext}_{B}^{I I}$ are called the Tor and Ext of the second kind of CDG-modules over $B$.

Notice that the derived functors $\otimes_{B}^{\mathbb{L}}$ and $\mathbb{R} \operatorname{Hom}^{B}$ assign distinguished triangles to short exact sequences of CDG-modules in any argument, hence so do the derived functors $\operatorname{Tor}^{B, I I}$ and $\operatorname{Ext}_{B}^{I I}$.

Given a $k$-linear CDG-functor $F: B \longrightarrow C$, a right CDG-module $N$ over $C$, and a left CDG-module $M$ over $C$, there is a natural morphism

$$
\operatorname{Tor}^{B, I I}\left(F^{*} N, F^{*} M\right) \longrightarrow \operatorname{Tor}^{C, I I}(N, M)
$$

in $\mathrm{D}(k$-mod). Analogously, given a $k$-linear CDG-functor $F: B \longrightarrow C$ and left CDG-modules $L$ and $M$ over $C$, there is a natural morphism

$$
\operatorname{Ext}_{C}^{I I}(L, M) \longrightarrow \operatorname{Ext}_{B}^{I I}\left(F^{*} L, F^{*} M\right)
$$

in $\mathrm{D}(k$-mod $)$. If the functor $F^{\#}: B^{\#} \longrightarrow C^{\#}$ is a pseudo-equivalence of $\Gamma$-graded categories, then these natural morphisms are isomorphisms for any CDG-modules $L, M$, and $N$.

Now let $C$ be a small $k$-linear DG-category. Then the identity functors from the exact categories $Z^{0}\left(\bmod ^{\mathrm{dg}}-C\right)$ and $Z^{0}\left(C-\bmod ^{\mathrm{dg}}\right)$ to the abelian categories $Z^{0}\left(\bmod ^{\mathrm{cdg}}-C\right)$ and $Z^{0}\left(C-\bmod ^{\mathrm{cdg}}\right)$ are exact, so any resolution in $Z^{0}\left(\bmod ^{\mathrm{dg}}-C\right)$ or $Z^{0}\left(C-\bmod ^{\mathrm{dg}}\right)$ is also a resolution in $Z^{0}\left(\bmod ^{\mathrm{cdg}}-C\right)$ or $Z^{0}\left(C-\bmod ^{\mathrm{cdg}}\right)$. Besides, any DG-module that is projective or injective in the exact category $Z^{0}\left(\bmod ^{\mathrm{dg}}-C\right)$ or $Z^{0}\left(C\right.$-mod $\left.{ }^{\mathrm{dg}}\right)$ is also projective or injective as a $\Gamma$-graded $C^{\#}$-module. It follows that there are natural morphisms

$$
\operatorname{Tor}^{C}(N, M) \longrightarrow \operatorname{Tor}^{C, I I}(N, M)
$$

and

$$
\operatorname{Ext}_{C}^{I I}(L, M) \longrightarrow \operatorname{Ext}_{C}(L, M)
$$

in $\mathrm{D}(k$-mod $)$ for any DG-modules $L, M$, and $N$ over $C$.

2.3. Flat/projective case. Let $B$ be a small $k$-linear CDG-category, $N$ a right CDG-module over $B$, and $M$ a left CDG-module over $B$. Consider the $\Gamma$-graded complex of $k$-modules $\operatorname{Bar}^{\sqcap}(N, B, M)$ constructed in the following way. As a $\Gamma$ graded $k$-module, $\operatorname{Bar}^{\sqcap}(N, B, M)$ is obtained by totalizing a bigraded $k$-module with one grading by elements of the group $\Gamma$ and the other grading by nonpositive integers, the totalizing being performed by taking infinite products along the diagonals. The component of degree $-i \in \mathbb{Z}$ of that bigraded module is the $\Gamma$-graded $k$-module

$$
\bigoplus_{X_{0}, \ldots, X_{i} \in B} N\left(X_{0}\right) \otimes_{k} B\left(X_{0}, X_{1}\right) \otimes_{k} \cdots \otimes_{k} B\left(X_{i-1}, X_{i}\right) \otimes_{k} M\left(X_{i}\right),
$$

where, as in Section 2.1, we use the simplifying notation $B(X, Y)=\operatorname{Hom}_{B}(Y, X)$.

The differential on $\operatorname{Bar}^{\sqcap}(N, B, M)$ is the sum of the three components $\partial, d$, and $\delta$ given by the formulas

$$
\begin{aligned}
& \partial\left(n \otimes b_{1} \otimes \cdots \otimes b_{i} \otimes m\right)=n b_{1} \otimes b_{2} \otimes \cdots \otimes b_{i} \otimes m-n \otimes b_{1} b_{2} \otimes b_{3} \otimes \cdots \otimes b_{i} \otimes m \\
& +\cdots+(-1)^{i-1} n \otimes b_{1} \otimes \cdots \otimes b_{i-2} \otimes b_{i-1} b_{i} \otimes m+(-1)^{i} n \otimes b_{1} \otimes \cdots \otimes b_{i-1} \otimes b_{i} m,
\end{aligned}
$$


where the products $b_{j} b_{j+1}$ denote the composition of morphisms in $B$ and the products $n b_{1}$ and $b_{i} m$ denote the action of morphisms in $B$ on the CDG-modules,

$$
\begin{aligned}
(-1)^{i} d\left(n \otimes b_{1} \otimes \cdots \otimes b_{i} \otimes m\right) & =d(n) \otimes b_{1} \otimes \cdots \otimes b_{i} \otimes m \\
& +(-1)^{|n|} n \otimes d\left(b_{1}\right) \otimes b_{2} \otimes \cdots \otimes b_{i} \otimes m+\cdots \\
& +(-1)^{|n|+\left|b_{1}\right|+\cdots+\left|b_{i}\right|} n \otimes b_{1} \otimes \cdots \otimes b_{i} \otimes d(m)
\end{aligned}
$$

and

$$
\begin{aligned}
& \delta\left(n \otimes b_{1} \otimes \cdots \otimes b_{i} \otimes m\right)=n \otimes h \otimes b_{1} \otimes \cdots \otimes b_{i} \otimes m \\
& \quad-n \otimes b_{1} \otimes h \otimes b_{2} \otimes \cdots \otimes b_{i} \otimes m+\cdots+(-1)^{i} n \otimes b_{1} \otimes \cdots \otimes b_{i} \otimes h \otimes m .
\end{aligned}
$$

Proposition A. Assume that all the $\Gamma$-graded $k$-modules $B^{\#}(X, Y)$ are flat, and either all the $\Gamma$-graded $k$-modules $N^{\#}(X)$ are flat or all the $\Gamma$-graded $k$-modules $M^{\#}(X)$ are flat. Then the complex $\operatorname{Bar}^{\sqcap}(N, B, M)$ represents the object $\operatorname{Tor}^{B, I I}(N, M)$ in the derived category $\mathrm{D}(k$-mod $)$.

Proof. Choose a left resolution $Q_{\bullet}$ of the right CDG-module $N$ and a left resolution $P_{\bullet}$ of the left CDG-module $M$ such that the $\Gamma$-graded $B^{\#}$-modules $P_{j}^{\#}$ and $Q_{j}^{\#}$ are flat. Consider the tricomplex $\operatorname{Bar}^{\sqcap}\left(Q_{\bullet}, B, P_{\bullet}\right)$ and construct its $\Gamma$-graded total complex by taking infinite products along the diagonals. Then this total complex maps naturally to both the complex $\operatorname{Bar}^{\sqcap}(N, B, M)$ and the total complex $\operatorname{Tot}^{\sqcap}\left(Q_{\bullet} \otimes_{B} P_{\bullet}\right)$ of the tricomplex $Q_{\bullet} \otimes_{B} P_{\bullet}$, also constructed by taking infinite products along the diagonals. These morphisms of $\Gamma$-graded complexes are both quasi-isomorphisms (cf. the proof of Proposition 2.4. A below, where some additional details can be found).

Let $F: B \longrightarrow C$ be a $k$-linear CDG-functor, $N$ be a right CDG-module over $C$, and $M$ be a left CDG-module over $C$. Then there is a natural morphism of complexes of $k$-modules $F_{*}: \operatorname{Bar}^{\sqcap}\left(F^{*} N, B, F^{*} M\right) \longrightarrow \operatorname{Bar}^{\sqcap}(N, C, M)$ given by the rule

$$
\begin{aligned}
F_{*}\left(n \otimes b_{1} \otimes \cdots \otimes b_{i} \otimes m\right) & =\sum_{j_{0}, \ldots, j_{i}=0}^{\infty}(-1)^{\rho\left(j_{0}, \ldots, j_{i} ;|n|,\left|b_{1}\right|, \ldots,\left|b_{i}\right|\right)} \\
n & \otimes a^{\otimes j_{0}} \otimes F\left(b_{1}\right) \otimes a^{\otimes j_{1}} \otimes \cdots \otimes F\left(b_{i}\right) \otimes a^{\otimes j_{i}} \otimes m,
\end{aligned}
$$

where

$$
\begin{aligned}
& \rho\left(j_{0}, \ldots, j_{i} ; t_{0}, t_{1}, \ldots, t_{i}\right)=\left(j_{0}+\cdots+j_{i}-1\right)\left(j_{0}+\cdots+j_{i}\right) / 2 \\
& +j_{0}(i+1)+j_{1} i+\cdots+j_{i}+j_{0} t_{0}+j_{1}\left(t_{0}+t_{1}\right)+\cdots+j_{i}\left(t_{0}+t_{1}+\cdots+t_{i}\right) .
\end{aligned}
$$

The image of an arbitrary element in $\operatorname{Bar}^{\sqcap}\left(F^{*} N, B, F^{*} M\right)$ is constructed as the sum of the images of (the infinite number of) its bihomogeneous components, the sum being convergent bidegree-wise in $\operatorname{Bar}^{\sqcap}(N, C, M)$.

Suppose the CDG-categories $B$ and $C$ satisfy the assumptions of Proposition A, and so does one of the CDG-modules $N$ and $M$. Then the morphism of barcomplexes $F_{*}$ represents the morphism (7) of the objects Tor in $\mathrm{D}(k$-mod).

Now let $L$ and $M$ be left CDG-modules over $B$. Consider the $\Gamma$-graded complex of $k$-modules $\mathrm{Cob}^{\oplus}(L, B, M)$ constructed as follows. As a $\Gamma$-graded $k$-module, $\operatorname{Cob}^{\oplus}(L, B, M)$ is obtained by totalizing a bigraded $k$-module with one grading by elements of the group $\Gamma$ and the other grading by nonnegative integers, the totalizing 
being done by taking infinite direct sums along the diagonals. The component of degree $i \in \mathbb{Z}$ of that bigraded module is the $\Gamma$-graded $k$-module

$$
\prod_{X_{0}, \ldots, X_{i} \in B} \operatorname{Hom}_{k}\left(B\left(X_{0}, X_{1}\right) \otimes_{k} \cdots \otimes_{k} B\left(X_{i-1}, X_{i}\right) \otimes_{k} L\left(X_{i}\right), M\left(X_{0}\right)\right) .
$$

The differential on $\mathrm{Cob}^{\oplus}(L, B, M)$ is the sum of the three components $\partial, d$, and $\delta$ given by the formulas

$$
\begin{aligned}
& (\partial f)\left(b_{1}, \ldots, b_{i+1}, l\right)=(-1)^{|f|\left|b_{1}\right|} b_{1} f\left(b_{2}, \ldots, b_{i-1}, l\right)-f\left(b_{1} b_{2}, b_{3}, \ldots, b_{i+1}, l\right) \\
& \quad+\cdots+(-1)^{i} f\left(b_{1}, \ldots, b_{i-1}, b_{i} b_{i+1}, l\right)+(-1)^{i+1} f\left(b_{1}, \ldots, b_{i}, b_{i+1} l\right), \\
& (-1)^{i}(d f)\left(b_{1}, \ldots, b_{i}, l\right)=d\left(f\left(b_{1}, \ldots, b_{i}, l\right)\right)-(-1)^{|f|} f\left(d b_{1}, b_{2}, \ldots, b_{i}, l\right) \\
& -(-1)^{|f|+\left|b_{1}\right|} f\left(b_{1}, d b_{2}, b_{3}, \ldots, b_{i}, l\right)-\cdots-(-1)^{|f|+\left|b_{1}\right|+\ldots+\left|b_{i}\right|} f\left(b_{1}, \ldots, b_{i}, d l\right),
\end{aligned}
$$

and

$$
\begin{aligned}
(\delta f)\left(b_{1}, \ldots, b_{i-1}, l\right) & =-f\left(h, b_{1}, \ldots, b_{i-1}, l\right) \\
& +f\left(b_{1}, h, \ldots, b_{i-1}, l\right)-\cdots+(-1)^{i} f\left(b_{1}, \ldots, b_{i-1}, h, l\right) .
\end{aligned}
$$

Proposition B. Assume that all the $\Gamma$-graded $k$-modules $B^{\#}(X, Y)$ are projective, and either all the $\Gamma$-graded $k$-modules $L^{\#}(X)$ are projective or all the $\Gamma$-graded $k$ modules $M^{\#}(X)$ are injective. Then the complex $\operatorname{Cob}^{\oplus}(L, B, M)$ represents the object $\operatorname{Ext}_{B}^{I I}(L, M)$ in the derived category $\mathrm{D}(k$-mod $)$.

Proof. Choose a left resolution $P_{\bullet}$ of the left CDG-module $L$ and a right resolution $J^{\bullet}$ of the left CDG-module $M$ such that the $\Gamma$-graded $B^{\#}$-modules $P_{j}^{\#}$ are projective and the $\Gamma$-graded $B^{\#}$-modules $Q^{j \#}$ are injective. Consider the tricomplex $\mathrm{Cob}^{\oplus}\left(P_{\bullet}, B, J^{\bullet}\right)$ and construct its $\Gamma$-graded total complex by taking infinite direct sums along the diagonals. Both the complex $\operatorname{Cob}^{\oplus}(L, B, M)$ and the total complex $\operatorname{Tot}^{\oplus}\left(\operatorname{Hom}^{B}\left(P_{\bullet}, J^{\bullet}\right)\right)$ of the tricomplex $\operatorname{Hom}^{B}\left(P_{\bullet}, J^{\bullet}\right)$ map quasi-isomorphically into the above total complex.

Let $F: B \longrightarrow C$ be a $k$-linear CDG-functor, and $L$ and $M$ be left CDGmodules over $C$. Then there is a natural morphism of complexes of $k$-modules $F^{*}: \operatorname{Cob}^{\mathrm{op}}(L, C, M) \longrightarrow \operatorname{Cob}^{\mathrm{op}}\left(F^{*} L, B, F^{*} M\right)$ given by the rule

$$
\begin{aligned}
\left(F^{*} f\right)\left(b_{1} \otimes \cdots \otimes b_{i} \otimes l\right)= & \sum_{j_{0}, \ldots, j_{i}=0}^{\infty}(-1)^{\lambda\left(j_{0}, \ldots, j_{i} ;|f|,\left|b_{1}\right|, \ldots,\left|b_{i}\right|\right)} \\
& f\left(a^{\otimes j_{0}} \otimes F\left(b_{1}\right) \otimes a^{\otimes j_{1}} \otimes \cdots \otimes F\left(b_{i}\right) \otimes a^{j_{i}} \otimes l\right),
\end{aligned}
$$

where

$$
\begin{aligned}
\lambda\left(j_{0}, \ldots, j_{i} ; t_{0}, t_{1}, \ldots, t_{i}\right) & =j_{0} i+j_{1}(i-1)+\cdots+j_{i-1} \\
& +j_{0} t_{0}+j_{1}\left(t_{0}+t_{1}\right)+\cdots+j_{i}\left(t_{0}+t_{1}+\cdots+t_{i}\right) .
\end{aligned}
$$

Suppose the CDG-categories $B$ and $C$ satisfy the assumptions of Proposition B, and so does one of the CDG-modules $L$ and $M$. Then the morphism of cobarcomplexes $F^{*}$ represents the morphism (8) of the objects Ext in $\mathrm{D}(k$-mod).

Denote by $\operatorname{Bar}^{\oplus}(N, B, M)$ the $\Gamma$-graded complex of $k$-modules constructed in the same way as $\operatorname{Bar}^{\sqcap}(N, B, M)$, except that the totalization is being done by taking infinite direct sums along the diagonals. Similarly, denote by $\operatorname{Cob}^{\sqcap}(L, B, M)$ the $\Gamma$-graded complex of $k$-modules constructed in the same way as $\operatorname{Cob}^{\oplus}(L, B, M)$ except that the totalization is being done by taking infinite products along the diagonals. 
Assume that $C$ is a small DG-category in which the complex of morphisms between any two objects is an h-flat complex of flat $k$-modules, and either a right DG-module $N$ or a left DG-module $M$ over $C$ is such that all the complexes of $k$ modules $N(X)$ or $M(X)$ are h-flat complexes of flat $k$-modules. Then the natural map $\operatorname{Bar}^{\oplus}(N, C, M) \longrightarrow \operatorname{Bar}^{\sqcap}(N, C, M)$ represents the morphism $\operatorname{Tor}^{C}(N, M) \longrightarrow$ $\operatorname{Tor}^{C, I I}(N, M)$ in $\mathrm{D}(k$-mod).

Analogously, assume that the complex of morphisms between any two objects in a DG-category $C$ is an h-projective complex of projective $k$-modules, and either a left DG-module $L$ over $C$ is such that all the complexes of $k$-modules $L(X)$ are hprojective complexes of projective $k$-modules or a left DG-module $M$ over $C$ is such that all the complexes of $k$-modules $M(X)$ are h-injective complexes of injective $k$-modules. Then the natural map $\operatorname{Cob}^{\oplus}(L, C, M) \longrightarrow \operatorname{Cob}^{\sqcap}(L, C, M)$ represents the morphism $\operatorname{Ext}_{C}^{I I}(L, M) \longrightarrow \operatorname{Ext}_{C}(L, M)$ in $\mathrm{D}(k$-mod $)$.

Notice that the complexes $\operatorname{Bar}^{\oplus}(N, B, M)$ and $\operatorname{Cob}^{\sqcap}(L, B, M)$ are not functorial with respect to nonstrict CDG-functors between CDG-categories $B$ because of the infinite summation in the formulas (11) and (13).

Proposition C. Let $B$ be a small k-linear CDG-category. Assume that the maps $k \longrightarrow \operatorname{Hom}_{B}(X, X)$ corresponding to the curvature elements $h_{X} \in \operatorname{Hom}_{B}(X, X)$ admit $k$-linear retractions $\operatorname{Hom}_{B}(X, X) \longrightarrow k$, i.e., they are embeddings of $k$ module direct summands. In particular, this holds when $k$ is a field and all the elements $h_{X}$ are nonzero. Then for any $C D G$-modules $L, M$ and $N$ the complexes $\operatorname{Bar}^{\oplus}(N, B, M)$ and $\operatorname{Cob}^{\sqcap}(L, B, M)$ are acyclic.

Proof. This follows from the fact that the differentials $\delta$ on the bigraded bar- and cobar-complexes are acyclic.

2.4. Hochschild (co)homology. Let $B$ be a small $k$-linear CDG-category. Consider the CDG-category $B \otimes_{k} B^{\text {op }}$. Since it is naturally isomorphic to its opposite CDG-category, there is no need to distinguish between the left and the right CDGmodules over it. Furthermore, there is a natural (left) CDG-module over the CDGcategory $B \otimes_{k} B^{\text {op }}$ assigning to an object $\left(X, Y^{\mathrm{op}}\right) \in B \otimes_{k} B^{\text {op }}$ the precomplex of $k$-modules $B(X, Y)=\operatorname{Hom}_{B}(Y, X)$. By an abuse of notation, we will denote this CDG-module (as well as the corresponding right CDG-module) simply by $B$. Assume that the $\Gamma$-graded $k$-modules $B^{\#}(X, Y)$ are flat for all objects $X, Y \in B$.

The Hochschild homology of the second kind $H H_{*}^{I I}(B, M)$ of a $k$-linear CDGcategory $B$ with coefficients in a (left) CDG-module $M$ over $B \otimes_{k} B^{\text {op }}$ is defined as the homology of the object $\operatorname{Tor}^{B \otimes_{k} B^{\mathrm{op}}, I I}(B, M) \in \mathrm{D}(k$-mod). In particular, the Hochschild homology of the second kind of the CDG-module $M=B$ over $B \otimes_{k} B^{\text {op }}$ is simply called the Hochschild homology of the second kind of the $k$-linear CDGcategory $B$ and denoted by $H H_{*}^{I I}(B, B)=H H_{*}^{I I}(B)$.

The Hochschild cohomology of the second kind $H H^{I I}, *(B, M)$ of a $k$-linear CDGcategory $B$ with coefficients in a (left) CDG-module $M$ over $B \otimes_{k} B^{\text {op }}$ is defined as the cohomology of the object $\operatorname{Ext}_{B \otimes_{k} B^{\circ \mathrm{p}}}^{I I}(B, M) \in \mathrm{D}(k$-mod $)$. In particular, the Hochschild cohomology of the second kind of the CDG-module $M=B$ over $B \otimes_{k} B^{\mathrm{op}}$ is simply called the Hochschild cohomology of the second kind of the $k$-linear CDG-category $B$ and denoted by $H H^{I I, *}(B, B)=H H^{I I}, *(B)$.

Remark. We define the Hochschild (co)homology of the second kind for CDG-categories $B$ satisfying the above flatness assumption only, even though our definition 
makes sense without this requirement. In fact, this assumption is never used in this paper (except in the discussion of explicit complexes further in this section, which requires a stronger projectivity assumption in the cohomology case anyway). However, we believe that our definition is not the right one without the flatness assumption, since one is not supposed to use underived nonexact functors when defining (co)homology theories. So to define the Hochschild (co)homology of the second kind in the general case, one would need to replace a CDG-category $B$ with a CDG-category, equivalent to it in some sense and satisfying the flatness requirement. We do not know how such a replacement would look. The analogue of this procedure for Hochschild (co)homology of the first kind is well known (in this case it suffices to replace a DG-category $C$ with a quasi-equivalent DG-category with h-flat complexes of morphisms; see below).

By the result of Section 2.3, the Hochschild homology $H H_{*}^{I I}(B, M)$ is computed by the explicit bar-complex $\operatorname{Bar}^{\sqcap}\left(B, B \otimes_{k} B^{\mathrm{op}}, M\right)$. When the $\Gamma$-graded $k$-modules $B^{\#}(X, Y)$ are projective for all objects $X, Y \in B$, the Hochschild cohomology $H H^{I I}, *(B, M)$ is computed by the explicit cobar-complex $\operatorname{Cob}^{\oplus}\left(B, B \otimes_{k} B^{\mathrm{op}}, M\right)$. However, these complexes are too big and apparently not very useful.

There are smaller and much more important complexes computing the Hochschild (co)homology, namely, the Hochschild complexes. The homological Hochschild complex of the second kind $\operatorname{Hoch}_{\bullet}(B, M)$ is constructed in the following way. As a $\Gamma$-graded $k$-module, $\operatorname{Hoch}_{\bullet}^{\Pi}(B, M)$ is obtained by taking infinite products along the diagonals of a bigraded $k$-module with one grading by elements of the group $\Gamma$ and the other grading by nonpositive integers. The component of degree $-i \in \mathbb{Z}$ of that bigraded $k$-module is the $\Gamma$-graded $k$-module

$$
\bigoplus_{X_{0}, \ldots, X_{i} \in B} M\left(X_{i}, X_{0}^{\mathrm{op}}\right) \otimes_{k} B\left(X_{0}, X_{1}\right) \otimes_{k} \cdots \otimes_{k} B\left(X_{i-1}, X_{i}\right) .
$$

The differential on $\operatorname{Hoch}_{\bullet}^{\square}(B, M)$ is the sum of the three components $\partial, d$, and $\delta$ given by the formulas

$$
\begin{aligned}
\partial\left(m \otimes b_{1} \otimes \cdots \otimes b_{i}\right) & =m b_{1} \otimes b_{2} \otimes \cdots \otimes b_{i}-m \otimes b_{1} b_{2} \otimes b_{3} \otimes \cdots \otimes b_{i} \\
& +\cdots+(-1)^{i-1} m \otimes b_{1} \otimes \cdots \otimes b_{i-2} \otimes b_{i-1} b_{i} \\
& +(-1)^{i+\left|b_{i}\right|\left(|m|+\left|b_{1}\right|+\cdots+\left|b_{i-1}\right|\right)} b_{i} m \otimes b_{1} \otimes \cdots \otimes b_{i-1}, \\
(-1)^{i} d\left(m \otimes b_{1} \otimes \cdots \otimes b_{i}\right) & =d(m) \otimes b_{1} \otimes \cdots \otimes b_{i}+(-1)^{|m|} m \otimes d\left(b_{1}\right) \otimes b_{2} \otimes \cdots \otimes b_{i} \\
& +\cdots+(-1)^{|m|+\left|b_{1}\right|+\cdots+\left|b_{i-1}\right|} m \otimes b_{1} \otimes \cdots \otimes b_{i-1} \otimes d\left(b_{i}\right),
\end{aligned}
$$

and

$$
\begin{aligned}
\delta\left(m \otimes b_{1} \otimes \cdots \otimes b_{i}\right) & =m \otimes h \otimes b_{1} \otimes \cdots \otimes b_{i} \\
& \quad-m \otimes b_{1} \otimes h \otimes b_{2} \otimes \cdots \otimes b_{i}+\cdots+(-1)^{i} m \otimes b_{1} \otimes \cdots \otimes b_{i} \otimes h .
\end{aligned}
$$

Proposition A. The homology of the complex $\operatorname{Hoch}_{\bullet}(B, M)$ is naturally isomorphic to the Hochschild homology of the second kind $H H_{*}^{I I}(B, M)$ as a $\Gamma$-graded $k$-module.

Proof. Choose a left resolution $P_{\bullet}$ of the CDG-module $M$ such that the $\Gamma$-graded $B^{\#} \otimes_{k} B^{\# \text { op }}$-modules $P_{j}^{\#}$ are flat. Consider the $\operatorname{bicomplex} \operatorname{Hoch}_{\bullet}\left(B, P_{\bullet}\right)$ and construct its total complex by taking infinite products along the diagonals. This total complex maps naturally to both the complex $\operatorname{Hoch}_{\bullet}^{\Pi}(B, M)$ and the total complex 
of the bicomplex $B \otimes_{B \otimes_{k} B^{\text {op }}} P_{\bullet}$, constructed by taking infinite products along the diagonals. These morphisms of $\Gamma$-graded complexes are both quasi-isomorphisms.

Indeed, the morphism $\operatorname{Hoch}_{\bullet}^{\Pi}\left(B, P_{\bullet}\right) \longrightarrow \operatorname{Hoch}_{\bullet}^{\Pi}(B, M)$ is a quasi-isomorphism, because the functor $\operatorname{Hoch}_{\bullet}^{\Pi}(B,-)$ transforms exact sequences of CDG-modules over $B \otimes_{k} B^{\text {op }}$ into exact sequences of complexes. The morphism $\operatorname{Hoch}_{\bullet}\left(B, P_{\bullet}\right) \longrightarrow$ $B \otimes_{B \otimes_{k} B^{\text {op }}} P_{\bullet}$ is a quasi-isomorphism, since the morphism $\operatorname{Hoch}_{\bullet}(B, P) \longrightarrow P$ is a quasi-isomorphism for any CDG-module $P$ over $B \otimes_{k} B^{\text {op }}$ such that the $\Gamma$-graded $B^{\#} \otimes_{k} B^{\# \text { op }}$-module $P^{\#}$ is flat. The latter assertion follows from a similar statement for the bigraded Hochschild complex of the $\Gamma$-graded $B^{\#} \otimes_{k} B^{\# \text { op }}$-module $P^{\#}$ with the differential $\partial$.

Let $F: B \longrightarrow C$ be a $k$-linear CDG-functor and $M$ be a CDG-module over $C \otimes_{k}$ $C^{\text {op }}$. Let us denote the CDG-module $\left(F \otimes F^{\text {op }}\right)^{*} M$ over $B \otimes_{k} B^{\text {op }} \operatorname{simply}$ by $F^{*} M$. There is a natural morphism of complexes of $k$-modules $F_{*}: \operatorname{Hoch}_{\bullet}\left(B, F^{*} M\right) \longrightarrow$ $\operatorname{Hoch}_{\bullet}(C, M)$ defined by the rule

$$
\begin{aligned}
F_{*}\left(m \otimes b_{1} \otimes \cdots \otimes b_{i}\right)=\sum_{j_{0}, \ldots, j_{i}=0}^{\infty}(-1)^{\rho\left(j_{0}, \ldots, j_{i} ;|m|,\left|b_{1}\right|, \ldots,\left|b_{i}\right|\right)} & \\
& m \otimes a^{\otimes j_{0}} \otimes F\left(b_{1}\right) \otimes a^{\otimes j_{1}} \otimes \cdots \otimes F\left(b_{i}\right) \otimes a^{\otimes j_{i}},
\end{aligned}
$$

where the value of $\rho$ in the exponent is given by formula (12). The image of an arbitrary element in $\operatorname{Hoch}_{\bullet}^{\Pi}\left(B, F^{*} M\right)$ is constructed as the sum of the images of (the infinite number of) its bihomogeneous components, the sum being convergent bidegree-wise in $\operatorname{Hoch}^{\bullet}(C, M)$.

The morphism $F_{*}$ of Hochschild complexes computes the map of Hochschild homology

$$
H H_{*}^{I I}\left(B, F^{*} M\right) \longrightarrow H H_{*}^{I I}(C, M)
$$

obtained by passing to the homology in the morphism of Tor objects (7) for the CDG-functor $F \otimes F^{\mathrm{op}}$. Furthermore, there is a natural closed morphism $B \longrightarrow F^{*} C$ of CDG-modules over $B \otimes_{k} B^{\text {op }}$ inducing a map of Hochschild homology

$$
H H_{*}^{I I}(B) \longrightarrow H H_{*}^{I I}(C)
$$

and a morphism of Hochschild complexes $F_{*}$ : $\operatorname{Hoch}_{\bullet}^{\square}(B, B) \longrightarrow \operatorname{Hoch}_{\bullet}^{\sqcap}(C, C)$ computing this homology map.

The cohomological Hochschild complex of the second kind $\operatorname{Hoch}^{\oplus, \bullet}(B, M)$ is constructed as follows. As a $\Gamma$-graded $k$-module, $\operatorname{Hoch}_{\bullet}(B, M)$ is obtained by taking infinite direct sums along the diagonals of a bigraded $k$-module with one grading by elements of the group $\Gamma$ and the other grading by nonnegative integers. The component of degree $i \in \mathbb{Z}$ of that bigraded $k$-module is the $\Gamma$-graded $k$-module

$$
\prod_{X_{0}, \ldots, X_{i} \in B} \operatorname{Hom}_{k}\left(B\left(X_{0}, X_{1}\right) \otimes_{k} \cdots \otimes_{k} B\left(X_{i-1}, X_{i}\right), M\left(X_{0}, X_{i}^{\mathrm{op}}\right)\right) .
$$

The differential on $\operatorname{Hoch}^{\oplus, \bullet}(B, M)$ is the sum of the three components $\partial, d$, and $\delta$ given by the formulas

$$
\begin{aligned}
&(\partial f)\left(b_{1}, \ldots, b_{i+1}\right)=(-1)^{|f|\left|b_{1}\right|} b_{1} f\left(b_{2}, \ldots, b_{i+1}\right)-f\left(b_{1} b_{2}, b_{3}, \ldots, b_{i+1}\right) \\
&+\cdots+(-1)^{i} f\left(b_{1}, \ldots, b_{i-1}, b_{i} b_{i+1}\right)+(-1)^{i+1} f\left(b_{1}, \ldots, b_{i}\right) b_{i+1} \\
&(-1)^{i}(d f)\left(b_{1}, \ldots, b_{i}\right)=d\left(f\left(b_{1}, \ldots, b_{i}\right)\right)-(-1)^{|f|} f\left(d b_{1}, b_{2}, \ldots, b_{i}\right) \\
&-\cdots-(-1)^{|f|+\left|b_{1}\right|+\cdots+\left|b_{i-1}\right|} f\left(b_{1}, \ldots, b_{i-1}, d b_{i}\right)
\end{aligned}
$$


and

$$
\begin{aligned}
(\delta f)\left(b_{1}, \ldots, b_{i-1}\right) & =-f\left(h, b_{1}, \ldots, b_{i-1}\right) \\
& +f\left(b_{1}, h, b_{2}, \ldots, b_{i-1}\right)-\cdots+(-1)^{i} f\left(b_{1}, \ldots, b_{i-1}, h\right) .
\end{aligned}
$$

Proposition B. Assume that all the $\Gamma$-graded $k$-modules $B^{\#}(X, Y)$ are projective. Then the cohomology of the complex $\operatorname{Hoch}^{\oplus, \bullet}(B, M)$ is naturally isomorphic to the Hochschild cohomology of the second kind $H^{I I, *}(B, M)$ as a $\Gamma$-graded $k$-module.

Proof. Choose a right resolution $J^{\bullet}$ of the CDG-module $M$ such that the $\Gamma$-graded $B^{\#} \otimes_{k} B^{\# \text { op }}$-modules $J^{j \#}$ are injective. Consider the bicomplex $\operatorname{Hoch}^{\oplus, \bullet}\left(B, J^{\bullet}\right)$ and construct its total complex by taking infinite direct sums along the diagonals. Both the complex $\operatorname{Hoch}^{\oplus, \bullet}(B, M)$ and the total complex of the bicomplex $\operatorname{Hom}^{B \otimes_{k} B^{\text {op }}}\left(B, J^{\bullet}\right)$ map quasi-isomorphically into the above total complex.

For a $k$-linear CDG-functor $F: B \longrightarrow C$ and a CDG-module $M$ over $C \otimes_{k} C^{\text {op }}$, there is a natural morphism of complexes of $k$-modules $F^{*}: \operatorname{Hoch}^{\oplus, \bullet}(C, M) \longrightarrow$ $\operatorname{Hoch}^{\oplus, \bullet}\left(B, F^{*} M\right)$ defined by the rule

$$
\begin{aligned}
\left(F^{*} f\right)\left(b_{1} \otimes \cdots \otimes b_{i}\right)=\sum_{j_{0}, \ldots, j_{i}=0}^{\infty}(-1)^{\lambda\left(j_{0}, \ldots, j_{i} ;|f|,\left|b_{1}\right|, \ldots,\left|b_{i}\right|\right)} & \\
& \quad f\left(a^{\otimes j_{0}} \otimes F\left(b_{1}\right) \otimes a^{\otimes j_{1}} \otimes \cdots \otimes F\left(b_{i}\right) \otimes a^{j_{i}}\right),
\end{aligned}
$$

where the value of $\lambda$ in the exponent is given by formula (14).

Suppose the CDG-categories $B$ and $C$ satisfy the assumptions of Proposition B. Then the morphism $F^{*}$ of Hochschild complexes computes the map of Hochschild cohomology

$$
H H^{I I, *}(C, M) \longrightarrow H H^{I I, *}\left(B, F^{*} M\right)
$$

obtained by passing to the cohomology in the morphism of Ext objects (8) for the CDG-functor $F \otimes F^{\mathrm{op}}$. Notice that, unlike the Hochschild homology, the Hochschild cohomology of CDG-categories $H H^{I I}, *(B)$ is not functorial with respect to arbitrary CDG-functors $F: B \longrightarrow C$. It is contravariantly functorial, however, with respect to CDG-functors $F$ for which the functor $F^{\#}: B^{\#} \longrightarrow C^{\#}$ is fully faithful, since the closed morphism of CDG-modules $B \longrightarrow F^{*} C$ is an isomorphism in this case.

The (co)homology of the complexes $\operatorname{Hoch}_{\bullet}^{\sqcap}(B, M)$ and $\operatorname{Hoch}^{\oplus, \bullet}(B, M)$ are what is called the "Borel-Moore Hochschild homology" and the "compactly supported Hochschild cohomology" in [1].

Now denote by $\operatorname{Hoch}_{\bullet}^{\oplus}(B, M)$ the $\Gamma$-graded complex of $k$-modules constructed in the same way as $\operatorname{Hoch}_{\bullet}(B, M)$, except that the totalization is being done by taking infinite direct sums along the diagonals. Similarly, denote by $\operatorname{Hoch}^{\sqcap, \bullet}(B, M)$ the $\Gamma$-graded complex of $k$-modules constructed in the same way as $\operatorname{Hoch}^{\oplus, \bullet}(B, M)$, except that the totalization is being done by taking infinite products. The complexes $\operatorname{Hoch}_{\bullet}^{\oplus}(B, M)$ and $\operatorname{Hoch}^{\sqcap, \bullet}(B, M)$ play an important role when $B$ is a DG-category, but apparently not otherwise, as we will see below.

Let $C$ be a small $k$-linear DG-category. Assume that the complexes of $k$-modules $C(X, Y)$ are h-flat for all objects $X, Y \in C$. The (conventional) Hochschild homology (of the first kind) $H H_{*}(C, M)$ of a $k$-linear DG-category $C$ with coefficients in 
a DG-module $M$ over $C \otimes_{k} C^{\text {op }}$ is the homology of the object Tor ${ }^{C \otimes_{k} C^{\text {op }}}(C, M) \in$ $\mathrm{D}(k$-mod $)$. In particular, the Hochschild homology of the DG-module $M=C$ over $C$ is simply called the Hochschild homology of $C$ and denoted by $H_{*}(C, C)=$ $H H_{*}(C)$.

The (conventional) Hochschild cohomology (of the first kind) $H H^{*}(C, M)$ of a $k$-linear DG-category $C$ with coefficients in a DG-module $M$ over $C \otimes_{k} C^{\text {op }}$ is the cohomology of the object $\operatorname{Ext}_{C \otimes_{k} C^{\text {op }}}(C, M) \in \mathrm{D}(k$-mod $)$. In particular, the Hochschild cohomology of the DG-module $M=C$ over $C$ is simply called the Hochschild cohomology of $C$ and denoted by $H H^{*}(C, C)=H H^{*}(C)$.

Let $F: C \longrightarrow D$ be a $k$-linear DG-functor between DG-categories whose complexes of morphisms are h-flat complexes of $k$-modules. Then for any DG-module $M$ over $D \otimes_{k} D^{\text {op }}$ passing to the homology in the morphism of Tor objects (3) for the DG-functor $F \otimes F^{\mathrm{op}}$ provides a natural map of $\Gamma$-graded $k$-modules

$$
H H_{*}\left(C, F^{*} M\right) \longrightarrow H H_{*}(D, M) .
$$

Composing this map with the map induced by the closed morphism $C \longrightarrow F^{*} D$ of DG-modules over $C \otimes_{k} C^{\text {op }}$, we obtain a natural map

$$
H H_{*}(C) \longrightarrow H H_{*}(D) \text {. }
$$

Passing to the cohomology in the morphism of Ext objects (4) for the DG-functor $F \otimes F^{\mathrm{op}}$ provides a natural map

$$
H H^{*}(D, M) \longrightarrow H H^{*}\left(C, F^{*} M\right) .
$$

Unlike the Hochschild homology, the Hochschild cohomology of DG-categories $H H^{*}(C)$ is not functorial with respect to arbitrary DG-functors $F: C \longrightarrow D$. It is contravariantly functorial, however, with respect to DG-functors $F$ such that the functor $H(F): H(C) \longrightarrow H(D)$ is fully faithful, since the closed morphism of DG-modules $C \longrightarrow F^{*} D$ is a quasi-isomorphism in this case.

When the functor $H(F)$ is a pseudo-equivalence of $\Gamma$-graded categories, the maps (20) - (22) are isomorphisms, as is the natural map $H H^{*}(D) \longrightarrow H H^{*}(C)$. Indeed, under our assumptions on the DG-categories $C$ and $D$ the $\Gamma$-graded category $H\left(C \otimes_{k} C^{\text {op }}\right)$ is isomorphic to $H(C) \otimes_{k} H(C)^{\text {op }}$ and similarly for $D$, so the assertion follows from Lemma 1.5. C and the results of Section 2.1.

Just as in Section 2.1] one shows that the complex $\operatorname{Hoch}_{\bullet}^{\oplus}(C, M)$ computes the Hochschild homology $H H_{*}(C, M)$. The morphism of complexes $F_{*}$ : $\operatorname{Hoch}_{\bullet}^{\oplus}\left(C, F^{*} M\right)$ $\longrightarrow \operatorname{Hoch}_{\bullet}^{\oplus}(D, M)$ induced by a DG-functor $F$ computes the map of the Hochschild homology (20). In particular, the morphism of complexes $F_{*}$ : $\operatorname{Hoch}_{\bullet}^{\oplus}(C, C)$ $\longrightarrow \operatorname{Hoch}_{\bullet}^{\oplus}(D, D)$ induced by $F$ computes the map (21). When all the complexes of morphisms in $C$ are h-projective complexes of $k$-modules, the complex $\operatorname{Hoch}^{\sqcap, \bullet}(C, M)$ computes the Hochschild cohomology $H H^{*}(C, M)$. When both DG-categories $C$ and $D$ satisfy the same condition, the morphism of complexes $F^{*}: \operatorname{Hoch}^{\sqcap, \bullet}(D, M) \longrightarrow \operatorname{Hoch}^{\Pi, \bullet}\left(C, F^{*} M\right)$ computes the map (22).

When the complexes $C(X, Y)$ are h-flat complexes of flat $k$-modules for all objects $X, Y \in C$, both the Hochschild (co)homology of the first and the second kind are defined for any DG-module $M$ over $C \otimes_{k} C^{\text {op }}$. In this case, there are natural morphisms of $\Gamma$-graded $k$-modules

$$
H H_{*}(C, M) \longrightarrow H H_{*}^{I I}(C, M) \text { and } H H^{I I, *}(C, M) \longrightarrow H H^{*}(C, M),
$$


and, in particular,

$$
H H_{*}(C) \longrightarrow H H_{*}^{I I}(C) \text { and } H H^{I I, *}(C) \longrightarrow H H^{*}(C) .
$$

All of these are obtained from the comparison morphisms (9)-(10) for the two kinds of functors Tor and Ext. The morphism $H H_{*}(C, M) \longrightarrow H H_{*}^{I I}(C, M)$ is computed by the morphism of complexes $\operatorname{Hoch}_{\bullet}^{\oplus}(C, M) \longrightarrow \operatorname{Hoch}_{\bullet}^{\sqcap}(C, M)$. When the complexes $C(X, Y)$ are h-projective complexes of projective $k$-modules for all objects $X, Y \in C$, the morphism $H H^{I I, *}(C, M) \longrightarrow H H^{*}(C, M)$ is computed by the morphism of complexes $\operatorname{Hoch}^{\oplus, \bullet}(C, M) \longrightarrow \operatorname{Hoch}^{\sqcap, \bullet}(C, M)$.

On the other hand, assume that the maps $k \longrightarrow \operatorname{Hom}_{B}(X, X)$ corresponding to the curvature elements $h_{X} \in \operatorname{Hom}_{B}(X, X)$ are embeddings of $k$-linear direct summads. Then for any CDG-module $M$ over $B \otimes_{k} B^{\text {op }}$ the $\operatorname{complexes}^{\operatorname{Hoch}_{\bullet}^{\oplus}}(B, M)$ and $\operatorname{Hoch}^{\sqcap, \bullet}(B, M)$ are acyclic [1, Lemma 3.9 and Theorem 4.2(a)]. Notice that these complexes are not functorial with respect to nonstrict CDG-functors between CDG-categories $B$ because of the infinite summation in the formulas (15) and (18). The complexes $\operatorname{Hoch}_{\bullet}^{\oplus}(B, M)$ and $\operatorname{Bar}^{\oplus}\left(B, B \otimes_{k} B^{\text {op }}, M\right)$ are not quasi-isomorphic in general, even when $k$ is a field, $B$ is a CDG-algebra considered as a CDG-category

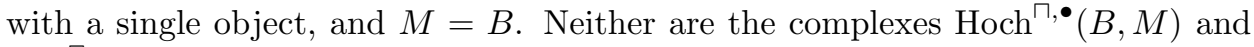
$\operatorname{Cob}^{\sqcap}\left(B, B \otimes_{k} B^{\text {op }}, M\right)$.

2.5. Change of the grading group. Let us first introduce some terminology that will be used throughout the rest of the paper. A $\Gamma$-graded module $N^{\#}$ over a $\Gamma$-graded category $B^{\#}$ is said to have flat dimension $d$ if $d$ is the minimal length of a left flat resolution of $N^{\#}$ in the abelian category of $\Gamma$-graded $B^{\#}$-modules or, equivalently, the functor of tensor product with $N^{\#}$ over $B^{\#}$ has the homological dimension $d$. Projective and injective dimensions of $\Gamma$-graded $B^{\#}$-modules are defined in a similar way. The left homological dimension of a $\Gamma$-graded category $B^{\#}$ is the homological dimension of the abelian category of $\Gamma$-graded left $B^{\#}$ modules, and the weak homological dimension of $B^{\#}$ is the homological dimension of the functor of tensor product of $\Gamma$-graded modules over $B^{\#}$.

Let $(\Gamma, \sigma, \mathbf{1})$ and $\left(\Gamma^{\prime}, \sigma^{\prime}, \mathbf{1}^{\prime}\right)$ be two different grading group data (see Section 1.1) and $\phi: \Gamma \longrightarrow \Gamma^{\prime}$ be a morphism of abelian groups taking $\mathbf{1}$ to $\mathbf{1}^{\prime}$ such that $\sigma$ is the pull-back of $\sigma^{\prime}$ by $\phi$. Then to any $\Gamma^{\prime}$-graded $k$-module $V^{\prime}$ one can assign a $\Gamma$-graded $k$-module $\phi^{*} V^{\prime}$ defined by the rule $\left(\phi^{*} V\right)^{n}=V^{\prime \phi(n)}$ for $n \in \Gamma$.

The functor $\phi^{*}$ has a left adjoint functor $\phi_{\text {! }}$ and a right adjoint functor $\phi_{*}$. The former assigns to a $\Gamma$-graded $k$-module $V$ the $\Gamma^{\prime}$-graded $k$-module $V^{\prime}$ constructed by taking the direct sums of the grading components of $V$ over all the preimages in $\Gamma$ of a given element $n^{\prime} \in \Gamma^{\prime}$, while the latter involves taking direct products over the preimages of $n^{\prime}$ in $\Gamma$.

All three functors $\phi_{!}, \phi_{*}$, and $\phi^{*}$ are exact. Besides, they transform (pre)complexes of $k$-modules to (pre)complexes of $k$-modules and commute with passing to the cohomology of the complexes of $k$-modules. So they induce triangulated functors between the derived categories $\mathrm{D}_{\Gamma}(k$-mod $)$ and $\mathrm{D}_{\Gamma^{\prime}}(k$-mod $)$ of $\Gamma$-graded and $\Gamma^{\prime}$-graded complexes of $k$-modules.

Given a $\Gamma$-graded $k$-linear CDG-category $B$, one can apply the functor $\phi_{\text {! to all }}$ its precomplexes of morphisms, obtaining a $\Gamma^{\prime}$-graded $k$-linear CDG-category $\phi_{!} B$. To a (left or right) CDG-module $M^{\prime}$ over $\phi_{!} B$ one can assign a CDG-module $\phi^{*} M^{\prime}$ over $B$, and to a CDG-module $M$ over $B$ one can assign CDG-modules $\phi_{!} M$ and $\phi_{*} M$ over $\phi_{!} B$. 
The functors $\phi_{!}, \phi_{*}$, and $\phi^{*}$ are compatible with the functors of tensor product and Hom of CDG-modules in the following sense. For any left CDG-modules $L$, $M$ and right CDG-module $N$ over $B$ there are natural isomorphisms

(25) $\phi_{!} N \otimes_{\phi_{!} B} \phi_{!} M \simeq \phi_{!}\left(N \otimes_{B} M\right)$ and $\operatorname{Hom}^{\phi_{!} B}\left(\phi_{!} L, \phi_{*} M\right) \simeq \phi_{*} \operatorname{Hom}^{B}(L, M)$.

For any left CDG-modules $L^{\prime}$ and $M^{\prime}$ over $\phi_{!} B$ there are natural isomorphisms

$$
\begin{aligned}
\phi^{*}\left(\phi_{!} N \otimes_{\phi_{!} B} M^{\prime}\right) & \simeq N \otimes_{B} \phi^{*} M^{\prime}, \\
\phi^{*} \operatorname{Hom}^{\phi_{!} B}\left(\phi_{!} L, M^{\prime}\right) & \simeq \operatorname{Hom}^{B}\left(L, \phi^{*} M^{\prime}\right), \\
\phi^{*} \operatorname{Hom}^{\phi_{!} B}\left(L^{\prime}, \phi_{*} M\right) & \simeq \operatorname{Hom}^{B}\left(\phi^{*} L^{\prime}, M\right) .
\end{aligned}
$$

It follows from the isomorphisms (26) that the functors $\phi_{\text {! }}$ preserve all the flatness and projectivity properties of CDG- and DG-modules previously considered in this paper, while the functors $\phi_{*}$ preserve the injectivity properties. Furthermore,

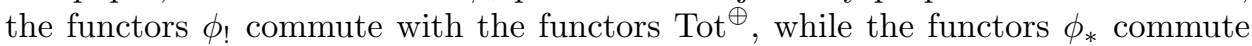
with the functors Tot ${ }^{\sqcap}$. Therefore, in view of the isomorphisms (25), for any $\Gamma$ graded DG-category $C$ and DG-modules $L, M$, and $N$ over it there are natural isomorphisms

$$
\begin{aligned}
& \operatorname{Tor}^{\phi_{!} C}\left(\phi_{!} N, \phi_{!} M\right) \simeq \phi_{!} \operatorname{Tor}^{C}(N, M) \text { and } \operatorname{Ext}_{\phi_{!} C}\left(\phi_{!} L, \phi_{*} M\right) \simeq \phi_{*} \operatorname{Ext}_{C}(L, M) \\
& \text { in } \mathrm{D}_{\Gamma^{\prime}}(k \text {-mod }) .
\end{aligned}
$$

Furthermore, the functor $\phi_{\text {! }}$ preserves tensor products of $k$-linear (C)DG-categories. Thus, assuming that the complexes of morphisms in the DG-category $C$ are h-flat complexes of $k$-modules, for any DG-module $M$ over $C \otimes_{k} C^{\text {op }}$ there are natural isomorphisms of the Hochschild (co)homology

$$
H H_{*}\left(\phi_{!} C, \phi_{!} M\right) \simeq \phi_{!} H H_{*}(C, M) \text { and } H H^{*}\left(\phi_{!} C, \phi_{*} M\right) \simeq \phi_{*} H H^{*}(C, M) .
$$

In particular, there is an isomorphism

$$
H H_{*}\left(\phi_{!} C\right) \simeq \phi_{!} H H_{*}(C)
$$

and a natural morphism

$$
H H^{*}\left(\phi_{!} C\right) \longrightarrow H H^{*}\left(\phi_{!} C, \phi_{*} C\right) \simeq \phi_{*} H H^{*}(C) .
$$

The latter morphism is an isomorphism when the kernel of the map $\phi: \Gamma \longrightarrow \Gamma^{\prime}$ is finite (so the functors $\phi_{!}$and $\phi_{*}$ are isomorphic).

The analogous results for (co)homology theories of the second kind hold under

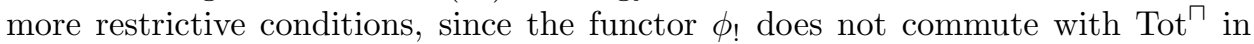
general nor does the functor $\phi_{*}$ commute with $\operatorname{Tot}^{\oplus}$. However, there are morphisms of functors $\phi_{!} \operatorname{Tot}^{\sqcap} \longrightarrow \operatorname{Tot}^{\sqcap} \phi_{!}$and $\operatorname{Tot}^{\oplus} \phi_{*} \longrightarrow \phi_{*} \operatorname{Tot}^{\oplus}$.

Hence for any $\Gamma$-graded CDG-category $B$ and CDG-modules $L, M$, and $N$ over it, there are natural morphisms

$$
\begin{aligned}
& \phi_{!} \operatorname{Tor}^{B}(N, M) \longrightarrow \operatorname{Tor}^{\phi_{!} B}\left(\phi_{!} N, \phi_{!} M\right), \\
& \operatorname{Ext}_{\phi ! B}\left(\phi_{!} L, \phi_{*} M\right) \longrightarrow \phi_{*} \operatorname{Ext}_{B}(L, M)
\end{aligned}
$$

in $\mathrm{D}_{\Gamma^{\prime}}(k$-mod). The morphisms (31)-(32) are always isomorphisms when the kernel of the map $\phi: \Gamma \longrightarrow \Gamma^{\prime}$ is finite. They are also isomorphisms when the derived functors in question can be computed using finite resolutions (cf. Section 3.3). So the morphism (31) is an isomorphism whenever one of the $\Gamma$-graded $B^{\#}$-modules $N^{\#}$ and $M^{\#}$ has finite flat dimension. The morphism (32) is an isomorphism 
whenever either the $\Gamma$-graded $B^{\#}$-module $L^{\#}$ has finite projective dimension or the $\Gamma$-graded $B^{\#}$-module has finite injective dimension.

Thus, assuming that the $\Gamma$-graded $k$-modules of morphisms in the category $B^{\#}$ are flat, for any CDG-module $M$ over $B \otimes_{k} B^{\text {op }}$ there are natural morphisms of Hochschild (co)homology

$$
\begin{gathered}
\phi_{!} H H_{*}^{I I}(B, M) \longrightarrow H H_{*}^{I I}\left(\phi_{!} B, \phi_{!} M\right) \\
H H^{I I, *}\left(\phi_{!} B, \phi_{*} M\right) \longrightarrow \phi_{*} H H^{I I, *}(B, M),
\end{gathered}
$$

which are always isomorphims when the kernel of the map $\phi: \Gamma \longrightarrow \Gamma^{\prime}$ is finite. The map (33) is an isomorphism whenever one of the $\Gamma$-graded $B^{\#} \otimes_{k} B^{\# \text { op }}$-modules $B^{\#}$ and $M^{\#}$ has finite flat dimension. The map (34) is an isomorphism whenever either the $\Gamma$-graded $B^{\#} \otimes_{k} B^{\# \text { op }}$-module $B^{\#}$ has finite projective dimension or the $\Gamma$-graded $B^{\#} \otimes_{k} B^{\# \text { op }}$-module $M^{\#}$ has finite injective dimension.

In particular, there is a natural map

$$
\phi_{!} H H_{*}^{I I}(B) \longrightarrow H H_{*}\left(\phi_{!} B\right),
$$

which is an isomorphism when either the kernel of the map $\phi: \Gamma \longrightarrow \Gamma^{\prime}$ is finite or the $\Gamma$-graded $B^{\#} \otimes_{k} B^{\# \text { op }}$-module $B^{\#}$ has finite flat dimension. There are also natural maps

$$
H H^{I I, *}\left(\phi_{!} B\right) \longrightarrow H H^{I I, *}\left(\phi_{!} B, \phi_{*} B\right) \longrightarrow \phi_{*} H H^{I I, *}(B),
$$

which are both isomorphisms when the kernel of the map $\phi: \Gamma \longrightarrow \Gamma^{\prime}$ is finite.

Given a $\Gamma^{\prime}$-graded $k$-linear CDG-category $B^{\prime}$, one can apply the functor $\phi^{*}$ to all of its precomplexes of morphisms, obtaining a $\Gamma$-graded $k$-linear CDG-category $\phi^{*} B^{\prime}$. To a (left or right) CDG-module $M^{\prime}$ over $B^{\prime}$ one can assign a CDGmodule $\phi^{*} M^{\prime}$ over $\phi^{*} B^{\prime}$. Assume that the map $\phi: \Gamma \longrightarrow \Gamma^{\prime}$ is surjective. Then the functors $\phi^{*}: Z^{0}\left(B^{\prime}-\bmod ^{\text {cdg }}\right) \longrightarrow Z^{0}\left(\phi^{*} B^{\prime}-\bmod ^{\text {cdg }}\right)$ and $Z^{0}\left(\bmod ^{\text {cdg }}-B^{\prime}\right) \longrightarrow$ $Z^{0}\left(\bmod ^{\mathrm{cdg}}-\phi^{*} B^{\prime}\right)$ are equivalences of abelian categories. For any left CDG-modules $L^{\prime}, M^{\prime}$ and right CDG-module $N^{\prime}$ over $B^{\prime}$ there are natural isomorphisms

$$
\begin{aligned}
\phi^{*} N^{\prime} \otimes_{\phi^{*} B^{\prime}} \phi^{*} M^{\prime} & \simeq \phi^{*}\left(N^{\prime} \otimes_{B^{\prime}} M^{\prime}\right), \\
\operatorname{Hom}^{\phi^{*} B^{\prime}}\left(\phi^{*} L^{\prime}, \phi^{*} M^{\prime}\right) & \simeq \phi^{*} \operatorname{Hom}^{B^{\prime}}\left(L^{\prime}, M^{\prime}\right) .
\end{aligned}
$$

Furthermore, the functor $\phi^{*}$ commutes with the functors $\operatorname{Tot}^{\oplus}$ and $\operatorname{Tot}^{\sqcap}$ when applied to polycomplexes with one grading by elements of the group $\Gamma^{\prime}$ and the remaining gradings by the integers.

Therefore, there are natural isomorphisms

$$
\begin{aligned}
\operatorname{Tor}^{\phi^{*} B^{\prime}, I I}\left(\phi^{*} N^{\prime}, \phi^{*} M^{\prime}\right) & \simeq \phi^{*} \operatorname{Tor}^{B^{\prime}, I I}\left(N^{\prime}, M^{\prime}\right), \\
\operatorname{Ext}_{\phi^{*} B^{\prime}}^{I I}\left(\phi^{*} N^{\prime}, \phi^{*} M^{\prime}\right) & \simeq \phi^{*} \operatorname{Ext}_{B^{\prime}}^{I I}\left(N^{\prime}, M^{\prime}\right)
\end{aligned}
$$

in $\mathrm{D}_{\Gamma}(k$-mod), and there are similar isomorphisms for the Tor and Ext of the first kind over a $k$-linear DG-category $C$.

There is a natural strict CDG-functor $\phi^{*} B^{\prime} \otimes_{k} \phi^{*} B^{\prime o p} \longrightarrow \phi^{*}\left(B^{\prime} \otimes_{k} B^{\prime o p}\right)$. So, assuming that the $\Gamma^{\prime}$-graded $k$-modules of morphisms in the category $B^{\prime \#}$ are flat, for any CDG-module $M^{\prime}$ over $B^{\prime} \otimes_{k} B^{\prime o p}$ there are natural maps

$$
\begin{gathered}
H H_{*}^{I I}\left(\phi^{*} B^{\prime}, \phi^{*} M^{\prime}\right) \longrightarrow \phi^{*} H H_{*}^{I I}\left(B^{\prime}, M^{\prime}\right), \\
\phi^{*} H H^{I I, *}\left(B^{\prime}, M^{\prime}\right) \longrightarrow H H^{I I, *}\left(\phi^{*} B^{\prime}, \phi^{*} M^{\prime}\right),
\end{gathered}
$$


and, in particular,

(42)

$$
H H_{*}^{I I}\left(\phi^{*} B^{\prime}\right) \longrightarrow \phi^{*} H H_{*}^{I I}\left(B^{\prime}\right) \quad \text { and } \quad \phi^{*} H H^{I I, *}\left(B^{\prime}\right) \longrightarrow H H^{I I, *}\left(\phi^{*} B^{\prime}\right) .
$$

One can see that the maps (40)- (42) are isomorphisms whenever the kernel $\Gamma^{\prime \prime}$ of the map $\phi: \Gamma \longrightarrow \Gamma^{\prime}$ is finite and its order $\left|\Gamma^{\prime \prime}\right|$ is invertible in $k$. Indeed, the CDG-category $\phi^{*} B^{\prime} \otimes_{k} \phi^{*} B^{\prime o p}$ is linear over the group ring $k\left[\Gamma^{\prime \prime}\right]$ of the abelian group $\Gamma^{\prime \prime}$, and the CDG-category $\phi^{*}\left(B^{\prime} \otimes_{K} B^{\prime o p}\right)$ is strictly equivalent (in fact, isomorphic) to $\left(\phi^{*} B^{\prime} \otimes_{k} \phi^{*} B^{\prime o p}\right) \otimes_{k\left[\Gamma^{\prime \prime}\right]} k$. The same assertions apply to the Hochschild (co)homology of the first kind of a $\Gamma^{\prime}$-graded DG-category $C$ whose complexes of morphisms are h-flat complexes of $k$-modules.

2.6. DG-category of CDG-modules. Let $B$ be a small $k$-linear CDG-category such that the $\Gamma$-graded $k$-modules $B^{\#}(X, Y)$ are flat for all objects $X, Y \in B$. Denote by $C=\bmod _{\mathrm{fgp}}^{\mathrm{cdg}}-B$ the DG-category of right CDG-modules over $B$, projective and finitely generated as $\Gamma$-graded $B^{\#}$-modules, and by $D=\bmod _{\mathrm{fgp}}^{\mathrm{qdg}}-B$ the CDGcategory of right QDG-modules over $B$ satisfying the same condition. The results below also apply to finitely generated free modules in place of finitely generated projective ones.

There are strict $k$-linear CDG-functors $R: B \longrightarrow D$ and $I: C \longrightarrow D$, and, moreover, these CDG-functors are pseudo-equivalences of CDG-categories (see Section [1.5). Strictly speaking, the categories $C$ and $D$ as we have defined them are only essentially small rather than small, i.e., they are strictly equivalent to small CDG-categories. So from now on we will tacitly assume that $C$ and $D$ have been replaced with their small full subcategories containing at least one object in every isomorphism class and such that the functors $R$ and $I$ are still defined.

The pseudo-equivalences $R$ and $I$ induce equivalences between the DG-categories of (left or right) CDG-modules over the CDG-categories $B, C$, and $D$. Let $N$ be a right CDG-module and $L, M$ be left CDG-modules over $B$. Denote by $N_{C}, N_{D}$, $L_{C}, L_{D}$, etc. the corresponding CDG-modules over $C$ and $D$ (which are defined uniquely up to a unique isomorphism). By the results of Section 2.2 (see (7)-(8)), the CDG-functors $R$ and $I$ induce isomorphisms

$\operatorname{Tor}^{B, I I}(N, M) \longrightarrow \operatorname{Tor}^{D, I I}\left(N_{D}, M_{D}\right)$ and $\operatorname{Tor}^{C, I I}\left(N_{C}, M_{C}\right) \longrightarrow \operatorname{Tor}^{D, I I}\left(N_{D}, M_{D}\right)$,

$$
\operatorname{Ext}_{D}^{I I}\left(L_{D}, M_{D}\right) \longrightarrow \operatorname{Ext}_{B}^{I I}(L, M) \text { and } \operatorname{Ext}_{D}^{I I}\left(L_{D}, M_{D}\right) \longrightarrow \operatorname{Ext}_{C}^{I I}\left(L_{C}, M_{C}\right) .
$$

There are also the induced pseudo-equivalences $R \otimes R^{\mathrm{op}}: B \otimes_{k} B^{\mathrm{op}} \longrightarrow D \otimes_{k}$ $D^{\mathrm{op}}$ and $I \otimes I^{\mathrm{op}}: C \otimes_{k} C^{\mathrm{op}} \longrightarrow D \otimes_{k} D^{\mathrm{op}}$. These pseudo-equivalences induce equivalences between the DG-categories of CDG-modules over the CDG-categories $B \otimes_{k} B^{\mathrm{op}}, C \otimes_{k} C^{\mathrm{op}}$, and $D \otimes_{k} D^{\mathrm{op}}$. In particular, the CDG-module $B$ over $B \otimes_{k} B^{\mathrm{op}}$ corresponds to the CDG-module $C$ over $C \otimes_{k} C^{\text {op }}$ and to the CDG-module $D$ over $D \otimes_{k} D^{\text {op }}$ under these equivalences of DG-categories. Indeed, the closed morphisms $B \longrightarrow R^{*} D$ and $C \longrightarrow I^{*} D$ of CDG-modules over $B \otimes_{k} B^{\text {op }}$ and $C \otimes_{k} C^{\text {op }}$ induced by the functors $R$ and $I$ are isomorphisms, since the functors $R^{\#}$ and $I^{\#}$ are fully faithful.

Let $M$ be a CDG-module over $B \otimes_{k} B^{\text {op }}$. Denote by $M_{C}$ and $M_{D}$ the corresponding CDG-modules over $C \otimes_{k} C^{\text {op }}$ and $D \otimes_{k} D^{\text {op }}$. Then the CDG-functors 
$R \otimes R^{\mathrm{op}}$ and $I \otimes I^{\mathrm{op}}$ induce isomorphisms (see (16), (19))

$$
\begin{array}{rll}
H H_{*}^{I I}(B, M) \longrightarrow H H_{*}^{I I}\left(D, M_{D}\right) & \text { and } & H H_{*}^{I I}\left(C, M_{C}\right) \longrightarrow H H_{*}^{I I}\left(D, M_{D}\right) ; \\
H H^{I I, *}\left(D, M_{D}\right) \longrightarrow H H^{I I, *}(B, M) & \text { and } & H H^{I I, *}\left(D, M_{D}\right) \longrightarrow H H^{I I, *}\left(C, M_{C}\right) .
\end{array}
$$

In particular, we obtain natural isomorphisms

$$
H H_{*}^{I I}(B) \simeq H H_{*}^{I I}(C) \text { and } H H^{I I, *}(B) \simeq H H^{I I, *}(C) .
$$

This is a generalization of [18, Theorem 2.14].

When the ring $k$ has finite weak homological dimension, any $\Gamma$-graded complex of flat $k$-modules is flat. So if the $\Gamma$-graded $k$-modules of morphisms in the category $B^{\#}$, and hence also in the category $C^{\#}$, are flat, then the complexes of morphisms in the DG-category $C$ are h-flat. Thus, both the Hochschild (co)homologies of the first and the second kinds are defined for the DG-category $C$, and therefore the natural maps between the Hochschild (co)homologies of the first and second kinds of the DG-category $C$ with coefficients in any DG-module over $C \otimes_{k} C^{\text {op }}$ are defined.

\section{Derived CATEgories OF THE SECOND KIND}

In this section we interpret, under certain homological dimension assumptions, the Ext and Tor of the second kind over a CDG-category in terms of the derived categories of the second kind of CDG-modules over it. This allows us to obtain sufficient conditions for an isomorphism of the Hochschild (co)homology of the first and second kinds for a DG-category, and in particular, for the DG-category $C$ of CDG-modules over a CDG-category $B$, projective and finitely generated as $\Gamma$-graded $B^{\#}$-modules.

3.1. Conventional derived category. Given a DG-category $D$, the additive category $H^{0}(D)$ has a natural triangulated category structure provided that a zero object and all shift and cones exist in $D$. In particular, for any small DG-category $C$ the categories $H^{0}\left(C-\bmod ^{\mathrm{dg}}\right)$ and $H^{0}\left(\bmod ^{\mathrm{dg}}-C\right)$ are triangulated. These are called the homotopy categories of (left and right) DG-modules over $C$.

A (left or right) DG-module $M$ over $C$ is said to be acyclic if the complexes $M(X)$ are acyclic for all objects $X \in C$, i.e., $H(M)=0$. Acyclic DG-modules form thick subcategories, closed under both infinite directs sums and infinite products, in the homotopy categories of DG-modules. The quotient categories by these thick subcategories are called the (conventional) derived categories (of the first kind) of DG-modules over $C$ and denoted by $\mathrm{D}\left(C\right.$-mod $\left.{ }^{\mathrm{dg}}\right)$ and $\mathrm{D}\left(\bmod ^{\mathrm{dg}}-C\right)$.

The full subcategory of h-projective DG-modules

$$
H^{0}\left(C-\bmod ^{\mathrm{dg}}\right)_{\mathrm{prj}} \subset H^{0}\left(C-\bmod ^{\mathrm{dg}}\right)
$$

is a triangulated subcategory whose functor to $\mathrm{D}\left(C-\bmod ^{\mathrm{dg}}\right)$ is an equivalence of categories [6], and the same applies to the full subcategory of h-injective DG-modules $H^{0}\left(C \text {-mod }{ }^{\mathrm{dg}}\right)_{\mathrm{inj}} \subset H^{0}\left(C\right.$-mod $\left.{ }^{\mathrm{dg}}\right)$. To prove these results, one notices first of all that any projective object in the exact category $Z^{0}\left(C\right.$-mod $\left.{ }^{\mathrm{dg}}\right)$ is an h-projective DG-module, and similarly for injectives (see Section 2.1 for the discussion of this exact category and its projective/injective objects). Let $P_{\bullet}$ be a left projective resolution of a DG-module $M$ in $Z^{0}\left(C\right.$-mod). Then the total DG-module of $P_{\bullet}$, constructed by taking infinite direct sums along the diagonals, is an h-projective 
DG-module quasi-isomorphic to $M$. Similarly, if $J^{\bullet}$ is a right injective resolution of a DG-module $M$ in $Z^{0}\left(C\right.$-mod $\left.{ }^{\mathrm{dg}}\right)$, then the total DG-module of $J^{\bullet}$, constructed by taking infinite products along the diagonals, is an h-injective DG-module quasiisomorphic to $M$ [15, Section 1].

Furthermore, the full subcategory of h-flat DG-modules

$$
H^{0}\left(C-\bmod ^{\mathrm{dg}}\right)_{\mathrm{fl}} \subset H^{0}\left(C-\bmod ^{\mathrm{dg}}\right)
$$

is a triangulated subcategory whose quotient category by its intersection with thick subcategory of acyclic DG-modules is equivalent to $\mathrm{D}\left(C\right.$-mod $\left.{ }^{\mathrm{dg}}\right)$. This follows from the above result for h-projective DG-modules and the fact that any h-flat DG-module is h-projective. The same applies to the full subcategory of h-flat right DG-modules $H^{0}\left(\bmod ^{\mathrm{dg}}-C\right)_{\mathrm{fl}} \subset H^{0}\left(\bmod ^{\mathrm{dg}}-C\right)$.

Let $k$ be a commutative ring and $C$ be a small $k$-linear DG-category. Restricting the triangulated functor of two arguments (see (2) )

$$
\operatorname{Hom}^{C}: H^{0}\left(C-\bmod ^{\mathrm{dg}}\right)^{\text {op }} \times H^{0}\left(C-\bmod ^{\mathrm{dg}}\right) \longrightarrow \mathrm{D}(k \text {-mod })
$$

to the full subcategory of h-projective DG-modules in the first argument, one obtains a functor that factors through the derived category in the second argument, providing the derived functor

$$
\operatorname{Ext}_{C}: \mathrm{D}\left(C-\bmod ^{\mathrm{dg}}\right)^{\mathrm{op}} \times \mathrm{D}\left(C-\bmod ^{\mathrm{dg}}\right) \longrightarrow \mathrm{D}(k-\bmod ) .
$$

Alternatively, restricting the functor $\operatorname{Hom}^{C}$ to the full subcategory of h-injective DG-modules in the second argument, one obtains a functor that factors through the derived category in the first argument, leading to the same derived functor $\operatorname{Ext}_{C}$. The composition of this derived functor with the localization functor $Z^{0}\left(C-\bmod ^{\mathrm{dg}}\right) \longrightarrow \mathrm{D}\left(C-\bmod ^{\mathrm{dg}}\right)$ is isomorphic to the derived functor $\operatorname{Ext}_{C}$ constructed in Section 2.1. For any left DG-modules $L$ and $M$ over $C$ there is a natural isomorphism

$$
H^{*} \operatorname{Ext}_{C}(L, M) \simeq \operatorname{Hom}_{\mathrm{D}\left(C-\bmod ^{\mathrm{dg}}\right)}(L, M[*]) .
$$

Analogously, restricting the triangulated functor of two arguments (see (11))

$$
\otimes_{C}: H^{0}\left(\bmod ^{\mathrm{dg}}-C\right) \times H^{0}\left(C-\bmod ^{\mathrm{dg}}\right) \longrightarrow \mathrm{D}(k-\bmod )
$$

to the full subcategory of h-flat DG-modules in the first argument, one obtains a functor that factors through the Cartesian product of the derived categories, providing the derived functor

$$
\text { Tor }^{C}: \mathrm{D}\left(\bmod ^{\mathrm{dg}}-C\right) \times \mathrm{D}\left(C-\bmod ^{\mathrm{dg}}\right) \longrightarrow \mathrm{D}(k-\bmod ) .
$$

The same derived functor can be obtained by restricting the functor $\otimes_{C}$ to the full subcategory of h-flat DG-modules in the second argument. Up to composing with the localization functors $Z^{0}\left(\bmod ^{\mathrm{dg}}-C\right) \longrightarrow \mathrm{D}\left(\bmod ^{\mathrm{dg}}-C\right)$ and $Z^{0}\left(C-\bmod ^{\mathrm{dg}}\right) \longrightarrow$ $\mathrm{D}\left(C-\bmod ^{\mathrm{dg}}\right)$, this is the same derived functor Tor ${ }^{C}$ that was constructed in Section 2.1

3.2. Derived categories of the second kind. Let $B$ be a small CDG-category. As in Section [3.1, the homotopy categories of $C D G$-modules $H^{0}\left(B\right.$-mod $\left.{ }^{\mathrm{cdg}}\right)$ and $H^{0}\left(\bmod ^{\mathrm{cdg}}-B\right)$ over $B$ are naturally triangulated. Given a short exact sequence $0 \longrightarrow K^{\prime} \longrightarrow K \longrightarrow K^{\prime \prime} \longrightarrow 0$ in the abelian category $Z^{0}\left(B-\bmod ^{\text {cdg }}\right)$, one can consider it as a finite complex of closed morphisms in the DG-category $B$-mod ${ }^{\text {cdg }}$ and take the corresponding total object in $B-\bmod ^{\text {cdg }}$ [15, Section 1.2]. 
A left CDG-module over $B$ is called absolutely acyclic if it belongs to the minimal thick subcategory of $H^{0}\left(B\right.$-mod $\left.{ }^{\text {cdg }}\right)$ containing the total CDG-modules of exact triples of CDG-modules. The quotient category of $H^{0}\left(B\right.$-mod $\left.{ }^{\text {cdg }}\right)$ by the thick subcategory of absolutely acyclic CDG-modules is called the absolute derived category of left CDG-modules over $B$ and is denoted by $\mathrm{D}^{\text {abs }}\left(B\right.$-mod $\left.{ }^{\text {cdg }}\right)$ [15, Section 3.3].

A left CDG-module over $B$ is called coacyclic if it belongs to the minimal triangulated subcategory of $H^{0}\left(B\right.$-mod $\left.{ }^{\text {cdg }}\right)$ containing the total CDG-modules of exact triples of CDG-modules and closed under infinite direct sums. The quotient category of $H^{0}\left(B\right.$-mod $\left.{ }^{\text {cdg }}\right)$ by the thick subcategory of coacyclic CDG-modules is called the coderived category of left CDG-modules over $B$ and is denoted by $\mathrm{D}^{\mathrm{co}}\left(B-\bmod ^{\mathrm{cdg}}\right)$.

The definition of a contraacyclic CDG-module is dual to the previous one. A left CDG-module over $B$ is called contraacyclic if it belongs to the minimal triangulated subcategory of $H^{0}\left(B\right.$-mod $\left.{ }^{\text {cdg }}\right)$ containing the total CDG-modules of exact triples of CDG-modules and closed under infinite products. The quotient category of $H^{0}\left(B-\bmod ^{\text {cdg }}\right)$ by the thick subcategory of contraacyclic CDG-modules is called the contraderived category of left CDG-modules over $B$ and denoted by $\mathrm{D}^{\mathrm{ctr}}\left(B\right.$-mod $\left.{ }^{\mathrm{cdg}}\right)$.

Coacyclic, contraacyclic, and absolutely acyclic right CDG-modules are defined in an analogous way. The corresponding exotic derived (quotient) categories are denoted by $\mathrm{D}^{\mathrm{co}}\left(\bmod ^{\mathrm{cdg}}-B\right), \mathrm{D}^{\mathrm{ctr}}\left(\bmod ^{\mathrm{cdg}}-B\right)$, and $\mathrm{D}^{\mathrm{abs}}\left(\bmod ^{\mathrm{cdg}}-B\right)$.

We will use the similar notation $\mathrm{D}^{\mathrm{co}}\left(C\right.$-mod $\left.{ }^{\mathrm{dg}}\right), \mathrm{D}^{\mathrm{ctr}}\left(C\right.$-mod $\left.{ }^{\mathrm{dg}}\right)$, etc., in the particular case of the coderived, contraderived, and absolutely derived categories of DG-modules over a small DG-category $C$. Notice that any coacyclic or contraacyclic DG-module is acyclic. The converse is not true [15, Examples 3.3].

Furthermore, given an exact subcategory in the abelian category of $\Gamma$-graded

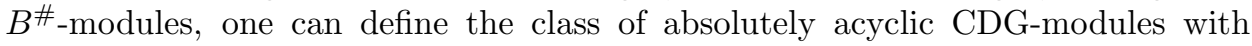
respect to this exact subcategory (or the DG-category of CDG-modules whose underlying $\Gamma$-graded modules belong to this exact subcategory). For this purpose, one considers exact triples of CDG-modules whose underlying $\Gamma$-graded modules belong to the exact subcategory, takes their total CDG-modules, and uses them to generate a thick subcategory of the homotopy category of all CDG-modules whose underlying $\Gamma$-graded modules belong to the exact subcategory. When the exact subcategory is closed under infinite direct sums (resp., infinite products), the class of coacyclic (resp., contraacyclic) CDG-modules with respect to this exact subcategory is defined. Taking the quotient category, one obtains the coderived, contraderived, or absolute derived category of CDG-modules with the given restriction on the underlying $\Gamma$-graded modules.

We will be particularly interested in the coderived and absolute derived categories of CDG-modules over $B$ whose underlying $\Gamma$-graded $B^{\#}$-modules are flat or have finite flat dimension (see Section 2.5 for the terminology). Denote the DG-categories of right CDG-modules over $B$ with such restrictions on the underlying $\Gamma$-graded modules by $\bmod _{\mathrm{fl}}^{\mathrm{cdg}}-B$ and $\bmod _{\mathrm{ffd}}^{\mathrm{cdg}}-B$, and their absolute derived categories by $\mathrm{D}^{\text {abs }}\left(\bmod _{\mathrm{fl}}^{\mathrm{cdg}}-B\right)$ and $\mathrm{D}^{\text {abs }}\left(\bmod _{\mathrm{ffd}}^{\mathrm{cdg}}-B\right)$. The coderived category of $\bmod _{\mathrm{fl}}^{\mathrm{cdg}}-B$, defined as explained above, is denoted by $\mathrm{D}^{\mathrm{co}}\left(\bmod _{\mathrm{fl}}^{\mathrm{cdg}}-B\right)$.

The definition of the coderived category $\mathrm{D}^{\mathrm{co}}\left(\bmod _{\mathrm{ffd}}^{\mathrm{cdg}}-B\right)$ requires a little more care because the class of modules of finite flat dimension is not closed under infinite direct sums; only the classes of modules of flat dimension not exceeding a fixed 
number $d$ are. Let us call a CDG-module $N$ over $B d$-flat if its underlying $\Gamma$ graded $B^{\#}$-module $M^{\#}$ has flat dimension not greater than $d$. Define an object $N \in H^{0}\left(\bmod _{\mathrm{ffd}}^{\mathrm{cdg}}-B\right)$ to be coacyclic with respect to $\bmod _{\mathrm{ffd}}^{\mathrm{cdg}}-B$ if there exists an integer $d \geqslant 0$ such that the CDG-module $N$ is coacyclic with respect to the DGcategory of $d$-flat CDG-modules over $B$. The coderived category $\mathrm{D}^{\mathrm{co}}\left(\bmod _{\mathrm{ffd}}^{\mathrm{cdg}}-B\right)$ is the quotient category of the homotopy category $H^{0}\left(\bmod _{\mathrm{ffd}}^{\mathrm{cdg}}-B\right)$ by the thick subcategory of CDG-modules coacyclic with respect to $\bmod _{\mathrm{ffd}}^{\mathrm{cdg}}-B$.

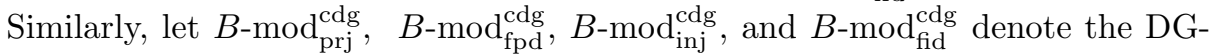
categories of left CDG-modules over $B$ whose underlying $\Gamma$-graded $B^{\#}$-modules are projective, of finite projective dimension, injective, and of finite injective dimension, respectively. The notation for the homotopy categories and exotic derived categories of these DG-categories is similar to the above. The definition of the coderived category $\mathrm{D}^{\mathrm{co}}\left(B\right.$-mod $\left.\mathrm{f}_{\mathrm{fpd}}^{\mathrm{cdg}}\right)$ and the contraderived category $\mathrm{D}^{\mathrm{ctr}}\left(B-\bmod _{\mathrm{fid}}^{\mathrm{cdg}}\right)$ involves the same subtle point as discussed above. It is dealt with in the same way, i.e., the class of CDG-modules coacyclic with respect to $B$ - $\bmod _{\mathrm{fpd}}^{\mathrm{cdg}}$ or contraacyclic with respect to $B$ - $\bmod _{\text {fid }}^{\text {cdg }}$ is defined as the union of the classes of CDG-modules coacyclic or contraacyclic with respect to the category of modules of the projective or injective dimension bounded by a fixed integer.

\section{Theorem.}

(a) The functors $\mathrm{D}^{\mathrm{co}}\left(\bmod _{\mathrm{fl}}^{\mathrm{cdg}}-B\right) \longrightarrow \mathrm{D}^{\mathrm{co}}\left(\bmod _{\mathrm{ffd}}^{\mathrm{cdg}}-B\right)$ and $\mathrm{D}^{\mathrm{abs}}\left(\bmod _{\mathrm{fl}}^{\mathrm{cdg}}-B\right) \longrightarrow$ $\mathrm{D}^{\mathrm{abs}}\left(\bmod _{\mathrm{ffd}}^{\mathrm{cdg}}-B\right)$ induced by the embedding $\bmod _{\mathrm{fl}}^{\mathrm{cdg}}-B \longrightarrow \bmod _{\mathrm{fdd}}^{\mathrm{cdg}}-B$ are equivalences of triangulated categories.

(b) The functors $H^{0}\left(B-\bmod _{\mathrm{prj}}^{\mathrm{cdg}}\right) \longrightarrow \mathrm{D}^{\mathrm{abs}}\left(B-\bmod _{\mathrm{fpd}}^{\mathrm{cdg}}\right) \longrightarrow \mathrm{D}^{\mathrm{co}}\left(B-\bmod _{\mathrm{fpd}}^{\mathrm{cdg}}\right)$, the first of which is induced by the embedding $B-\bmod _{\mathrm{prj}}^{\mathrm{cdg}} \longrightarrow B$-mod $\operatorname{dpd}_{\mathrm{fpd}}^{\mathrm{cdg}}$ and the second is the localization functor, are equivalences of triangulated categories.

(c) The functors $H^{0}\left(B\right.$ - $\left.\bmod _{\mathrm{inj}}^{\mathrm{cdg}}\right) \longrightarrow \mathrm{D}^{\mathrm{abs}}\left(B-\bmod _{\mathrm{fid}}^{\mathrm{cdg}}\right) \longrightarrow \mathrm{D}^{\mathrm{ctr}}\left(B-\bmod _{\mathrm{fid}}^{\mathrm{cdg}}\right)$, the first of which is induced by the embedding $B-\bmod _{\mathrm{inj}}^{\mathrm{cdg}} \longrightarrow B-\bmod _{\mathrm{fid}}^{\mathrm{cdg}}$ and the second is the localization functor, are equivalences of triangulated categories.

Proof. The first equivalence in part (b) is easy to prove. By [15, Theorem 3.5(b)], CDG-modules that are projective as $\Gamma$-graded modules are semiorthogonal to any contraacyclic CDG-modules in $H^{0}\left(B\right.$-mod $\left.{ }^{\mathrm{cdg}}\right)$. The construction of [15, proof of Theorem 3.6] shows that any object of $H^{0}\left(B-\bmod _{\mathrm{fpd}}^{\text {cdg }}\right)$ is a cone of a morphism from a CDG-module that is absolutely acyclic with respect to $B-\bmod _{\mathrm{fpd}}^{\text {cdg }}$ to an object of $H^{0}\left(B-\bmod _{\mathrm{prj}}^{\mathrm{cdg}}\right)$. It follows that the functor $H^{0}\left(B-\bmod _{\mathrm{prj}}^{\mathrm{cdg}}\right) \longrightarrow \mathrm{D}^{\text {abs }}\left(B-\bmod _{\mathrm{fpd}}^{\mathrm{cdg}}\right)$ is an equivalence of triangulated categories. Moreover, any object of $H^{0}\left(B\right.$-mod fpd $\left._{\text {cdg }}^{\text {de }}\right)$ that is contraacyclic with respect to $B$-mod ${ }^{\text {cdg }}$ is absolutely acyclic with respect to $B-\bmod _{\mathrm{fpd}}^{\mathrm{cdg}}$.

To prove the second equivalence in part (b), it suffices to show that any object of $H^{0}\left(B-\bmod _{\mathrm{prj}}^{\mathrm{cdg}}\right)$ that is coacyclic with respect to $B$ - $\bmod _{\mathrm{fpd}}^{\mathrm{cdg}}$ is coacyclic with respect to $B-\bmod _{\mathrm{prj}}^{\mathrm{cdg}}$ (as any object of the latter kind is clearly contractible). The proof of this is analogous to the proof of part (a) below. It follows that any CDG-module coacyclic with respect to $B$ - $\bmod _{\mathrm{fpd}}^{\text {cdg }}$ is absolutely acyclic with respect to $B$ - $\bmod _{\mathrm{fpd}}^{\text {cdg }}$. The proof of part (c) is analogous to the proof of part (b) up to the duality. 
To prove part (a), notice that the same construction from [15, proof of Theorem 3.6] allows us to present any object of $H^{0}\left(\bmod _{\mathrm{ffd}}^{\mathrm{cdg}}-B\right)$ as a cone of a morphism from a CDG-module that is absolutely acyclic with respect to $\bmod _{\mathrm{ffd}}^{\mathrm{cdg}}-B$ to an object of $H^{0}\left(\bmod _{\mathrm{fl}}^{\mathrm{cdg}}-B\right)$. By [15, Lemma 1.6], it remains to show that any object of $H^{0}\left(\bmod _{\mathrm{fl}}^{\mathrm{cdg}}-B\right)$ that is coacyclic (absolutely acyclic) with respect to $\bmod _{\mathrm{ffd}}^{\mathrm{cdg}}-B$ is coacyclic (absolutely acyclic) with respect to $\bmod _{\mathrm{fl}}^{\mathrm{cdg}}-B$.

We follow the idea of the proof of [14, Theorem 7.2.2]. Given an integer $d \geqslant 0$, let us call a $d$-flat right CDG-module $N$ over $B d$-coacyclic if it is coacyclic with respect to the exact category of $d$-flat CDG-modules over $B$. We will show that for any $d$-coacyclic CDG-module $N$ there exists a $(d-1)$-coacyclic CDG-module $L$ together with a surjective closed morphism of CDG-modules $L \longrightarrow N$ whose kernel $K$ is also $(d-1)$-coacyclic. It will follow that any $(d-1)$-flat $d$-coacyclic CDG-module $N$ is $(d-1)$-coacyclic, since the total CDG-module of the exact triple $K \longrightarrow L \longrightarrow M$ is $(d-1)$-coacyclic, as is the cone of the morphism $K \longrightarrow L$. By induction we will conclude that any 0 -flat $d$-coacyclic CDG-module is 0-coacyclic. The argument for absolutely acyclic CDG-modules will be similar.

To prove that a $d$-coacyclic CDG-module can be presented as a quotient of a $(d-1)$-coacyclic CDG-module by a $(d-1)$-coacyclic CDG-submodule, we will first construct such a presentation for totalizations of exact triples of $d$-flat CDGmodules, and then check that the class of $d$-flat CDG-modules presentable in this form is stable under taking cones and homotopy equivalences.

Lemma A. Let $N$ be the total $C D G$-module of an exact triple of d-flat CDGmodules $N^{\prime} \longrightarrow N^{\prime \prime} \longrightarrow N^{\prime \prime \prime}$. Then there exists a surjective closed morphism onto $N$ from a 0-coacyclic CDG-module $P$ with a $(d-1)$-coacyclic kernel $K$.

Proof. Choose projective objects $P^{\prime}$ and $P^{\prime \prime \prime}$ in the abelian category of CDGmodules $Z^{0}\left(\bmod ^{\mathrm{cdg}}-B\right)$ (see Section 2.2) such that there are surjective morphisms $P^{\prime} \longrightarrow N^{\prime}$ and $P^{\prime \prime \prime} \longrightarrow N^{\prime \prime \prime}$. Then there exists a surjective morphism from the exact triple CDG-modules $P^{\prime} \longrightarrow P^{\prime \prime}=P^{\prime} \oplus P^{\prime \prime \prime} \longrightarrow P^{\prime \prime \prime}$ onto the exact triple $N^{\prime} \longrightarrow N^{\prime \prime} \longrightarrow N^{\prime \prime \prime}$. Let $K^{\prime} \longrightarrow K^{\prime \prime} \longrightarrow K^{\prime \prime \prime}$ be the kernel of this morphism of exact triples. Then the CDG-modules $P^{(i)}$ are 0 -flat, while the CDG-modules $K^{(i)}$ are $(d-1)$-flat. Therefore, the total CDG-module $P$ of the exact triple $P^{\prime} \longrightarrow P^{\prime \prime} \longrightarrow P^{\prime \prime \prime}$ is 0-coacyclic (in fact, 0-flat and contractible), while the total CDG-module $K$ of the exact triple $K^{\prime} \longrightarrow K^{\prime \prime} \longrightarrow K^{\prime \prime \prime}$ is $(d-1)$-coacyclic.

Lemma B. (a) Let $K^{\prime} \longrightarrow L^{\prime} \longrightarrow N^{\prime}$ and $K^{\prime \prime} \longrightarrow L^{\prime \prime} \longrightarrow N^{\prime \prime}$ be exact triples of $C D G$-modules such that the $C D G$-modules $K^{\prime}, L^{\prime}, K^{\prime \prime}, L^{\prime \prime}$ are $(d-1)$-coacyclic, and let $N^{\prime} \longrightarrow N^{\prime \prime}$ be a closed morphism of CDG-modules. Then there exists an exact triple of $C D G$-modules $K \longrightarrow L \longrightarrow N$ with $N=\operatorname{cone}\left(N^{\prime} \rightarrow N^{\prime \prime}\right)$ and $(d-1)$-coacylic $C D G$-modules $K$ and $L$.

(b) In the situation of (a), assume that the morphism $N^{\prime} \longrightarrow N^{\prime \prime}$ is injective with a d-flat cokernel $N_{0}$. Then there exists an exact triple of $C D G$-modules $K_{0} \longrightarrow$ $L_{0} \longrightarrow N_{0}$ with $(d-1)$-coacyclic $C D G$-modules $K_{0}$ and $L_{0}$.

Proof. Denote by $L^{\prime \prime \prime}$ the CDG-module $L^{\prime} \oplus L^{\prime \prime}$. Then there is the embedding of a direct summand $L^{\prime} \longrightarrow L^{\prime \prime \prime}$ and the surjective closed morphism of CDG-modules $L^{\prime \prime \prime} \longrightarrow N^{\prime \prime}$ whose components are the composition $L^{\prime} \longrightarrow N^{\prime} \longrightarrow N^{\prime \prime}$ and the surjective morphism $L^{\prime \prime} \longrightarrow N^{\prime \prime}$. These two morphisms form a commutative square with the morphisms $L^{\prime} \longrightarrow N^{\prime}$ and $N^{\prime} \longrightarrow N^{\prime \prime}$. The kernel $K^{\prime \prime \prime}$ of the morphism 
$L^{\prime \prime \prime} \longrightarrow N^{\prime \prime}$ is the middle term of an exact triple of CDG-modules $K^{\prime \prime} \longrightarrow K^{\prime \prime \prime} \longrightarrow$ $L^{\prime}$. Since the CDG-modules $K^{\prime \prime}$ and $L^{\prime}$ are $(d-1)$-coacyclic, the CDG-module $K^{\prime \prime \prime}$ is $(d-1)$-coacyclic, too. Set $L=\operatorname{cone}\left(L^{\prime} \rightarrow L^{\prime \prime \prime}\right)$ and $K=\operatorname{cone}\left(K^{\prime} \rightarrow K^{\prime \prime \prime}\right)$.

To prove part (b), notice that the above morphisms of CDG-modules $L^{\prime} \longrightarrow L^{\prime \prime \prime}$ and $K^{\prime} \longrightarrow K^{\prime \prime \prime}$ are injective; denote their cokernels by $L_{0}$ and $K_{0}$. Then the CDG-module $L_{0} \simeq L^{\prime \prime}$ is $(d-1)$-coacyclic. In the assumptions of part (b), the CDG-module $K_{0}$ is the kernel of the surjective morphism $L_{0} \longrightarrow N_{0}$, so it is $(d-1)$-flat. Hence it follows from the exact triple $K^{\prime} \longrightarrow K^{\prime \prime \prime} \longrightarrow K_{0}$ that $K_{0}$ is $(d-1)$-coacyclic.

Lemma C. For any contractible d-flat $C D G$-module $N$ there exists an exact triple $K \longrightarrow P \longrightarrow N$ with with a 0 -flat contractible $C D G$-module $P$ and $a(d-1)$-flat contractible $C D G$-module $K$.

Proof. It is easy to see using the explicit description of projective objects in $Z^{0}\left(\bmod ^{\mathrm{cdg}}-B\right)$ given in Section 2.2 that any projective CDG-module is contractible. Let $p: P \longrightarrow N$ be a surjective morphism onto $N$ from a projective CDG-module $P$. Let $t: N \longrightarrow N$ be a contracting homotopy for $N$ and $\theta: P \longrightarrow P$ be a contracting homotopy for $P$. Then $p \theta-t p: P \longrightarrow N$ is a closed morphism of CDG-modules of degree -1 . Since $P$ is projective and $p$ is surjective, there exists a closed morphism $b: P \longrightarrow P$ of degree -1 such that $p \theta-t p=p b$. Hence $\theta-b$ is another contracting homotopy for $P$ making a commutative square with the contracting homotopy $t$ and the morphism $p$. It follows that the restriction of $\theta-b$ on the kernel $K$ of the morphism $p$ is a contracting homotopy for the CDG-module $K$.

Lemma D. Let $N \longrightarrow N^{\prime}$ be a homotopy equivalence of d-flat CDG-modules, and suppose that there is an exact triple of $C D G$-modules $K^{\prime} \longrightarrow L^{\prime} \longrightarrow N^{\prime}$ with $(d-1)$-coacyclic $C D G$-modules $K^{\prime}$ and $L^{\prime}$. Then there exists an exact triple of $C D G$-modules $K \longrightarrow L \longrightarrow N$ with $(d-1)$-coacyclic $C D G$-modules $K$ and $L$.

Proof. The cone of the morphism $N \longrightarrow N^{\prime}$, being a contractible $d$-flat CDGmodule, is the cokernel of an injective morphism of $(d-1)$-coacyclic CDG-modules by Lemma C. By Lemma B(a), the cocone $N^{\prime \prime}$ of the morphism $N^{\prime} \longrightarrow \operatorname{cone}(N \rightarrow$ $N^{\prime}$ ) can also be presented in such form. The CDG-module $N^{\prime \prime}$ is isomorphic to the direct sum of the CDG-module $N$ and the cocone $N^{\prime \prime \prime}$ of the identity endomorphism of the CDG-module $N^{\prime}$. The CDG-module $N^{\prime \prime \prime}$ can also be presented in the desired form. Hence, by Lemma B(b), so can the cokernel $N$ of the injective morphism $N^{\prime \prime \prime} \longrightarrow N^{\prime \prime}$.

It is clear that the property of a CDG-module to be presentable as the quotient of a $(d-1)$-coacyclic CDG-module by a $(d-1)$-coacyclic CDG-submodule is stable under infinite direct sums. The assertion that all $d$-coacyclic CDG-modules can be presented in such form now follows from Lemmas A, B(a), and D.

In particular, it follows from part (b) of the Theorem that there is a natural fully faithful functor $\mathrm{D}^{\mathrm{co}}\left(B-\bmod _{\mathrm{fpd}}^{\mathrm{cdg}}\right) \longrightarrow \mathrm{D}^{\mathrm{ctr}}\left(B\right.$-mod $\left.{ }^{\mathrm{cdg}}\right)$. Indeed, the functor $H^{0}\left(B-\bmod _{\mathrm{prj}}^{\mathrm{cdg}}\right) \simeq \mathrm{D}^{\mathrm{abs}}\left(B-\bmod _{\mathrm{fpd}}^{\mathrm{cdg}}\right) \longrightarrow \mathrm{D}^{\mathrm{ctr}}\left(B-\bmod ^{\mathrm{cdg}}\right)$ is fully faithful by 15 , Theorem 3.5(b) and Lemma 1.3]. Similarly, the functor $H^{0}\left(B-\bmod _{\mathrm{inj}}^{\mathrm{cdg}}\right) \simeq \mathrm{D}^{\mathrm{abs}}$ $\left(B-\bmod _{\mathrm{fid}}^{\mathrm{cdg}}\right) \longrightarrow \mathrm{D}^{\mathrm{co}}\left(B-\bmod ^{\mathrm{cdg}}\right)$ is fully faithful by [15, Theorem 3.5(a)], so there is a natural fully faithful functor $\mathrm{D}^{\mathrm{ctr}}\left(B-\bmod _{\mathrm{fid}}^{\mathrm{cdg}}\right) \longrightarrow \mathrm{D}^{\mathrm{co}}\left(B-\bmod ^{\mathrm{cdg}}\right)$. 
3.3. Derived functors of the second kind. Let $B$ be a small $k$-linear CDG-category and $L$ be a left CDG-module over $B$ such that the $\Gamma$-graded left $B^{\#}$-module $L^{\#}$ has finite projective dimension. Then the CDG-module $L$ admits a finite left resolution $P_{\bullet}$ in the abelian category $Z^{0}\left(B-\bmod ^{\text {cdg }}\right)$ such that the $\Gamma$-graded $B^{\#}{ }_{-}$ modules $P_{i}^{\#}$ are projective. This resolution can be used to compute the functor $\operatorname{Ext}_{B}^{I I}(L,-)$ as defined in Section 2.2.

On the other hand, let $P$ denote the total CDG-module of the finite complex of CDG-modules $P_{\bullet}$. Then for any left CDG-module $M$ over $B$ the $k$-module of morphisms from $L$ into $M$ in $\mathrm{D}^{\text {abs }}\left(B\right.$ - $\left.\bmod ^{\text {cdg }}\right)$ or $\mathrm{D}^{\text {ctr }}\left(B-\bmod ^{\mathrm{cdg}}\right)$ is isomorphic to the $k$-module of morphisms from $P$ into $M$ in the homotopy category $H^{0}\left(B\right.$-mod $\left.{ }^{\text {cdg }}\right)$ [15, Theorem 3.5(b) and Lemma 1.3]. Thus,

$$
H^{*} \operatorname{Ext}_{B}^{I I}(L, M) \simeq \operatorname{Hom}_{D^{\text {abs }}\left(B-\bmod ^{\text {cdg }}\right)}(L, M[*]) \simeq \operatorname{Hom}_{D^{\text {ctr }}\left(B-\bmod ^{\text {cdg }}\right)}(L, M[*]) .
$$

Similar isomorphisms

$$
H^{*} \operatorname{Ext}_{B}^{I I}(L, M) \simeq \operatorname{Hom}_{\mathrm{D}^{\mathrm{abs}}\left(B-\bmod ^{\mathrm{cdg}}\right)}(L, M[*]) \simeq \operatorname{Hom}_{\mathrm{D}^{\mathrm{co}}\left(B-\bmod ^{\mathrm{cdg}}\right)}(L, M[*])
$$

hold if one assumes, instead of the condition on $L^{\#}$, that the $\Gamma$-graded $B^{\#}$-module $M^{\#}$ has finite injective dimension.

One can lift these comparison results from the level of cohomology modules to the level of the derived category $\mathrm{D}(k$-mod) in the following way. Consider the functor (see (60) )

$$
\operatorname{Hom}^{B}: H^{0}\left(B-\bmod ^{\text {cdg }}\right)^{\text {op }} \times H^{0}\left(B-\bmod ^{\text {cdg }}\right) \longrightarrow \mathrm{D}(k \text {-mod })
$$

and restrict it to the subcategory $H^{0}\left(B-\bmod _{\mathrm{prj}}^{\mathrm{cdg}}\right)^{\text {op }}$ in the first argument. This restriction factors through the contraderived category $\mathrm{D}^{\text {ctr }}\left(B\right.$-mod $\left.{ }^{\text {cdg }}\right)$ in the second argument. Taking into account Theorem 3.2 (b), we obtain a right derived functor

$$
\mathrm{D}^{\mathrm{co}}\left(B-\bmod _{\mathrm{fpd}}^{\mathrm{cdg}}\right)^{\mathrm{op}} \times \mathrm{D}^{\mathrm{ctr}}\left(B-\bmod ^{\mathrm{cdg}}\right) \longrightarrow \mathrm{D}(k-\bmod ) .
$$

The composition of this derived functor with the localization functors $Z^{0}\left(B\right.$-mod $\left.\operatorname{dpd}_{\mathrm{fpd}}^{\text {cdg }}\right)$

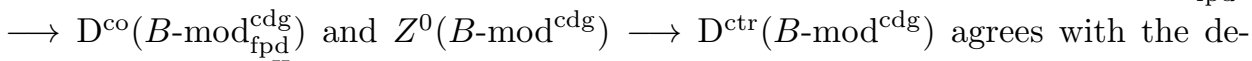
rived functor $\operatorname{Ext}_{B}^{I I}$ where the former is defined.

In the same way one can use Theorem 3.2 (c) to construct a right derived functor

$$
\mathrm{D}^{\mathrm{co}}\left(B-\bmod ^{\mathrm{cdg}}\right)^{\mathrm{op}} \times \mathrm{D}^{\mathrm{ctr}}\left(B-\bmod _{\text {fid }}^{\mathrm{cdg}}\right) \longrightarrow \mathrm{D}(k-\bmod ),
$$

which agrees with the functor $\operatorname{Ext}_{B}^{I I}$ where the former is defined, up to composing with the localization functors.

Analogously, consider the functor (see (5) )

$$
\otimes_{B}: H^{0}\left(\bmod ^{\mathrm{cdg}}-B\right) \times H^{0}\left(B-\bmod ^{\mathrm{cdg}}\right) \longrightarrow \mathrm{D}(k-\bmod )
$$

and restrict it to the subcategory $H^{0}\left(\bmod _{\mathrm{fl}}^{\mathrm{cdg}}-B\right)$ in the first argument. This restriction factors through the Cartesian product $\mathrm{D}^{\mathrm{co}}\left(\bmod _{\mathrm{fl}}^{\mathrm{cdg}}-B\right) \times \mathrm{D}^{\mathrm{co}}\left(B-\bmod ^{\mathrm{cdg}}\right)$. Indeed, the tensor product of a CDG-module that is flat as a $\Gamma$-graded module with a coacyclic CDG-module is clearly acyclic, as is the tensor product of a CDGmodule coacyclic with respect to $\bmod _{\mathrm{fl}}^{\mathrm{cdg}}-B$ with any CDG-module over $B$. Taking into account Theorem $\underline{3.2}$ (a), we obtain a left derived functor

$$
\mathrm{D}^{\mathrm{co}}\left(\bmod _{\mathrm{ffd}}^{\mathrm{cdg}}-B\right) \times \mathrm{D}^{\mathrm{co}}\left(B-\bmod ^{\mathrm{cdg}}\right) \longrightarrow \mathrm{D}(k-\bmod ) .
$$


Up to composing with the localization functors $Z^{0}\left(\bmod _{\mathrm{fdd}}^{\mathrm{cdg}}-B\right) \longrightarrow \mathrm{D}^{\mathrm{co}}\left(\bmod _{\mathrm{ffd}}^{\mathrm{cdg}}-B\right)$ and $Z^{0}\left(B-\bmod ^{\text {cdg }}\right) \longrightarrow \mathrm{D}^{\mathrm{co}}\left(B-\bmod ^{\text {cdg }}\right)$, this derived functor agrees with the derived functor $\operatorname{Tor}^{B, I I}$, where the former is defined. To see this, it suffices, as above, to choose for a CDG-module $N \in \bmod _{\mathrm{ffd}}^{\mathrm{cdg}}-B$ a finite left resolution $Q \bullet$ in the abelian category $Z^{0}\left(\bmod ^{\mathrm{cdg}}-B\right)$ such that the $\Gamma$-graded $B^{\#}$-modules $Q_{i}^{\#}$ are flat.

Remark. The functor Tor ${ }^{B, I I}$ factors through the Cartesian product of the absolute derived categories, defining a triangulated functor of two arguments

$$
\mathrm{D}^{\mathrm{abs}}\left(\bmod ^{\mathrm{cdg}}-B\right) \times \mathrm{D}^{\mathrm{abs}}\left(B-\bmod ^{\mathrm{cdg}}\right) \longrightarrow \mathrm{D}(k-\bmod )
$$

15. Section 3.12]. This functor agrees with the functor (48) in the sense that the composition of the former with the functor $\mathrm{D}^{\mathrm{abs}}\left(\bmod _{\mathrm{fdd}}^{\mathrm{cdg}}-B\right) \longrightarrow \mathrm{D}^{\mathrm{abs}}\left(\bmod ^{\mathrm{cdg}}-B\right)$ in the first argument is isomorphic to the composition of the latter with the functors $\mathrm{D}^{\mathrm{abs}}\left(\bmod _{\mathrm{ffd}}^{\mathrm{cdg}}-B\right) \longrightarrow \mathrm{D}^{\mathrm{co}}\left(\bmod _{\mathrm{ffd}}^{\mathrm{cdg}}-B\right)$ and $\mathrm{D}^{\mathrm{abs}}\left(B-\bmod ^{\mathrm{cdg}}\right) \longrightarrow \mathrm{D}^{\mathrm{co}}\left(B\right.$-mod $\left.{ }^{\mathrm{cdg}}\right)$ in the first and second arguments, respectively. Analogously, the functor $\mathrm{Ext}_{B}^{I I}$ descends to a triangulated functor of two arguments

$$
\mathrm{D}^{\mathrm{abs}}\left(B-\bmod ^{\mathrm{cdg}}\right)^{\mathrm{op}} \times \mathrm{D}^{\mathrm{abs}}\left(B-\bmod ^{\mathrm{cdg}}\right) \longrightarrow \mathrm{D}(k \text {-mod }),
$$

which agrees with the functors (46) and (47) in a similar sense.

3.4. Comparison of the two theories. Let $C$ be a small $k$-linear DG-category. Recall (see Section 3.1) the notation $H^{0}\left(C-\bmod ^{\mathrm{dg}}\right)_{\mathrm{prj}}$ for the homotopy category of h-projective left DG-modules over $C$. As in Section 3.2, let $C$-mod prj and $H^{0}\left(C\right.$-mod prj $\left.^{\mathrm{dg}}\right)$ denote the DG-category of left DG-modules over $C$ whose underlying $\Gamma$-graded $C^{\#}$-modules are projective, and its homotopy category. Finally, denote by $H^{0}\left(C-\bmod _{\text {prj }}^{\mathrm{dg}}\right)_{\text {prj }}$ the full triangulated subcategory in $H^{0}\left(C-\bmod _{\text {prj }}^{\mathrm{dg}}\right)$ formed by the h-projective left DG-modules over $C$ whose underlying $\Gamma$-graded $C^{\# \text {-modules }}$ are projective. The functors

$$
H^{0}\left(C-\bmod _{\text {prj }}^{\mathrm{dg}}\right)_{\mathrm{prj}} \longrightarrow H^{0}\left(C-\bmod ^{\mathrm{dg}}\right)_{\mathrm{prj}} \longrightarrow \mathrm{D}\left(C-\bmod ^{\mathrm{dg}}\right)
$$

are equivalences of triangulated categories. Moreover, for any left DG-module $L$ over $C$ there exists a DG-module $P \in H^{0}\left(C \text {-mod } \operatorname{mprj}_{\mathrm{prj}}^{\mathrm{dg}}\right)_{\mathrm{pr}}$ together with a quasiisomorphism $P \longrightarrow L$ of DG-modules over $C$ (see [6] or [15, Section 1]).

The equivalence of categories $H^{0}\left(C-\bmod _{\mathrm{prj}}^{\mathrm{dg}}\right)_{\mathrm{prj}} \longrightarrow \mathrm{D}\left(C-\bmod ^{\mathrm{dg}}\right)$ factors as the following composition:

$$
H^{0}\left(C-\bmod _{\mathrm{prj}}^{\mathrm{dg}}\right)_{\mathrm{prj}} \longrightarrow H^{0}\left(C-\bmod _{\mathrm{prj}}^{\mathrm{dg}}\right) \longrightarrow \mathrm{D}^{\mathrm{co}}\left(C-\bmod _{\mathrm{fpd}}^{\mathrm{dg}}\right) \longrightarrow \mathrm{D}\left(C-\bmod ^{\mathrm{dg}}\right),
$$

where the middle arrow is also an equivalence of categories (by Theorem $3.2(\mathrm{~b})$ ). Besides, there is the localization functor $\mathrm{D}^{\mathrm{ctr}}\left(C-\bmod ^{\mathrm{dg}}\right) \longrightarrow \mathrm{D}\left(C-\bmod ^{\mathrm{dg}}\right)$. This allows us to construct a natural morphism

$$
\operatorname{Ext}_{C}^{I I}(L, M) \longrightarrow \operatorname{Ext}_{C}(L, M)
$$

in $\mathrm{D}(k$-mod $)$ for any objects $L \in \mathrm{D}^{\mathrm{co}}\left(C\right.$-mod $\left.\bmod _{\mathrm{fpd}}^{\mathrm{dg}}\right)$ and $M \in \mathrm{D}^{\mathrm{ctr}}\left(C\right.$-mod $\left.{ }^{\mathrm{dg}}\right)$.

Specifically, for a given DG-module $L$ choose a DG-module $F \in H^{0}\left(C\right.$-mod $\left.\operatorname{prj}^{\mathrm{dg}}\right)$ and a closed morphism $F \longrightarrow L$ with a cone coacyclic with respect to $C-\bmod _{\mathrm{fpd}}^{\mathrm{dg}}$. Next, for the DG-module $F$ choose a DG-module $P \in H^{0}\left(C \text {-mod } \operatorname{prj}_{\mathrm{pr}}^{\mathrm{dg}}\right)_{\mathrm{prj}}$ together with a quasi-isomorphism $P \longrightarrow F$. Then the complex $\operatorname{Hom}^{C}(F, M)$ represents the object $\operatorname{Ext}_{C}^{I I}(L, M)$, the complex $\operatorname{Hom}^{C}(P, M)$ represents the object $\operatorname{Ext}_{C}(L, M)$, 
and the morphism $\operatorname{Hom}^{C}(F, M) \longrightarrow \operatorname{Hom}^{C}(P, M)$, induced by the morphism $P \longrightarrow$ $F$, represents the desired morphism (49). This morphism does not depend on the choices of the objects $F$ and $P$.

To see that the comparison morphism (49) coincides with the morphism (10) constructed in Section 2.2 choose a projective resolution $P_{\bullet}$ of the object $L$ in the exact category $Z^{0}\left(C\right.$-mod $\left.{ }^{\mathrm{dg}}\right)$. Then both the resolution $P_{\bullet}$ and its finite canonical truncation $\tau_{\geqslant-d} P$. for $d$ large enough are resolutions of $L$ that can be used to compute $\operatorname{Ext}_{C}^{I I}(L, M)$ by the procedure of Section 2.2 , while the whole resolution $P_{\bullet}$ can also be used to compute $\operatorname{Ext}_{C}(L, M)$ by the procedure of Section 2.1 Set $F$ to be the total DG-module of the finite complex of DG-modules $\tau_{\geqslant-d} P$. and $P$ the total DG-module of the complex of DG-modules $P_{\bullet}$, constructed by taking infinite direct sums along the diagonals. Then the morphism of complexes $\operatorname{Hom}^{C}(F, M) \longrightarrow \operatorname{Hom}^{C}(P, M)$ represents both the morphisms (10) and (49) in $\mathrm{D}(k$-mod).

Analogously, denote by $H^{0}\left(C-\bmod _{\mathrm{inj}}^{\mathrm{dg}}\right)_{\text {inj }}$ the full triangulated subcategory in $H^{0}\left(C\right.$-mod inj $\left.^{\mathrm{dg}}\right)$ formed by the h-injective left DG-modules over $C$ whose underlying $\Gamma$-graded $C^{\#}$-modules are injective. Here, as above, the notation $H^{0}\left(C \text {-mod }{ }^{\mathrm{dg}}\right)_{\mathrm{inj}}$ for the category of h-injective DG-modules comes from Section 3.1, while the notation $C$-mod dinj and $H^{0}\left(C-\bmod _{\text {inj }}^{\mathrm{dg}}\right)$ for the categories of DG-modules whose underlying $\Gamma$-graded modules are injective is similar to that in Section 3.2 , The functors

$$
H^{0}\left(C-\bmod _{\mathrm{inj}}^{\mathrm{dg}}\right)_{\mathrm{inj}} \longrightarrow H^{0}\left(C-\bmod ^{\mathrm{dg}}\right)_{\mathrm{inj}} \longrightarrow \mathrm{D}\left(C \text {-mod }{ }^{\mathrm{dg}}\right)
$$

are equivalences of triangulated categories. Moreover, for any left DG-module $M$

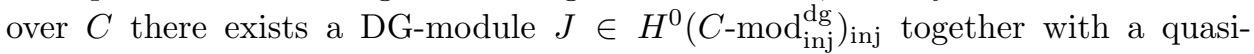
isomorphism $M \longrightarrow J$ of CDG-modules over $C$.

The equivalence of categories $H^{0}\left(C-\bmod _{\mathrm{inj}}^{\mathrm{dg}}\right)_{\mathrm{inj}} \longrightarrow \mathrm{D}\left(C\right.$-mod $\left.{ }^{\mathrm{dg}}\right)$ factors as the following composition:

$$
H^{0}\left(C-\bmod _{\mathrm{inj}}^{\mathrm{dg}}\right)_{\mathrm{inj}} \longrightarrow H^{0}\left(C-\bmod _{\mathrm{inj}}^{\mathrm{dg}}\right) \longrightarrow \mathrm{D}^{\mathrm{ctr}}\left(C-\bmod _{\mathrm{fid}}^{\mathrm{dg}}\right) \longrightarrow \mathrm{D}\left(C-\bmod ^{\mathrm{dg}}\right),
$$

where the middle arrow is also an equivalence of categories (by Theorem $3.2(\mathrm{c})$ ). Besides, there is the localization functor $\mathrm{D}^{\mathrm{co}}\left(C-\bmod ^{\mathrm{dg}}\right) \longrightarrow \mathrm{D}\left(C-\bmod ^{\mathrm{dg}}\right)$. This allows us to construct a natural morphism

$$
\operatorname{Ext}_{C}^{I I}(L, M) \longrightarrow \operatorname{Ext}_{C}(L, M)
$$

in $\mathrm{D}(k$-mod $)$ for any objects $L \in \mathrm{D}^{\mathrm{co}}\left(C\right.$-mod $\left.{ }^{\mathrm{dg}}\right)$ and $M \in \mathrm{D}^{\mathrm{ctr}}\left(C\right.$-mod $\left.\operatorname{mid}_{\mathrm{fg}}^{\mathrm{dg}}\right)$.

Specifically, for a given DG-module $M$ choose a DG-module $I \in H^{0}\left(C\right.$ - $\left.\bmod _{\text {inj }}^{\mathrm{dg}}\right)$ and a closed morphism $M \longrightarrow I$ with a cone contraacyclic with respect to $C$ - $\bmod _{\text {fid }}^{\text {dg }}$. Next, for the DG-module $I$ choose a DG-module $J \in H^{0}\left(C \text {-mod }{ }_{\text {inj }}^{\mathrm{dg}}\right)_{\text {inj }}$ together with a quasi-isomorphism $I \longrightarrow J$. Then the complex $\operatorname{Hom}^{C}(L, I)$ represents the object $\operatorname{Ext}_{C}^{I I}(L, M)$, the complex $\operatorname{Hom}_{C}(L, J)$ represents the object $\operatorname{Ext}_{C}(L, M)$, and the morphism $\operatorname{Hom}_{C}(L, I) \longrightarrow \operatorname{Hom}_{C}(L, J)$ represents the desired morphism (50). This comparison morphism agrees with the comparison morphism (10) from Section 2.2. where the former is defined.

Finally, denote by $H^{0}\left(\bmod _{\mathrm{fl}}^{\mathrm{dg}}-C\right)_{\mathrm{fl}}$ the full triangulated subcategory in $H^{0}\left(\bmod _{\mathrm{fl}}^{\mathrm{dg}}-C\right)$ formed by h-flat right DG-modules over $C$ whose underlying $\Gamma$ graded $C^{\#}$-modules are flat. As above, $H^{0}\left(\bmod ^{\mathrm{dg}}-C\right)_{\mathrm{f}}$ is the homotopy category of h-flat right DG-modules over $C$, while $\bmod _{\mathrm{fl}}^{\mathrm{dg}}-C$ and $H^{0}\left(\bmod _{\mathrm{fl}}^{\mathrm{dg}}-C\right)$ denote the 
DG-category of right DG-modules whose underlying $\Gamma$-graded $C^{\#}$-modules are flat, and its homotopy category.

The functors between the quotient categories of $H^{0}\left(\bmod _{\mathrm{fl}}^{\mathrm{dg}}-C\right)_{\mathrm{fl}}$ and $H^{0}\left(\bmod ^{\mathrm{dg}}-C\right)_{\mathrm{fl}}$ by their intersections with the thick subcategory of acyclic DGmodules and the derived category $\mathrm{D}\left(\bmod ^{\mathrm{dg}}-C\right)$ are equivalences of triangulated categories. Moreover, for any right DG-module $N$ over $C$ there exists a DG-module $Q \in H^{0}\left(\bmod _{\mathrm{fl}}^{\mathrm{dg}}-C\right)_{\mathrm{fl}}$ together with a quasi-isomorphism of DG-modules $Q \longrightarrow N$ [15, Section 1.6].

The localization functor $H^{0}\left(\bmod _{\mathrm{fl}}^{\mathrm{dg}}-C\right)_{\mathrm{fl}} \longrightarrow \mathrm{D}\left(\bmod ^{\mathrm{dg}}-C\right)$ factors into the composition

$$
H^{0}\left(\bmod _{\mathrm{fl}}^{\mathrm{dg}}-C\right)_{\mathrm{fl}} \longrightarrow H^{0}\left(\bmod _{\mathrm{fl}}^{\mathrm{dg}}-C\right) \longrightarrow \mathrm{D}^{\mathrm{co}}\left(\bmod _{\mathrm{ffd}}^{\mathrm{dg}}-C\right) \longrightarrow \mathrm{D}\left(\bmod ^{\mathrm{dg}}-C\right)
$$

(the middle arrow being described by Theorem 3.2(a)). There is also the localization functor $\mathrm{D}^{\mathrm{co}}\left(C-\bmod ^{\mathrm{dg}}\right) \longrightarrow \mathrm{D}\left(C-\bmod ^{\mathrm{dg}}\right)$. This allows us to construct a natural morphism

$$
\operatorname{Tor}^{C}(N, M) \longrightarrow \operatorname{Tor}^{C, I I}(N, M)
$$

in $\mathrm{D}(k$-mod $)$ for any objects $N \in \mathrm{D}^{\mathrm{co}}\left(\bmod _{\mathrm{ffd}^{-}}^{\mathrm{dg}} C\right)$ and $M \in \mathrm{D}^{\mathrm{co}}\left(C-\bmod ^{\mathrm{dg}}\right)$ in the same way as above.

Specifically, for a given DG-module $N$ choose a DG-module $F \in H^{0}\left(\bmod _{\mathrm{fl}}^{\mathrm{dg}}-C\right)$ and a closed morphism $F \longrightarrow N$ with a cone coacyclic with respect to $\bmod _{\mathrm{ffd}}^{\mathrm{dg}}-C$. Next, for the DG-module $F$ choose a DG-module $Q \in H^{0}\left(\bmod _{\mathrm{ff}}^{\mathrm{dg}}-C\right)_{\mathrm{fl}}$ together with a quasi-isomorphism $Q \longrightarrow F$. Then the complex $F \otimes_{C} M$ represents the object $\operatorname{Tor}^{C, I I}(N, M)$, the complex $Q \otimes_{C} M$ represents the object $\operatorname{Tor}^{C}(N, M)$, and the morphism $Q \otimes_{C} M \longrightarrow F \otimes_{C} M$ represents the desired morphism (51). This comparison morphism agrees with morphism (9) from Section 2.2, where the former is defined.

Proposition. (a) The natural morphism $\operatorname{Tor}^{C}(N, M) \longrightarrow \operatorname{Tor}^{C, I I}(N, M)$ is an isomorphism whenever the $\Gamma$-graded $C^{\#}$-module $N^{\#}$ has finite flat dimension and there exists a closed morphism $Q \longrightarrow N$ into $N$ from a $D G$-module $Q \in H^{0}\left(\bmod _{\mathrm{fl}}^{\mathrm{dg}}-C\right)_{\mathrm{fl}}$ with a cone that is coacyclic with respect to $\bmod _{\mathrm{ffd}}^{\mathrm{dg}}-C$.

(b) The natural morphism $\operatorname{Ext}_{C}^{I I}(L, M) \longrightarrow \operatorname{Ext}_{C}(L, M)$ is an isomorphism whenever the $\Gamma$-graded $C^{\#}$-module $L^{\#}$ has finite projective dimension and the object $L \in H^{0}\left(C-\bmod _{\mathrm{fpd}}^{\mathrm{dg}}\right)$ belongs to the triangulated subcategory generated by $H^{0}\left(C-\bmod _{\mathrm{prj}}^{\mathrm{dg}}\right)_{\mathrm{prj}}$ and the subcategory of objects coacyclic with respect to $C$ - $\bmod _{\mathrm{fpd}}^{\mathrm{dg}}$. Equivalently, the latter conclusion holds whenever the $\Gamma$-graded $C^{\#}$-module $L^{\#}$ has finite projective dimension and the object $L \in \mathrm{D}^{\mathrm{ctr}}\left(C\right.$-mod $\left.{ }^{\mathrm{dg}}\right)$ belongs to the image of the functor $H^{0}\left(C \text {-mod }{ }^{\mathrm{dg}}\right)_{\mathrm{prj}} \longrightarrow \mathrm{D}^{\mathrm{ctr}}\left(C-\bmod ^{\mathrm{dg}}\right)$.

(c) The natural morphism $\operatorname{Ext}_{C}^{I I}(L, M) \longrightarrow \operatorname{Ext}_{C}(L, M)$ is also an isomorphism if the $\Gamma$-graded $C^{\#}$-module $M^{\#}$ has finite injective dimension and the object $M \in$ $H^{0}\left(C-\bmod _{\text {fid }}^{\mathrm{dg}}\right)$ belongs to the triangulated subcategory generated by $H^{0}\left(C-\bmod _{\mathrm{inj}}^{\mathrm{dg}}\right)_{\mathrm{inj}}$ and the subcategory of objects contraacyclic with respect to $C$ - $\bmod _{\mathrm{fid}}^{\mathrm{dg}}$.

Equivalently, the latter conclusion holds whenever the $\Gamma$-graded $C^{\#}$-module $M^{\#}$ has finite injective dimension and the object $M \in \mathrm{D}^{\mathrm{co}}\left(C\right.$-mod $\left.{ }^{\mathrm{dg}}\right)$ belongs to the image of the functor $H^{0}\left(C-\bmod ^{\mathrm{dg}}\right)_{\mathrm{inj}} \longrightarrow \mathrm{D}^{\mathrm{co}}\left(C-\bmod ^{\mathrm{dg}}\right)$. 
Notice that the equivalence of categories $H^{0}\left(C-\bmod ^{\mathrm{dg}}\right)_{\mathrm{prj}} \simeq \mathrm{D}\left(C\right.$-mod $\left.{ }^{\mathrm{dg}}\right)$ identifies the functor $H^{0}\left(C \text {-mod }{ }^{\mathrm{dg}}\right)_{\mathrm{prj}} \longrightarrow \mathrm{D}^{\mathrm{ctr}}\left(C\right.$-mod $\left.{ }^{\mathrm{dg}}\right)$ with the functor left adjoint to the localization functor $\mathrm{D}^{\mathrm{ctr}}\left(C\right.$-mod $\left.{ }^{\mathrm{dg}}\right) \longrightarrow \mathrm{D}\left(C\right.$-mod $\left.{ }^{\mathrm{dg}}\right)$. Analogously, the equivalence of categories $H^{0}\left(C-\bmod ^{\mathrm{dg}}\right)_{\mathrm{inj}} \simeq \mathrm{D}\left(C-\bmod ^{\mathrm{dg}}\right)$ identifies the functor $H^{0}\left(C-\bmod ^{\mathrm{dg}}\right)_{\mathrm{inj}} \longrightarrow \mathrm{D}^{\mathrm{co}}\left(C-\bmod ^{\mathrm{dg}}\right)$ with the functor right adjoint to the localization functor $\mathrm{D}^{\mathrm{co}}\left(C-\bmod ^{\mathrm{dg}}\right) \longrightarrow \mathrm{D}\left(C-\bmod ^{\mathrm{dg}}\right)$.

Before we prove the proposition, let us introduce some more notation. The triangulated category of (C)DG-modules coacyclic (resp., contraacyclic) with respect to a given DG-category of (C)DG-modules $D$ will be denoted by $\mathrm{Ac}^{\mathrm{co}}(D)$ (resp., $\left.\operatorname{Ac}^{\text {ctr }}(D)\right)$. So $\operatorname{Ac}^{\mathrm{co}}(D)$ and $\operatorname{Ac}^{\mathrm{ctr}}(D)$ are triangulated subcategories of $H^{0}(D)$. Similarly, $\operatorname{Ac}^{\text {abs }}(D)$ denotes the triangulated subcategory of absolutely acyclic (C)DGmodules. Finally, given a DG-category $C$, we denote by $\operatorname{Ac}\left(C\right.$-mod $\left.{ }^{\mathrm{dg}}\right)$ and $\mathrm{Ac}\left(\bmod ^{\mathrm{dg}}-C\right)$ the full subcategories of acyclic DG-modules in the homotopy categories $H^{0}\left(C-\bmod ^{\mathrm{dg}}\right)$ and $H^{0}\left(\bmod ^{\mathrm{dg}}-C\right)$.

Proof. Part (a) follows immediately from the above construction of the morphism (51). To prove the first assertion of part (b), notice that any morphism from an object of $H^{0}\left(C-\bmod _{\mathrm{prj}}^{\mathrm{dg}}\right)_{\mathrm{prj}}$ to an object of $\mathrm{Ac}^{\mathrm{co}}\left(C-\bmod _{\mathrm{fpd}}^{\mathrm{dg}}\right)$ vanishes in $H^{0}\left(C-\bmod ^{\mathrm{dg}}\right)$. In fact, any morphism from an object of $H^{0}\left(C-\bmod _{\mathrm{prj}}^{\mathrm{dg}}\right)$ to an object of $\operatorname{Ac}^{\mathrm{co}}\left(C\right.$ - $\left.\bmod _{\mathrm{fpd}}^{\mathrm{dg}}\right)$ vanishes, and any morphism from an object of $H^{0}\left(C \text {-mod }{ }^{\mathrm{dg}}\right)_{\mathrm{prj}}$ to an object of $\mathrm{Ac}\left(C\right.$-mod $\left.{ }^{\mathrm{dg}}\right)$ vanishes in the homotopy category. By the standard properties of semiorthogonal decompositions (see, e.g., [15, Lemma 1.3]), it follows that any object $L$ in the triangulated subcategory generated by $H^{0}\left(C \text {-mod } \mathrm{prj}_{\mathrm{prj}}^{\mathrm{dg}}\right)_{\mathrm{prj}}$ and $\mathrm{Ac}^{\mathrm{co}}\left(C-\bmod _{\mathrm{fpd}}^{\mathrm{dg}}\right)$ in $H^{0}(C$-mod $\mathrm{fpd})$ admits a closed morphism $P \longrightarrow L$ from an object $P \in H^{0}\left(C \text {-mod } \text { prj }_{\text {prj }}^{\mathrm{dg}}\right)_{\mathrm{pr}}$ with a cone in $\mathrm{Ac}^{\mathrm{co}}\left(C\right.$ - $\left.\bmod _{\mathrm{fpd}}^{\mathrm{dg}}\right)$.

To prove the equivalence of the two conditions in part (b), notice that, by the same semiorthogonality lemma, a DG-module $L \in C$ - $\bmod _{\mathrm{fpd}}^{\mathrm{dg}}$ belongs to the triangulated subcategory generated by $H^{0}\left(C-\bmod _{\mathrm{prj}}^{\mathrm{dg}}\right)_{\mathrm{prj}}$ and $\mathrm{Ac}^{\mathrm{co}}\left(C-\bmod _{\mathrm{fpd}}^{\mathrm{dg}}\right)$ in $H^{0}\left(C-\bmod _{\mathrm{fpd}}^{\mathrm{dg}}\right)$ if and only if, as an object of $\mathrm{D}^{\mathrm{co}}\left(C-\bmod _{\mathrm{fpd}}^{\mathrm{dg}}\right)$, it belongs to the image of $H^{0}\left(C-\bmod _{\text {prj }}^{\mathrm{dg}}\right)_{\text {prj }}$ in $\mathrm{D}^{\mathrm{co}}\left(C-\bmod _{\mathrm{fpd}}^{\mathrm{dg}}\right)$. Then use the concluding remarks in Section 3.2 Part (c) is similar to part (b) up to the duality.

In particular, if the left homological dimension of the $\Gamma$-graded category $C^{\#}$ is finite (see Section 2.5 for the terminology), then the classes of coacyclic, contraacyclic, and absolutely acyclic left DG-modules over $C$ coincide [15, Theorem 3.6(a)]. In this case, for any left DG-modules $L$ and $M$ over $C$ the morphism of $\Gamma$-graded $k$-modules $H^{*} \operatorname{Ext}_{C}^{I I}(L, M) \longrightarrow H^{*} \operatorname{Ext}_{C}(L, M)$ is naturally identified with the morphism

$$
\operatorname{Hom}_{\mathrm{D}^{\mathrm{abs}}\left(C-\bmod ^{\mathrm{dg}}\right)}(L, M) \longrightarrow \operatorname{Hom}_{\mathrm{D}\left(C-\bmod ^{\mathrm{dg}}\right)}(L, M) .
$$

So if the class of absolutely acyclic left DG-modules also coincides with the class of acyclic DG-modules, then the natural morphism $\operatorname{Ext}_{C}^{I I}(L, M) \longrightarrow \operatorname{Ext}_{C}(L, M)$ is an isomorphism for any DG-modules $L$ and $M$ over $C$.

Analogously, if the weak homological dimension of the $\Gamma$-graded category $C^{\#}$ is finite and the category $H^{0}\left(\bmod _{\mathrm{fl}}^{\mathrm{dg}}-C\right)$ coincides with its full subcategory 
$H^{0}\left(\bmod _{\mathrm{fl}}^{\mathrm{dg}}-C\right)_{\mathrm{fl}}$, then the natural morphism $\operatorname{Tor}^{C}(N, M) \longrightarrow \operatorname{Tor}^{C, I I}(N, M)$ is an isomorphism for any DG-modules $N$ and $M$ over $C$. This follows from part (a) of the Proposition.

3.5. Comparison for the DG-category of CDG-modules. Let $B$ be a small $k$-linear CDG-category and $C=\bmod _{\mathrm{fgp}}^{\mathrm{cdg}}-B$ the DG-category of right CDG-modules over $B$, projective and finitely generated as $\Gamma$-graded $B^{\#}$-modules. The results below also apply, mutatis mutandis, to finitely generated free modules in place of finitely generated projective ones.

The DG-categories of (left or right) CDG-modules over $B$ and DG-modules over $C$ are naturally equivalent. Let $M_{C}$ denote the DG-module over $C$ corresponding to a CDG-module $M$ over $B$ (see Sections 1.5] and 2.6). Denote by $B$ - $\bmod _{\mathrm{fgp}}^{\text {cdg }}$ the DG-category of left CDG-modules over $B$, finitely generated and projective as $\Gamma$ graded $B^{\#}$-modules. Let $k^{\vee}$ be an injective cogenerator of the abelian category of $k$ modules. For example, one can take $k^{\vee}=k$ when $k$ is a field, or $k^{\vee}=\operatorname{Hom}_{\mathbb{Z}}(k, \mathbb{Q} / \mathbb{Z})$ for any ring $k$.

Recall that an object $X$ of a triangulated category $T$ with infinite direct sums is called compact if the functor $\operatorname{Hom}_{T}(X,-)$ preserves infinite direct sums. A set of compact objects $S \subset T$ generates $T$ as a triangulated category with infinite direct sums if and only if the vanishing of all morphisms $X \longrightarrow Y[*]$ in $T$ for all $X \in S$ implies the vanishing of an object $Y \in T$ [9, Theorem 2.1(2)].

Theorem A. (a) If the $\Gamma$-graded category $B^{\#}$ has finite weak homological dimension and the image of the functor $H^{0}\left(\bmod _{\mathrm{fgp}}^{\mathrm{cdg}}-B\right) \longrightarrow \mathrm{D}^{\mathrm{co}}\left(\bmod ^{\mathrm{cdg}}-B\right)$ generates $\mathrm{D}^{\mathrm{co}}\left(\bmod ^{\mathrm{cdg}}-B\right)$ as a triangulated category with infinite direct sums, then for any right $D G$-module $N_{C}$ and left $D G$-module $M_{C}$ over $C$ the natural morphism $\operatorname{Tor}^{C}\left(N_{C}, M_{C}\right) \longrightarrow \operatorname{Tor}^{C, I I}\left(N_{C}, M_{C}\right)$ is an isomorphism.

The same conclusion holds if the $\Gamma$-graded category $B^{\#}$ has finite weak homological dimension and all objects of $H^{0}\left(\bmod _{\mathrm{fl}}^{\mathrm{cdg}}-B\right)$ can be obtained from objects of $H^{0}\left(\bmod _{\mathrm{fgp}}^{\mathrm{cdg}}-B\right)$ using the operations of shift, cone, filtered inductive limit, and passage to a homotopy equivalent CDG-module over $B$.

(b) If the $\Gamma$-graded category $B^{\#}$ has finite left homological dimension and the image of the functor $H^{0}\left(B-\bmod _{\mathrm{fgp}}^{\mathrm{cdg}}\right) \longrightarrow \mathrm{D}^{\mathrm{co}}\left(B\right.$-mod $\left.{ }^{\mathrm{cdg}}\right)$ generates $\mathrm{D}^{\mathrm{co}}\left(B\right.$-mod $\left.{ }^{\mathrm{cdg}}\right)$ as a triangulated category with infinite direct sums, then for any left DG-modules $L_{C}$ and $M_{C}$ over $C$ the natural morphism $\operatorname{Ext}_{C}^{I I}\left(L_{C}, M_{C}\right) \longrightarrow \operatorname{Ext}_{C}\left(L_{C}, M_{C}\right)$ is an isomorphism.

Proof. The proof is based on the results of Section 3.4. The DG-category $B$ - $\bmod _{\mathrm{fgp}}^{\mathrm{cdg}}$ is equivalent to the DG-category $C^{\mathrm{op}}$. The equivalence assigns to a right CDGmodule $F$ the left CDG-module $G=\operatorname{Hom}^{B^{\text {op }}}(F, B)$ and to a left CDG-module $B$ the right CDG-module $F=\operatorname{Hom}^{B}(G, B)$ over $B$. Given a left CDG-module $M$ over $B$, the corresponding left DG-module $M_{C}$ over $C$ assigns to a CDG-module $F \in \bmod _{\mathrm{fgp}}^{\mathrm{cdg}}-B$ the complex of $k$-modules $F \otimes_{B} M \simeq \operatorname{Hom}^{B}(G, M)$. Given a right CDG-module $N$ over $B$, the corresponding right DG-module $N_{C}$ over $C$ assigns to a CDG-module $F \in \bmod _{\mathrm{fgp}}^{\mathrm{cdg}}-B$ the complex of $k$-modules $\operatorname{Hom}^{B^{\mathrm{op}}}(F, N) \simeq N \otimes_{B} G$.

The categories of (left or right) $\Gamma$-graded modules over the $\Gamma$-graded categories $B^{\#}$ and $C^{\#}$ are also equivalent. $\Gamma$-graded modules corresponding to each other under these equivalences have equal flat, projective, and injective dimensions. So the (weak, left, or right) homological dimensions of the $\Gamma$-graded categories $B^{\#}$ and 
$C^{\#}$ are equal. The equivalence between the DG-categories of (left or right) CDGmodules over $B$ and DG-modules over $C$ preserves the classes of coacyclic, acyclic, and absolutely acyclic (C)DG-modules. Given a left CDG-module $M$ over $B$, the DG-module $M_{C}$ is acyclic if and only if the complex $F \otimes_{B} M \simeq \operatorname{Hom}^{B}(G, M)$ is acyclic for any CDG-modules $F \in \bmod _{\mathrm{fgp}}^{\mathrm{cdg}}-B$ and $G \in B$-mod $\operatorname{fg}_{\mathrm{fgp}}^{\mathrm{cdg}}$ (related to each other as above); similarly for a right CDG-module $N$ over $B$.

For any small CDG-category $B$ the functor $H^{0}\left(B-\bmod _{\text {fgp }}^{\text {cdg }}\right) \longrightarrow \mathrm{D}^{\mathrm{co}}\left(B-\bmod ^{\mathrm{cdg}}\right)$ is fully faithful, and the objects in its image are compact in the coderived category [7]. Thus, the classes of acyclic and coacyclic left DG-modules over $C$ coincide if and only if $\mathrm{D}^{\mathrm{co}}\left(B-\mathrm{mod}^{\mathrm{cdg}}\right)$ is generated by $H^{0}\left(B-\bmod _{\mathrm{fgp}}^{\mathrm{cdg}}\right)$ as a triangulated category with infinite direct sums. Now parts (a) and (b) follow from Proposition 3.4(a)-(b); see also the concluding remarks in Section 3.4. For details related to the proof of the second assertion of part (a), see the last paragraph of the proof of Theorem B below.

The next, more technical result is a generalization of Theorem A to the case of $\Gamma$-graded categories $B^{\#}$ of infinite homological dimension.

Let us denote by $\left\langle T_{i}\right\rangle_{\oplus} \subset T$ (resp., $\left\langle T_{i}\right\rangle_{\sqcap} \subset T$ ) the minimal triangulated subcategory of a triangulated category $T$ containing subcategories $T_{i}$ and closed under infinite direct sums (resp., infinite products). Given a class of CDG-modules $E \subset Z^{0}\left(\bmod ^{\mathrm{cdg}}-B\right)$, we denote by $\langle E\rangle_{\cup} \subset Z^{0}\left(\bmod ^{\mathrm{cdg}}-B\right)$ the full subcategory of all CDG-modules that can be obtained from the objects of $E$ using the operations of shift, cone, filtered inductive limit, and passage to a homotopy equivalent CDG-module.

Theorem B. (a) If for a right $C D G$-module $N \in H^{0}\left(\bmod _{\mathrm{ffd}}^{\mathrm{cdg}}-B\right)$ there exist a right $C D G$-module

$$
Q \in Z^{0}\left(\bmod _{\mathrm{fl}}^{\mathrm{cdg}}-B\right) \cap\left\langle Z^{0}\left(\bmod _{\mathrm{fgp}}^{\mathrm{cdg}}-B\right)\right\rangle_{\cup}
$$

and a closed morphism $Q \longrightarrow N$ with a cone in $\mathrm{Ac}^{\mathrm{co}}\left(\bmod _{\mathrm{ffd}}^{\mathrm{cdg}}-B\right)$, then for any left $D G$-module $M_{C}$ over $C$ the natural morphism $\operatorname{Tor}^{C}\left(N_{C}, M_{C}\right) \longrightarrow \operatorname{Tor}^{C, I I}\left(N_{C}, M_{C}\right)$ is an isomorphism.

(b) If a left $C D G$-module $L \in H^{0}\left(B\right.$ - $\left.\bmod _{\mathrm{fpd}}^{\mathrm{cdg}}\right)$ belongs to

$$
\left\langle H^{0}\left(B-\bmod _{\mathrm{fgp}}^{\mathrm{cdg}}\right), \mathrm{Ac}^{\mathrm{co}}\left(B-\bmod _{\mathrm{fpd}}^{\mathrm{cdg}}\right)\right\rangle_{\oplus} \subset H^{0}\left(B-\bmod _{\mathrm{fpd}}^{\mathrm{cdg}}\right),
$$

then for any left DG-module $M_{C}$ over $C$ the natural morphism $\operatorname{Ext}_{C}^{I I}\left(L_{C}, M_{C}\right) \longrightarrow$ $\operatorname{Ext}_{C}\left(L_{C}, M_{C}\right)$ is an isomorphism.

Equivalently, the same conclusion holds if an object $L \in \mathrm{D}^{\mathrm{co}}\left(B-\bmod _{\mathrm{fpd}}^{\mathrm{cdg}}\right)$ belongs to the minimal triangulated subcategory of $\mathrm{D}^{\mathrm{co}}\left(B-\bmod _{\mathrm{fpd}}^{\mathrm{cdg}}\right)$ containing the image of $H^{0}\left(B-\bmod _{\mathrm{fgp}}^{\mathrm{cdg}}\right)$ and closed under infinite direct sums.

(c) If a left $C D G$-module $M \in H^{0}\left(B\right.$ - $\left.\bmod _{\mathrm{fid}}^{\mathrm{cdg}}\right)$ belongs to

$$
\left\langle\left\{\operatorname{Hom}_{k}\left(F, k^{\vee}\right)\right\}, \operatorname{Ac}^{\mathrm{ctr}}\left(B-\bmod _{\mathrm{fid}}^{\mathrm{cdg}}\right)\right\rangle_{\sqcap} \subset H^{0}\left(B-\bmod _{\mathrm{fid}}^{\mathrm{cdg}}\right), \quad F \in H^{0}\left(\bmod _{\mathrm{fgp}}^{\mathrm{cdg}}-B\right),
$$

then for any left DG-module $L_{C}$ over $C$ the natural morphism $\operatorname{Ext}_{C}^{I I}\left(L_{C}, M_{C}\right) \longrightarrow$ $\operatorname{Ext}_{C}\left(L_{C}, M_{C}\right)$ is an isomorphism.

Equivalently, the same conclusion holds if an object $M \in \mathrm{D}^{\mathrm{ctr}}\left(B-\bmod _{\mathrm{fid}}^{\mathrm{cdg}}\right)$ belongs to the minimal triangulated subcategory of $\mathrm{D}^{\mathrm{ctr}}\left(B-\bmod ^{\mathrm{cdg}}\right)_{\mathrm{fid}}$ containing the objects $\operatorname{Hom}_{k}\left(F, k^{\vee}\right)$, where $F \in H^{0}\left(\bmod _{\mathrm{fgp}}^{\mathrm{cdg}}-B\right)$, and closed under infinite products. 
Proof. The parts (a)-(c) follow from the corresponding parts of Proposition 3.4.

Indeed, a DG-module over any small DG-category $C$ is h-projective if and only if it belongs to the minimal triangulated subcategory of $H^{0}\left(C-\bmod ^{\mathrm{dg}}\right)$ containing the representable DG-modules and closed under infinite direct sums [6, 15]. Representable left DG-modules over $C$ correspond to the objects of $B$ - $\bmod _{\text {fgp }}^{\text {cdg }}$ under the equivalence between the DG-categories $C$-mod ${ }^{\mathrm{dg}}$ and $B$-mod ${ }^{\mathrm{cdg}}$ (see the proof of Theorem A). It follows that the DG-module $L_{C} \in H^{0}\left(C-\bmod _{\mathrm{fpd}}^{\mathrm{dg}}\right)$ belongs to the triangulated subcategory generated by $H^{0}\left(C-\bmod _{\mathrm{fpd}}^{\mathrm{dg}}\right)_{\mathrm{fpd}}$ and the objects coacyclic with respect to $C$ - $\bmod _{\mathrm{fpd}}^{\mathrm{dg}}$ if and only if a CDG-module $L$ over $B$ belongs to the minimal triangulated subcategory of $H^{0}\left(B-\bmod _{\mathrm{fpd}}^{\mathrm{cdg}}\right)$ containing $H^{0}\left(B-\bmod _{\mathrm{fgp}}^{\mathrm{cdg}}\right)$ and all objects coacyclic with respect to $B$ - $\bmod _{\mathrm{fpd}}^{\mathrm{cdg}}$ and closed under infinite direct sums.

Similarly, a left DG-module over a $k$-linear DG-category $C$ is h-injective if and only if it belongs to the minimal triangulated subcategory of $H^{0}\left(C\right.$-mod $\left.{ }^{\mathrm{dg}}\right)$ containing the DG-modules $\operatorname{Hom}_{k}\left(R_{X}, k^{\vee}\right)$, where $R_{X}$ are the representable right DG-modules over $C$. Representable right DG-modules over $C$ correspond to the objects of $\bmod _{\mathrm{fgp}}^{\mathrm{cdg}}-B$ under the equivalence between the DG-categories $\bmod ^{\mathrm{dg}}-C$ and $\bmod ^{\mathrm{cdg}}-B$. So the DG-module $M_{C} \in H^{0}\left(C\right.$-mod $\left.\operatorname{mid}_{\text {fid }}^{\mathrm{dg}}\right)$ belongs to the subcategory generated by $H^{0}\left(C-\bmod _{\text {fid }}^{\mathrm{dg}}\right)_{\text {fid }}$ and the objects contraacyclic with respect to $C$-mod $\operatorname{fid}_{\text {did }}$ if and only if a CDG-module $M$ over $B$ belongs to the minimal triangulated subcategory of $H^{0}\left(B-\bmod _{\text {fid }}^{\text {cdg }}\right)$ containing $A c^{\text {ctr }}\left(B-\bmod _{\text {fid }}^{\text {cdg }}\right)$ and all CDGmodules $\operatorname{Hom}_{k}\left(F, k^{\vee}\right)$ for $F \in H^{0}\left(\bmod _{\mathrm{fgp}}^{\mathrm{cdg}}-B\right)$, and closed inder infinite products.

Finally, a right DG-module over a DG-category $C$ is h-flat whenever it can be obtained from the representable right DG-modules using the operations of shift, cone, filtered inductive limit, and passage to a homotopy equivalent DG-module (we do not know whether the converse is true). Indeed, the class of h-flat DG-modules is closed under shifts, cones, filtered inductive limits, and homotopy equivalences, since these operations commute with the tensor product of DG-modules over $C$ and preserve acyclicity of complexes of $k$-modules. Thus, if a right CDG-module $Q$ over $B$ can be obtained from objects of $\bmod _{\mathrm{fgp}}^{\mathrm{cdg}}-B$ using the operations of shift, cone, filtered inductive limit, and passage to a homotopy equivalent CDG-module, then the corresponding DG-module $Q_{C}$ over $C$ is h-flat.

The equivalence of the two conditions both in (b) and in (c) follows from the same semiorthogonality arguments as in the proof of Proposition 3.4 .

Now assume that the commutative ring $k$ has finite weak homological dimension and the $\Gamma$-graded $k$-modules $B^{\#}(X, Y)$ are flat for all objects $X, Y \in B$. Recall that the DG-categories of left and right CDG-modules over $B \otimes_{k} B^{\mathrm{op}}$ are naturally isomorphic. To any left CDG-module $L$ and right CDG-module $N$ over $B$ one can assign the (left) CDG-module $L \otimes_{k} N$ over the CDG-category $B \otimes_{k} B^{\text {op }}$.

Theorem C. (a) If the $\Gamma$-graded category $B^{\#} \otimes_{k} B^{\# \text { op }}$ has finite weak homological dimension and the image of the functor of tensor product

$$
\otimes_{k}: H^{0}\left(B-\bmod _{\mathrm{fgp}}^{\mathrm{cdg}}\right) \times H^{0}\left(\bmod _{\mathrm{fgp}}^{\mathrm{cdg}}-B\right) \longrightarrow \mathrm{D}^{\mathrm{co}}\left(B \otimes_{k} B^{\mathrm{op}}-\bmod ^{\mathrm{cdg}}\right)
$$

generates $\mathrm{D}^{\mathrm{co}}\left(B \otimes_{k} B^{\mathrm{op}}-\bmod ^{\mathrm{cdg}}\right)$ as a triangulated category with infinite direct sums, then the natural map $H H_{*}\left(C, M_{C}\right) \longrightarrow H H_{*}^{I I}\left(C, M_{C}\right)$ is an isomorphism for any $D G$-module $M_{C}$ over the $D G$-category $C \otimes_{k} C^{\text {op }}$. 
The same conclusion holds if the $\Gamma$-graded category $B^{\#} \otimes_{k} B^{\# \text { op }}$ has finite weak homological dimension and all objects of $H^{0}\left(B \otimes_{k} B^{\mathrm{op}}-\bmod _{\mathrm{fl}}^{\mathrm{cdg}}\right)$ can be obtained from objects in the image of (52) using the operations of shift, cone, filtered inductive limit, and passage to a homotopy equivalent $C D G$-module over $B \otimes_{k} B^{\mathrm{op}}$.

(b) If the $\Gamma$-graded category $B^{\#} \otimes_{k} B^{\# \text { op }}$ has finite left homological dimension and the image of the functor (52) generates $\mathrm{D}^{\mathrm{co}}\left(B \otimes_{k} B^{\mathrm{op}}\right.$-mod $\left.{ }^{\mathrm{cdg}}\right)$ as a triangulated category with infinite direct sums, then the natural map $H^{I I, *}\left(C, M_{C}\right) \longrightarrow$ $H H^{*}\left(C, M_{C}\right)$ is an isomorphism for any $D G$-module $M_{C}$ over the DG-category $C \otimes_{k} C^{\text {op }}$.

Proof. This is a particular case of the next theorem, Theorem D.

Theorem D. (a) Suppose that the $\Gamma$-graded $B^{\#} \otimes_{k} B^{\# \text { op }}$-module $B^{\#}$ has finite flat dimension and there exists a $C D G$-module

$Q \in Z^{0}\left(\bmod _{\mathrm{fl}}^{\mathrm{cdg}}-B \otimes_{k} B^{\mathrm{op}}\right) \cap\left\langle\left\{F \otimes_{k} G\right\}\right\rangle_{\cup}, \quad F \in H^{0}\left(\bmod _{\mathrm{fgp}}^{\mathrm{cdg}}-B\right), G \in H^{0}\left(B-\bmod _{\mathrm{fgp}}^{\mathrm{cdg}}\right)$, and a closed morphism $Q \longrightarrow B$ of $C D G$-modules over $B \otimes_{k} B^{\text {op }}$ with a cone in $\mathrm{Ac}^{\mathrm{co}}\left(\bmod _{\mathrm{ffd}}^{\mathrm{cdg}}-B \otimes_{k} B^{\mathrm{op}}\right)$. Then the natural map $H H_{*}\left(C, M_{C}\right) \longrightarrow H H_{*}^{I I}\left(C, M_{C}\right)$ is an isomorphism for any $D G$-module $M_{C}$ over $C \otimes_{k} C^{\mathrm{op}}$.

(b) Suppose that the $\Gamma$-graded $B^{\#} \otimes_{k} B^{\# \text { op }}$-module $B^{\#}$ has finite projective dimension and the $C D G$-module $B$ over $B \otimes_{k} B^{\text {op }}$ belongs to

$\left\langle\left\{G \otimes_{k} F\right\}, \mathrm{Ac}^{\mathrm{co}}\left(B \otimes_{k} B^{\mathrm{op}}-\bmod _{\mathrm{fpd}}^{\mathrm{cdg}}\right)\right\rangle_{\oplus}, \quad F \in H^{0}\left(\bmod _{\mathrm{fgp}}^{\mathrm{cdg}}-B\right), G \in H^{0}\left(B-\bmod _{\mathrm{fgp}}^{\mathrm{cdg}}\right)$.

Then the natural map $H^{I I}, *\left(C, M_{C}\right) \longrightarrow H H^{*}\left(C, M_{C}\right)$ is an isomorphism for any $D G$-module $M_{C}$ over $C \otimes_{k} C^{\text {op }}$.

Equivalently, the same conclusion holds if the $\Gamma$-graded $B^{\#} \otimes_{k} B^{\# \mathrm{op}}$-module $B^{\#}$ has finite projective dimension and the object $B \in \mathrm{D}^{\mathrm{co}}\left(B \otimes_{k} B^{\mathrm{op}}-\bmod _{\mathrm{fpd}}^{\mathrm{cdg}}\right)$ belongs to the minimal triangulated subcategory of $\mathrm{D}^{\mathrm{co}}\left(B \otimes_{k} B^{\mathrm{op}}-\bmod _{\mathrm{fpd}}^{\mathrm{cdg}}\right)$, containing the $C D G$-modules $G \otimes_{k} F$, where $F \in H^{0}\left(\bmod _{\mathrm{fgp}}^{\mathrm{cdg}}-B\right)$ and $G \in H^{0}\left(B-\bmod _{\mathrm{fgp}}^{\mathrm{cdg}}\right)$, and closed under infinite direct sums.

Proof. It suffices to note that CDG-modules $G \otimes F$ over $B \otimes_{k} B^{\text {op }}$ correspond precisely to representable DG-modules over $C \otimes_{k} C^{\text {op }}$ under the equivalence of DGcategories $B \otimes_{k} B^{\mathrm{op}}-\bmod ^{\mathrm{cdg}} \simeq C \otimes_{k} C^{\mathrm{op}}$-mod ${ }^{\mathrm{dg}}$. The rest of the argument is similar to the proof of Theorem B and based on Proposition 3.4(a)-(b).

3.6. Derived tensor product functor. The following discussion is relevant in connection with the role that the external tensor products of CDG-modules play in the above Theorems 3.5. C-D.

Let $k$ be a commutative ring of finite weak homological dimension, and let $B^{\prime}$ and $B^{\prime \prime}$ be $k$-linear CDG-categories such that the $\Gamma$-graded $k$-modules of morphisms in the categories $B^{\prime \#}$ and $B^{\prime \prime \#}$ are flat. Consider the functor of tensor product

$$
\otimes_{k}: H^{0}\left(B^{\prime}-\bmod ^{\text {cdg }}\right) \times H^{0}\left(B^{\prime \prime}-\bmod ^{\text {cdg }}\right) \longrightarrow H^{0}\left(B^{\prime} \otimes_{k} B^{\prime \prime}-\bmod ^{\text {cdg }}\right) .
$$

We would like to construct its left derived functor

$$
\otimes_{k}^{\mathbb{L}}: \mathrm{D}^{\mathrm{co}}\left(B^{\prime}-\bmod ^{\mathrm{cdg}}\right) \times \mathrm{D}^{\mathrm{co}}\left(B^{\prime \prime}-\bmod ^{\mathrm{cdg}}\right) \longrightarrow \mathrm{D}^{\mathrm{co}}\left(B^{\prime} \otimes_{k} B^{\prime \prime}-\bmod ^{\mathrm{cdg}}\right) .
$$

Denote by $B^{\prime}-\bmod _{k-\mathrm{fl}}^{\mathrm{cdg}}$ the DG-category of left CDG-modules $M^{\prime}$ over $B^{\prime}$ for which all the $\Gamma$-graded $k$-modules $M^{\prime \#}(X)$ are flat, and similarly for CDG-modules 
over $B^{\prime \prime}$. Notice that the natural functor from the quotient category of $H^{0}\left(B^{\prime}-\bmod _{k-\mathrm{fl}}^{\mathrm{cdg}}\right)$ by its intersection with $\mathrm{Ac}^{\mathrm{co}}\left(B^{\prime}-\bmod ^{\mathrm{cdg}}\right)$ to the coderived category $\mathrm{D}^{\mathrm{co}}\left(B^{\prime}-\bmod ^{\mathrm{cdg}}\right)$ is an equivalence of triangulated categories. Indeed, the construction of [15, proof of Theorem 3.6] shows that for any left CDG-module $M^{\prime}$ over $B^{\prime}$ there exists a closed morphism $F^{\prime} \longrightarrow M^{\prime}$, where $F^{\prime} \in H^{0}\left(B^{\prime}-\bmod _{k \text {-fl }}^{\text {cdg }}\right)$, with a coacyclic cone. So it remains to use [15, Lemma 3.6].

Restrict the above functor $\otimes_{k}$ to the subcategory $H^{0}\left(B^{\prime}-\bmod _{k-\mathrm{fl}}^{\mathrm{cdg}}\right)$ in the first argument. Clearly, this restriction factors through the coderived category $\mathrm{D}^{\mathrm{co}}\left(B^{\prime \prime}-\bmod ^{\mathrm{cdg}}\right)$ in the second argument. Let us show that it also factors through the coderived category $\mathrm{D}^{\mathrm{co}}\left(B^{\prime}-\bmod ^{\mathrm{cdg}}\right)$ in the first argument (cf. [14, Lemma 2.7]). Indeed, let $M^{\prime}$ be an object of $H^{0}\left(B^{\prime}-\bmod _{k-\mathrm{fl}}^{\mathrm{cdg}}\right) \cap \mathrm{Ac}^{\mathrm{co}}\left(B^{\prime}-\bmod ^{\mathrm{cdg}}\right)$ and $M^{\prime \prime}$ be a left CDG-module over $B^{\prime \prime}$. Choose a CDG-module $F^{\prime \prime} \in H^{0}\left(B^{\prime \prime}-\bmod _{k \text {-fl }}^{\text {cdg }}\right)$ such that there is a closed morphism $F^{\prime \prime} \longrightarrow M^{\prime \prime}$ with a coacyclic cone. Then the CDGmodule $M^{\prime} \otimes_{k} F^{\prime \prime}$ is coacyclic, since $M^{\prime}$ is coacyclic and $F^{\prime \prime}$ is $k$-flat. At the same time, the cone of the morphism $M^{\prime} \otimes_{k} F^{\prime \prime} \longrightarrow M^{\prime} \otimes_{k} M^{\prime \prime}$ is coacyclic, the cone of the morphism $F^{\prime \prime} \longrightarrow M^{\prime \prime}$ is coacyclic and $M^{\prime}$ is $k$-flat. Thus, the CDG-module $M^{\prime} \otimes_{k} M^{\prime \prime}$ is also coacyclic.

We have constructed the desired derived functor $\otimes_{k}^{L}$. Clearly, the same derived functor can be obtained by restricting the functor $\otimes_{k}$ to the subcategory $H^{0}\left(B^{\prime \prime}-\bmod _{k-\mathrm{fl}}^{\mathrm{cdg}}\right)$ in the second argument.

Analogously, one can construct a derived functor

$$
\otimes_{k}^{\mathbb{L}}: \mathrm{D}^{\mathrm{co}}\left(B^{\prime}-\mathrm{mod}_{\mathrm{fpd}}^{\mathrm{cdg}}\right) \times \mathrm{D}^{\mathrm{co}}\left(B^{\prime \prime}-\bmod _{\mathrm{fpd}}^{\mathrm{cdg}}\right) \longrightarrow \mathrm{D}^{\mathrm{co}}\left(B^{\prime} \otimes_{k} B^{\prime \prime}-\bmod _{\mathrm{fpd}}^{\mathrm{cdg}}\right)
$$

or a similar functor with modules of finite projective dimension replaced by those of finite flat dimension. All one has to do is to restrict the functor $\otimes_{k}$ to the homotopy category of CDG-modules whose underlying $\Gamma$-graded modules satisfy both conditions of $k$-flatness and finiteness of the projective dimension over $B^{\prime}$ or $B^{\prime \prime}$.

In these situations one does not even need the condition that the weak homological dimension of $k$ is finite. However, one has to use the fact that the tensor product over $k$ preserves finiteness of projective/flat dimensions, provided that at least one of the $\Gamma$-graded modules being multiplied is $k$-flat.

\section{EXAMPLES}

The purpose of this section is mainly to illustrate the results of Section 3. Examples of DG-categories $C$ for which the two kinds of Hochschild (co)homology are known to coincide are exhibited in Sections 4.14.5. Examples of CDG-algebras $B$ such that the two kinds of Hochschild (co)homology can be shown to coincide for the DG-category of CDG-modules $C=\bmod _{\mathrm{fgp}}^{\mathrm{cdg}}-B$ are considered in Sections 4.64.8. Counterexamples are discussed in Sections 4.9 and 4.10. The Hochschild (co)homology of matrix factorizations are considered in Sections 4.84 .10 .

4.1. DG-category with zero differentials. Let $C$ be a small $k$-linear DG-category such that the differentials in the complexes $C(X, Y)$ vanish for all objects $X$, $Y \in C$. 
Proposition. (a) If $N$ is a right DG-module over $C$ such that the differentials in the complexes $N(X)$ vanish for all objects $X \in C$ and the $\Gamma$-graded $C^{\#}$-module $N^{\#}$ has finite flat dimension, then the natural morphism $\operatorname{Tor}^{C}(N, M) \longrightarrow \operatorname{Tor}^{C, I I}(N, M)$ is an isomorphism for any left $D G$-module $M$ over $C$.

(b) If $L$ is a left $D G$-module over $C$ such that the differentials in the complexes $L(X)$ vanish for all objects $X \in C$ and the $\Gamma$-graded $C^{\#}$-module $L^{\#}$ has finite projective dimension, then the natural morphism $\operatorname{Ext}_{C}^{I I}(L, M) \longrightarrow \operatorname{Ext}_{C}(L, M)$ is an isomorphism for any left $D G$-module $M$ over $C$.

(c) If $M$ is a left DG-module over $C$ such that the differentials in the complexes $M(X)$ vanish for all objects $X \in C$ and the $\Gamma$-graded $C^{\#-m o d u l e ~} M^{\#}$ has finite injective dimension, then the natural morphism $\operatorname{Ext}_{C}^{I I}(L, M) \longrightarrow \operatorname{Ext}_{C}(L, M)$ is an isomorphism for any left $D G$-module $L$ over $C$.

Proof. To prove part (a), notice that a finite flat left resolution $P_{\bullet}$ of the $\Gamma$-graded $C^{\#}$-module $N^{\#}$, with every term of it endowed with a zero differential, can be used to compute both kinds of derived functor Tor in which we are interested. The proofs of parts (b) and (c) are similar.

Corollary A. (a) If the $\Gamma$-graded category $C^{\#}$ has finite weak homological dimension, then the natural morphism $\operatorname{Tor}^{C}(N, M) \longrightarrow \operatorname{Tor}^{C, I I}(N, M)$ is an isomorphism for any $D G$-modules $N$ and $M$.

(b) If the $\Gamma$-graded category $C^{\#}$ has finite left homological dimension, then the natural morphism $\operatorname{Ext}_{C}^{I I}(L, M) \longrightarrow \operatorname{Ext}_{C}(L, M)$ is an isomorphism for any left $D G$-modules $L$ and $M$ over $C$.

Proof. Any DG-module over a DG-category with vanishing differentials is an extension of two DG-modules with vanishing differentials. Indeed, the kernel and image of the differential $d$ on such a DG-module is a DG-submodule. So it remains to use the fact that both kinds of functors Ext and Tor assign distinguished triangles to short exact sequences of DG-modules in any argument, together with the preceding proposition. Part (b) also follows from the fact that the classes of acyclic and absolutely acyclic left DG-modules over $C$ coincide in its assumptions; see 7 .

Corollary B. Let $C$ be a $D G$-category such that the complexes $C(X, Y)$ are complexes of flat $k$-modules with zero differentials for all objects $X, Y \in C$.

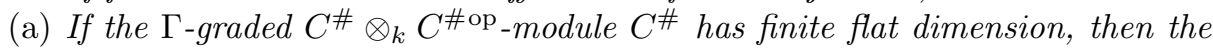
natural morphism of Hochschild homology $H_{*}(C, M) \longrightarrow H H_{*}^{I I}(C, M)$ is an isomorphism for any $D G$-module $M$ over $C \otimes_{k} C^{\text {op }}$.

(b) If the $\Gamma$-graded $C^{\#} \otimes_{k} C^{\# \text { op }}$-module $C^{\#}$ has finite projective dimension, then the natural morphism of Hochschild cohomology $H H^{I I}, *(C, M) \longrightarrow H H^{*}(C, M)$ is an isomorphism for any $D G$-module $M$ over $C \otimes_{k} C^{\text {op }}$.

Proof. This follows directly from the Proposition.

4.2. Nonpositive DG-category. Assume that our grading group $\Gamma$ is isomorphic to $\mathbb{Z}$ and the isomorphism identifies 1 with 1 (see Section 1.1).

Let $C$ be a small $k$-linear DG-category. Assume that the complexes of $k$-modules $C(X, Y)$ are concentrated in nonpositive degrees for all objects $X, Y \in C$. Let us call a (left or right) DG-module $M$ over $C$ bounded above if all the complexes of $k$ modules $M(X)$ are bounded above uniformly, i.e., there exists an integer $n$ such that the complexes $M(X)$ are concentrated in the degree $\leqslant n$ for all $X$. DG-modules bounded below are defined in the similar way. 
Proposition A. (a) If a right DG-module $N$ and a left $D G$-module $M$ over $C$ are bounded above, then the natural morphism

$$
\operatorname{Tor}^{C}(N, M) \longrightarrow \operatorname{Tor}^{C, I I}(N, M)
$$

is an isomorphism.

(b) If a left DG-module $L$ over $C$ is bounded above and a left DG-module $M$ is bounded below, then the natural morphism $\operatorname{Ext}_{C}^{I I}(L, M) \longrightarrow \operatorname{Ext}_{C}(L, M)$ is an isomorphism.

Proof. The proof is based on the construction of the natural morphisms (9)-(10) from Section 2.2. To prove part (a), notice that there exists a left projective resolution $Q$ • of the DG-module $N$ in the exact category $Z^{0}\left(\bmod ^{\mathrm{dg}}-C\right)$ consisting of DG-modules bounded above with the same constant $n$ as the DG-module $N$, and then there is no difference between the two kinds of totalizations of the bicomplex $Q \bullet \otimes_{C} M$. The proof of part (b) is similar.

Proposition B. (a) If a right DG-module $N$ over $C$ is bounded above and the graded $C^{\#}$-module $N^{\#}$ has finite flat dimension, then the natural morphism

$$
\operatorname{Tor}^{C}(N, M) \longrightarrow \operatorname{Tor}^{C, I I}(N, M)
$$

is an isomorphism for any left DG-module $M$ over $C$.

(b) If a left DG-module $L$ over $C$ is bounded above and the graded $C^{\#}$-module $L^{\#}$ has finite projective dimension, then the natural morphism $\operatorname{Ext}_{C}^{I I}(L, M) \longrightarrow$ $\operatorname{Ext}_{C}(L, M)$ is an isomorphism for any left $D G$-module $M$ over $C$.

(c) If a left DG-module $M$ over $C$ is bounded below and the graded $C^{\#}$-module $M^{\#}$ has finite injective dimension, then the natural morphism $\operatorname{Ext}_{C}^{I I}(L, M) \longrightarrow$ $\operatorname{Ext}_{C}(L, M)$ is an isomorphism for any left $D G$-module $L$ over $C$.

Proof. Parts (a)-(c) follow from the corresponding parts of Proposition 3.4.

To prove part (b), let us choose a finite left resolution $P_{\bullet}$ of the DG-module $L$ in the abelian category $Z^{0}\left(C\right.$-mod $\left.{ }^{\mathrm{dg}}\right)$ such that the DG-modules $P_{i}$ are bounded above and their underlying graded $C^{\#}$-modules are projective. Then the total DG-module $P$ of $P$. maps into $L$ with a cone absolutely acyclic with respect to $C$ - $\bmod _{\mathrm{fpd}}^{\mathrm{dg}}$, so it suffices to show that $P$ is h-projective. Indeed, any left DG-module $P$ over $C$ that is bounded above and projective as a graded $C^{\#}$-module is h-projective. To prove the latter assertion, one can construct by induction in $n$ an increasing filtration of $P$ by DG-submodules such that the associated quotient DG-modules are direct summands of direct sums of representable DG-modules shifted by the degree determined by the number of the filtration component.

The proof of part (c) is similar up to duality, and to prove part (a) one has to show that a right DG-module $Q$ over $C$ that is bounded above and flat as a graded $C^{\#}$-module is h-flat. This can be done, e.g., by using (the graded version of) the Govorov-Lazard flat module theorem to construct a filtration similar to the one in the projective case, except that the associated quotient DG-modules are filtered inductive limits of direct sums of (appropriately shifted) representable DG-modules.

Now assume that the complexes $C(X, Y)$ are complexes of flat $k$-modules concentrated in nonpositive cohomological degrees. 
Corollary. (a) For any DG-module $M$ over $C \otimes_{k} C^{\text {op }}$ bounded above, the natural morphism $H_{*}(C, M) \longrightarrow H H_{*}^{I I}(C, M)$ is an isomorphism. If the graded $C^{\#} \otimes_{k} C^{\# \text { op }}$-module $C^{\#}$ has finite flat dimension, then the latter morphism is an isomorphism for any DG-module $M$.

(b) For any $D G$-module $M$ over $C \otimes_{k} C^{\text {op }}$ bounded below, the natural morphism $H H^{I I, *}(C, M) \longrightarrow H H^{*}(C, M)$ is an isomorphism. If the graded $C^{\#} \otimes_{k} C^{\# \text { op }}$ module $C^{\#}$ has finite projective dimension, then the latter morphism is an isomorphism for any $D G$-module $M$.

Proof. Apply Propositions A and B(a)-(b) to the DG-category $C \otimes_{k} C^{\mathrm{op}}$.

So the map $H H_{*}(C) \longrightarrow H H_{*}^{I I}(C)$ is an isomorphism under our assumptions on $C$. The map $H H^{I I, *}(C) \longrightarrow H H^{*}(C)$ is an isomorphism provided that either the DG-module $C$ over $C \otimes_{k} C^{\text {op }}$ is bounded below [1. Proposition 3.15] or the graded $C^{\#} \otimes_{k} C^{\# \text { op}}$-module $C^{\#}$ has finite projective dimension.

4.3. Strongly positive DG-category. As in Section 4.2, we assume that the grading group $\Gamma$ is isomorphic to $\mathbb{Z}$ and the isomorphism identifies $\mathbf{1}$ with 1 .

Let $k$ be a field and $C$ be a $k$-linear DG-category such that the complexes of $k$-vector spaces $C(X, Y)$ are concentrated in nonnegative degrees for all objects $X, Y \in C$, the component $C^{1}(X, Y)$ vanishes for all $X$ and $Y$, the component $C^{0}(X, Y)$ vanishes for all nonisomorphic $X$ and $Y$, and the $k$-algebra $C^{0}(X, X)$ is semisimple for all $X$. Here a noncommutative ring is called (classically) semisimple if the abelian category of (left or right) modules over it is semisimple. We keep the terminology from Section 4.2 related to bounded DG-modules.

Proposition A. (a) If a right DG-module $N$ and a left $D G$-module $M$ over $C$ are bounded below, then the natural morphism $\operatorname{Tor}^{C}(N, M) \longrightarrow \operatorname{Tor}^{C, I I}(N, M)$ is an isomorphism.

(b) If a left DG-module $L$ over $C$ is bounded below and a left DG-module $M$ is bounded above, then the natural morphism $\operatorname{Ext}_{C}^{I I}(L, M) \longrightarrow \operatorname{Ext}_{C}(L, M)$ is an isomorphism.

Proof. The proof uses the construction of the natural morphisms (9)-(10) from Section 2.2. To prove part (a), one can compute both kinds of Tor in question using the reduced bar-resolution of the DG-module $N$ over $C$ relative to $C^{0}$, i. e.,

$\cdots \longrightarrow N \otimes_{C^{0}} C / C^{0} \otimes_{C^{0}} C / C^{0} \otimes_{C^{0}} C \longrightarrow N \otimes_{C^{0}} C / C^{0} \otimes_{C^{0}} C \longrightarrow N \otimes_{C^{0}} C$.

Here $C^{0}$ is considered as a DG-category with complexes of morphisms concentrated in degree 0 and endowed with zero differentials, $C / C_{0}$ is a DG-module over $C^{0} \otimes_{k}$ $C^{0 \text { op }}$, and $C$ is a DG-module over $C^{0} \otimes_{k} C^{\text {op }}$. The semisimplicity condition on $C^{0}$ guarantees projectivity of right DG-modules of the form $R \otimes_{C^{0}} C$ as objects of the exact category $Z^{0}\left(\bmod ^{\mathrm{dg}}-C\right)$ for all right DG-modules $R$ over $C^{0}$. Due to the positivity/boundedness conditions on $C, N$, and $M$, there is no difference between the two kinds of totalizations of the resulting bar-bicomplex. The proof of part (b) is similar.

Proposition B. (a) If a right DG-module $N$ over $C$ is bounded below and the graded $C^{\#-m o d u l e ~} N^{\#}$ has finite flat dimension, then the natural morphism

$$
\operatorname{Tor}^{C}(N, M) \longrightarrow \operatorname{Tor}^{C, I I}(N, M)
$$

is an isomorphism for any left $D G$-module $M$ over $C$. 
(b) If a left DG-module $L$ over $C$ is bounded below and the graded $C^{\#}$-module $L^{\#}$ has finite projective dimension, then the natural morphism $\operatorname{Ext}_{C}^{I I}(L, M) \longrightarrow$ $\operatorname{Ext}_{C}(L, M)$ is an isomorphism for any left DG-module $M$ over $C$.

(c) If a left DG-module $M$ over $C$ is bounded above and the graded $C^{\#-m o d u l e}$ $M^{\#}$ has finite injective dimension, then the morphism $\operatorname{Ext}_{C}^{I I}(L, M) \longrightarrow \operatorname{Ext}_{C}(L, M)$ is an isomorphism for any left DG-module $L$.

Proof. The proof is similar to that of Proposition 4.2.B. E.g., in part (b) the key is to show that any DG-module over $C$ that is bounded below and projective as a graded $C^{\#}$-module is h-projective. One constructs an increasing filtration similar to that in Section 4.2 with the only difference being that the associated quotient DG-modules are projective objects of the exact category $Z^{0}\left(C\right.$-mod $\left.{ }^{\mathrm{dg}}\right)$.

Corollary. (a) For any $D G$-module $M$ over $C \otimes_{k} C^{\text {op }}$ bounded below, the natural morphism $H_{*}(C, M) \longrightarrow H H_{*}^{I I}(C, M)$ is an isomorphism. If the graded $C^{\#} \otimes_{k} C^{\# \text { op }}$-module $C^{\#}$ has finite flat dimension, then the latter morphism is an isomorphism for any $D G$-module $M$.

(b) For any $D G$-module $M$ over $C \otimes_{k} C^{\text {op }}$ bounded above, the natural morphism $H H^{I I, *}(C, M) \longrightarrow H H^{*}(C, M)$ is an isomorphism. If the graded $C^{\#} \otimes_{k} C^{\# \text { op }}$ module $C^{\#}$ has finite projective dimension, then the latter morphism is an isomorphism for any $D G$-module $M$.

So the map $H H_{*}(C) \longrightarrow H H_{*}^{I I}(C)$ is an isomorphism under our assumptions on $C$. The map $H H^{I I}, *(C) \longrightarrow H H^{*}(C)$ is an isomorphism provided that either the DG-module $C$ over $C \otimes_{k} C^{\text {op }}$ is bounded above or the graded $C^{\#} \otimes_{k} C^{\# \text { op }}$ module $C^{\#}$ has finite projective dimension.

4.4. Cofibrant DG-category. A small $k$-linear DG-category is called cofibrant if it is a retract (in the category of DG-categories and functors between them) of a DG-category $k\left\langle x_{n, \alpha}\right\rangle$ of the following form. As a $\Gamma$-graded category, $k\left\langle x_{n, \alpha}\right\rangle$ is freely generated by a set of homogeneous morphisms $x_{n, \alpha}$, where $n$ runs over nonnegative integers and $\alpha$ belongs to some set of indices. This means that the morphisms in $k\left\langle x_{n, \alpha}\right\rangle$ are the formal $k$-linear combinations of formal compositions of the morphisms $x_{n, \alpha}$. It is additionally required that the element $d x_{n, \alpha}$ belongs to the class of morphisms multiplicatively and additively generated by the morphisms $x_{m, \beta}$ with $m<n$. The cofibrant DG-categories are exactly (up to the zero object issue) the cofibrant objects in the model category structure constructed in [20] (see also [21]).

The following lemmas will be used in conjunction with the results of Section 3.4 in order to prove comparison results for the two kinds of Ext, Tor, and Hochschild (co)homology for cofibrant DG-categories.

Lemma A. Let $D$ be a $D G$-category of the form $k\left\langle x_{n, \alpha}\right\rangle$ as above.

(a) If a right $D G$-module $N$ over $D$ is such that all the complexes of $k$-modules $N(X)$ are $h$-flat complexes of flat $k$-modules, then there exists a closed morphism $Q \longrightarrow N$, where $Q \in H^{0}\left(\bmod _{\mathrm{fl}}^{\mathrm{dg}}-D\right)_{\mathrm{fl}}$, with a cone absolutely acyclic with respect to $\bmod _{\mathrm{ffd}}^{\mathrm{dg}}-D$.

(b) If a left $D G$-module $L$ over $D$ is such that all the complexes of $k$-modules $L(X)$ are $h$-projective complexes of projective $k$-modules, then there exists a closed morphism $P \longrightarrow L$, where $L \in H^{0}\left(D \text {-mod } \operatorname{prj}_{\mathrm{prj}}^{\mathrm{dg}}\right)_{\mathrm{prj}}$, with a cone absolutely acyclic with respect to $D$ - $\bmod _{\mathrm{fpd}}^{\mathrm{dg}}$. 
(c) If a left DG-module $M$ over $D$ is such that all the complexes of $k$-modules $L(X)$ are h-injective complexes of injective $k$-modules, then there is a closed morphism $M \longrightarrow J$, where $J \in H^{0}\left(D-\bmod _{\mathrm{inj}}^{\mathrm{dg}}\right)_{\mathrm{inj}}$, with a cone absolutely acyclic with respect to $D$-mod fid $_{\text {dg }}$.

Lemma B. (a) If $C$ is a cofibrant $k$-linear DG-category and the ring $k$ has a finite weak homological dimension, then the weak homological dimension of the $\Gamma$-graded category $C^{\#}$ is also finite. Moreover, the categories $H^{0}\left(\bmod _{\mathrm{fl}}^{\mathrm{dg}}-C\right)$ and $H^{0}\left(\bmod _{\mathrm{fl}}^{\mathrm{dg}}-C\right)_{\mathrm{fl}}$ coincide in this case.

(b) If $C$ is a cofibrant $k$-linear DG-category and the ring $k$ has finite homological dimension, then the left homological dimension of the $\Gamma$-graded category $C^{\#}$ is finite. Moreover, the classes of acyclic and absolutely acyclic left DG-modules over $C$ coincide in this case.

Proof of Lemmas A and B. Let us first prove parts (b) of both lemmas. The following arguments generalize the proof of [15, Theorem 9.4] to the DG-category case. For any objects $X, Y \in D$ denote by $V(X, Y)$ the free $\Gamma$-graded $k$-module spanned by those elements $x_{n, \alpha}$ that belong to $D(X, Y)$. Consider the short exact sequence of $\Gamma$-graded $D^{\#}$-modules

$\bigoplus_{Y, Z \in D} D(X, Y) \otimes_{k} V(Y, Z) \otimes_{k} L(Z) \longrightarrow \bigoplus_{Y \in D} D(X, Y) \otimes_{k} L(Y) \longrightarrow L(X)$

The middle and right terms are endowed with DG-module structures, so the left term also acquires such a structure. There is a natural increasing filtration on the left term induced by the filtration of $V$ related to the indexes $n$ of the generators $x_{n, \alpha}$. It is a filtration by DG-submodules, and the differentials on the associated quotient modules are the differentials on the tensor product induced by the differentials on the factors $D$ and $L$ (as is the differential on the middle term).

It follows that whenever all the complexes of $k$-modules $L(X)$ are coacyclic (absolutely acyclic), both the middle and the left terms of the exact sequence are coacyclic (absolutely acyclic) DG-modules, so $L(X)$ is also a coacyclic (absolutely acyclic) DG-module. In particular, if the homological dimension of $k$ is finite and $L$ is acyclic, then it is absolutely acyclic. Furthermore, when all the complexes $L(X)$ are h-projective complexes of projective $k$-modules, both the middle and the left terms belong to $H^{0}\left(D-\bmod _{\mathrm{prj}}^{\mathrm{dg}}\right)_{\mathrm{prj}}$. So it suffices to take the cone of the left arrow as the DG-module $P$.

It also follows from the same exact sequence considered as an exact sequence of $\Gamma$ graded $D^{\#}$-modules that the $\Gamma$-graded $D^{\#}$-module $L^{\#}$ has the projective dimension at most 1 whenever all $L^{\#}(X)$ are projective $\Gamma$-graded $k$-modules. Since for any projective $\Gamma$-graded $D^{\#}$-module $F^{\#}$ the $\Gamma$-graded $k$-modules $F^{\#}(X)$ are projective, the left homological dimension of $D^{\#}$ can exceed the homological dimension of $k$ by at most 1 .

Since Ext and Tor over $\Gamma$-graded categories are functorial with respect to $\Gamma$ graded functors, the (weak, left, or right) homological dimension of a retract $C^{\#}$ of a $\Gamma$-graded category $D^{\#}$ does not exceed that of $D^{\#}$. To prove the second assertion of Lemma $\mathrm{B}(\mathrm{b})$ for a retract $C$ of a DG-category $D$ as above, consider DG-functors $I: C \longrightarrow D$ and $\Pi: D \longrightarrow C$ such that $\Pi I=\operatorname{Id}_{C}$. Let $M$ be an acyclic DG-module 
over $C$. Then the DG-module $\Pi^{*} M$ over $D$ is acyclic, hence absolutely acyclic, and it follows that $M=I^{*} \Pi^{*} M$ is also absolutely acyclic.

It remains to prove the second assertion of Lemma $\mathrm{B}(\mathrm{a})$. If the underlying $\Gamma$ graded $D^{\#}$-module of a right DG-module $N$ over $D$ is flat, then the above exact sequence remains exact after taking the tensor product with $N$ over $D$. Besides, the $\Gamma$-graded $k$-modules $N^{\#}(X)$ are flat since the $\Gamma$-graded $k$-modules $D^{\#}(X, Y)$ are. If the weak homological dimension of $k$ is finite, it follows that the complexes of $k$-modules $N(X)$ are h-flat. Now if the complexes of $k$-modules $L(X)$ are acyclic, then the tensor products of the left and the middle terms with $N$ over $D$ are acyclic, hence the complex $N \otimes_{D} L$ is also acyclic.

Finally, let us deduce the same assertion for a retract $C$ of the DG-category $D$. For this purpose, notice that for any DG-functor $F: C \longrightarrow D$ the functor

$$
F^{*}: H^{0}\left(\bmod ^{\mathrm{dg}}-D\right) \longrightarrow H^{0}\left(\bmod ^{\mathrm{dg}}-C\right)
$$

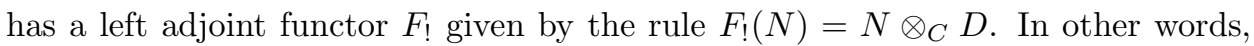
the DG-module $F_{!}(N)$ assigns the complex of $k$-modules $N \otimes_{C} F^{*} S_{X}$ to an object $X \in D$, where $S_{X}$ is the left (covariant) representable DG-module over $D$ corresponding to $X$. The functor $F_{\text {! }}$ transforms objects of $H^{0}\left(\bmod _{\mathrm{fl}}^{\mathrm{dg}}-C\right)$ to objects of $H^{0}\left(\bmod _{\mathrm{fl}}^{\mathrm{dg}}-D\right)$ and h-flat DG-modules to h-flat DG-modules, since for any right DGmodule $N$ over $C$ and left DG-module $M$ over $D$ one has $F_{!} N \otimes_{D} M \simeq N \otimes_{C} F^{*} M$. Now if $(I, \Pi)$ is our retraction and $N \in H^{0}\left(\bmod _{\mathrm{fl}}^{\mathrm{dg}}-C\right)$, then $I_{!} N \in H^{0}\left(\bmod _{\mathrm{fl}}^{\mathrm{dg}}-D\right)$, hence $I_{!} N$ is h-flat, and it follows that $N=\Pi_{!} I_{!} N$ is also h-flat.

Corollary C. Let $C$ be a cofibrant $k$-linear DG-category.

(a) Given a right $D G$-module $N$ and a left $D G$-module $M$ over $C$, the natural morphism $\operatorname{Tor}^{C}(N, M) \longrightarrow \operatorname{Tor}^{C, I I}(N, M)$ is an isomorphism provided that either all the complexes $N(X)$ or all the complexes $M(X)$ are $h$-flat complexes of flat $k$ modules. When the ring $k$ has finite weak homological dimension, this morphism is an isomorphism for any $D G$-modules $N$ and $M$.

(b) Given two left $D G$-modules $L$ and $M$ over $C$, the natural morphism

$$
\operatorname{Ext}_{C}^{I I}(L, M) \longrightarrow \operatorname{Ext}_{C}(L, M)
$$

is an isomorphism provided that either all the complexes $L(X)$ are h-projective complexes of projective $k$-modules or all the complexes $M(X)$ are h-injective complexes of injective $k$-modules. When the ring $k$ has finite homological dimension, this morphism is an isomorphism for any DG-modules $L$ and $M$.

Proof. Since the morphisms (9)-(10) are functorial with respect to DG-functors $F: C \longrightarrow D$, i.e., make commutative squares with the morphisms (3)-(4) and (17) -(8), it suffices to prove the statements of Corollary C for a DG-category $D=$ $k\left\langle x_{n, \alpha}\right\rangle$. Now the first assertions in both (a) and (b) follow from Lemma A and Proposition 3.4, while the second ones follow from Lemma B and the concluding remarks in Section 3.4 .

Lemma D. Let $D$ be a DG-category of the form $k\left\langle x_{n, \alpha}\right\rangle$. Then the $\Gamma$-graded $D^{\#} \otimes_{k} D^{\# \text { op }}$-module $D^{\#}$ has projective dimension at most 1 . There exists an $h$ projective $D G$-module $P$ over $D \otimes_{k} D^{\text {op }}$ and a closed morphism of DG-modules $P \longrightarrow D$ with a cone absolutely acyclic with respect to $D \otimes_{k} D^{\mathrm{op}}-\bmod _{\mathrm{fpd}}^{\mathrm{dg}}$. 
Proof. It suffices to consider the short exact sequence

$$
\begin{aligned}
\bigoplus_{Y^{\prime}, Y^{\prime \prime} \in D} D\left(X, Y^{\prime}\right) \otimes_{k} V\left(Y^{\prime}, Y^{\prime \prime}\right) & \otimes_{k} D\left(Y^{\prime \prime}, Z\right) \\
& \longrightarrow \bigoplus_{Y \in D} D(X, Y) \otimes_{k} D(Y, Z) \longrightarrow D(X, Z)
\end{aligned}
$$

and argue as above.

Corollary E. Let $C$ be a cofibrant k-linear DG-category. Then for any DGmodule $M$ over $C \otimes_{k} C^{\text {op }}$, the natural morphisms of Hochschild (co)homology $H H_{*}(C, M) \longrightarrow H H_{*}^{I I}(C, M)$ and $H H^{I I, *}(C, M) \longrightarrow H H^{*}(C, M)$ are isomorphisms.

Proof. The assertions for a DG-category $D=k\left\langle x_{n, \alpha}\right\rangle$ follow from Lemma D and Proposition 3.4(a)-(b). To deduce the same results for a retract $C$ of a DG-category $D$, use the fact that the comparison morphisms (23) make commutative squares with the morphisms (16), (19) and (20), (22).

4.5. DG-algebra with Koszul filtration. Let $A$ be a DG-algebra over a field $k$ endowed with an increasing filtration $F_{i} A, i \geqslant 0$, such that $F_{0} A=k, F_{i} A \cdot F_{j} A \subset$ $F_{i+j} A$, and $d F_{i} A \subset F_{i+1} A$. Assume that the associated graded algebra $\operatorname{gr}_{F} A$ is Koszul (in the grading $i$ induced by the filtration $F$ ) and has finite homological dimension (here we use the Koszulity condition without the assumption of finitedimensionality of the components of $\operatorname{gr}_{F} A$; see e.g. 17]). Then one can assign to $A$ a coaugmented CDG-coalgebra $\mathcal{C}$ endowed with a finite decreasing filtration $G$ [15, Section 6.8] (cf. Section 4.6 below).

Corollary. Assume that the coaugmented coalgebra $\mathrm{C}$ is conilpotent (see [17] or [15. Section 6.4]). Then for any right DG-module $N$ and left $D G$-module $M$ over $A$ the natural morphism $\operatorname{Tor}^{A}(N, M) \longrightarrow \operatorname{Tor}^{A, I I}(N, M)$ is an isomorphism. For any left $D G$-modules $L$ and $M$ over $A$ the natural morphism $\operatorname{Ext}_{A}^{I I}(L, M) \longrightarrow \operatorname{Ext}_{A}(L, M)$ is an isomorphism. For any DG-module $M$ over $A \otimes_{k} A^{\text {op }}$ the natural maps of Hochschild (co)homology $H H_{*}(A, M) \longrightarrow H H_{*}^{I I}(A, M)$ and $H H^{I I, *}(A, M) \longrightarrow$ $H H^{*}(A, M)$ are isomorphisms.

Proof. The (left or right) homological dimension of the graded algebra $A^{\#}$ is finite, since one can compute the spaces Ext over it using the nonhomogeneous Koszul resolution. By [15, Corollary 6.8.2], the classes of acyclic and absolutely acyclic DGmodules over $A$ coincide. Hence the first two assertions follow from the concluding remarks in Section 3.4. To prove the last assertion, notice that the DG-algebra $A \otimes_{k} A^{\text {op }}$ is endowed with the induced filtration having the same properties as required above for the filtration on $A$; the corresponding CDG-coalgebra is naturally identified with $\mathcal{C} \otimes_{k} \mathcal{C o p}$. Since $\mathcal{C}$ is conilpotent, so is $\mathcal{C} \otimes_{k} \mathcal{C}^{o p}$. Thus, the classes of acyclic and absolutely acyclic DG-modules over $A \otimes_{k} A^{\text {op }}$ coincide, too.

4.6. CDG-algebra with Koszul filtration. Let $B=(B, d, h)$ be a CDG-algebra over a field $k$ endowed with an increasing filtration $F_{i} B, i \geqslant 0$, such that $F_{0} B=k$, $F_{i} B \cdot F_{j} B \subset F_{i+j} B, d F_{i} B \subset F_{i+1} B$, and $h \in F_{2} B$. Assume that the associated graded algebra $\operatorname{gr}_{F} B$ is Koszul and has finite homological dimension. Then one can assign to the filtered CDG-algebra $(B, F)$ a $C D G$-coalgebra $\mathcal{C}$ endowed with a finite decreasing filtration $G$ [15, Section 6.8]. 
Let $C=\bmod _{\mathrm{fgp}}^{\mathrm{cdg}}-B$ be the DG-category of right CDG-modules over $B$, projective and finitely generated as $\Gamma$-graded $B^{\#}$-modules. All the results below will also hold for finitely generated free modules in place of finitely generated projective ones.

Corollary A. For any right $D G$-module $N$ and left $D G$-module $M$ over $C$ the natural map $\operatorname{Tor}^{C}(N, M) \longrightarrow \operatorname{Tor}^{C, I I}(N, M)$ is an isomorphism. For any left $D G$ modules $L$ and $M$ over $C$ the natural map $\operatorname{Ext}_{C}^{I I}(L, M) \longrightarrow \operatorname{Ext}_{C}(L, M)$ is an isomorphism.

Proof. The homological dimension of the graded algebra $B^{\#}$ is finite (see Section 4.5). By [15, Corollary 6.8.1], the coderived category $\mathrm{D}^{\mathrm{co}}\left(B\right.$-mod $\left.{ }^{\mathrm{cdg}}\right)$ is generated by $H^{0}\left(B-\bmod _{\mathrm{fgp}}^{\mathrm{cdg}}\right)$ as a triangulated category with infinite direct sums. Thus, the assertions of the corollary follow from Theorem 3.5. A.

Let $\mathcal{C}^{\text {ss }}$ denote the maximal cosemisimple $\Gamma$-graded subcoalgebra of the $\Gamma$-graded coalgebra $\mathcal{C}$ [15, Section 5.5]. Assume that the differential $d$ and the curvature linear function $h$ on $\mathcal{C}$ annihilate $C^{\mathrm{ss}}$ and that the tensor product coalgebra $\mathcal{C}^{\mathrm{ss}} \otimes_{k} \mathrm{C}^{\mathrm{ss}}$ op is cosemisimple. The latter condition always holds when the field $k$ is perfect and the grading group $\Gamma$ contains no torsion of the order equal to the characteristic of $k$.

Corollary B. Under the above assumptions, the natural maps of Hochschild (co)homology $H_{*}(C, M) \longrightarrow H H_{*}^{I I}(C, M)$ and $H H^{I I, *}(C, M) \longrightarrow H H^{*}(C, M)$ are isomorphisms for any $D G$-module $M$ over the $D G$-category $C \otimes_{k} C^{\mathrm{op}}$.

Proof. The CDG-algebra $B \otimes_{k} B^{\text {op }}$ is endowed with the induced filtration having the same properties; the corresponding CDG-coalgebra is naturally identified with $\mathcal{C} \otimes_{k} \mathcal{C}^{\text {op }}$. The coderived category of CDG-modules $\mathrm{D}^{\mathrm{co}}\left(B-\bmod ^{\mathrm{cdg}}\right)$ is equivalent to the coderived category of CDG-comodules $\mathrm{D}^{\mathrm{co}}\left(\mathcal{C}_{-}-\mathrm{comod}^{\mathrm{cdg}}\right)$ [15, Theorem 6.8]. This equivalence transforms the functor of tensor product

$$
\otimes_{k}: \mathrm{D}^{\mathrm{co}}\left(B-\mathrm{mod}^{\mathrm{cdg}}\right) \times \mathrm{D}^{\mathrm{co}}\left(B^{\mathrm{op}}-\bmod ^{\mathrm{cdg}}\right) \longrightarrow \mathrm{D}^{\mathrm{co}}\left(B \otimes_{k} B^{\mathrm{op}}-\bmod ^{\mathrm{cdg}}\right)
$$

into the similar functor of tensor product

$$
\otimes_{k}: \mathrm{D}^{\mathrm{co}}\left(\mathcal{C}_{-} \operatorname{comod}^{\mathrm{cdg}}\right) \times \mathrm{D}^{\mathrm{co}}\left(\mathcal{C}^{\mathrm{op}}-\operatorname{comod}^{\mathrm{cdg}}\right) \longrightarrow \mathrm{D}^{\mathrm{co}}\left(\mathcal{C} \otimes_{k} \mathrm{e}^{\mathrm{op}}-\operatorname{comod}^{\mathrm{cdg}}\right) .
$$

When the coalgebra $\mathcal{C}^{\mathrm{ss}} \otimes_{k} \mathrm{e}^{\mathrm{ss} \text { op }}$ is cosemisimple, any DG-comodule over it (considered as a DG-coalgebra with zero differential) can be obtained from tensor products of DG-comodules over $\mathcal{C}^{\text {ss }}$ and $\mathcal{C}^{\text {ssop }}$ using the operations of cone and passage to a direct summand. The coderived category $\mathrm{D}^{\mathrm{co}}\left(\mathcal{C} \otimes_{k} \mathrm{Cop}_{-\mathrm{comod}^{\mathrm{cdg}}}\right)$ of CDG-comodules over $\mathcal{C} \otimes_{k} \mathcal{C}^{\text {op }}$ is generated by DG-comodules over $\mathcal{C}^{\mathrm{ss}} \otimes_{k} \mathcal{C}^{\text {ss op }}$ as a triangulated category with infinite direct sums, since the coalgebra without counit $\left(\mathcal{C} \otimes_{k} \mathcal{C}^{\mathrm{op}}\right) /\left(\mathfrak{C}^{\mathrm{ss}} \otimes_{k} \mathcal{C}^{\mathrm{ss} \text { op }}\right)$ is conilpotent [15, Section 5.5]. Therefore, the conditions of Theorem 3.5. C are satisfied for the CDG-algebra $B$.

4.7. Noetherian CDG-ring. Let $B$ be a CDG-algebra over a commutative ring $k$ and $C=\bmod _{\mathrm{fgp}}^{\mathrm{cdg}}-B$ the DG-category of right CDG-modules over $B$, projective and finitely generated as $\Gamma$-graded $B^{\#}$-modules.

Corollary A. Assume that the $\Gamma$-graded ring $B^{\#}$ is graded left Noetherian and has finite left homological dimension. Then:

(a) the natural map $\operatorname{Tor}^{C}(N, M) \longrightarrow \operatorname{Tor}^{C, I I}(N, M)$ is an isomorphism for any right $D G$-module $N$ and left $D G$-module $M$ over $C$; 
(b) the natural map $\operatorname{Ext}_{C}^{I I}(L, M) \longrightarrow \operatorname{Ext}_{C}(L, M)$ is an isomorphism for any left $D G$-modules $L$ and $M$ over $C$.

Proof. Notice that for a left Noetherian (graded) ring the weak and left homological dimensions coincide. Whenever the graded ring $B^{\#}$ is left Noetherian, the coderived category $\mathrm{D}^{\mathrm{co}}\left(B\right.$-mod $\left.{ }^{\mathrm{cdg}}\right)$ is compactly generated by CDG-modules whose underlying $\Gamma$-graded modules are finitely generated (a result of D. Arinkin, [15, Theorem 3.11.2]). Assuming additionally that the left homological dimension of $B^{\#}$ is finite, it follows easily that $\mathrm{D}^{\mathrm{co}}\left(B\right.$-mod $\left.{ }^{\mathrm{cdg}}\right)$ is compactly generated by $H^{0}\left(B-\bmod _{\mathrm{fgp}}^{\mathrm{cdg}}\right)$. (See the beginning of Section 3.5 for a brief discussion of compact generation.) It remains to apply Theorem 3.5.A(a)-(b) to deduce the assertions of the corollary.

Before formulating our next result, let us define yet another exotic derived category of CDG-modules. Given a small CDG-category $D$, the complete derived category $\mathrm{D}^{\mathrm{cmp}}\left(D\right.$-mod $\left.{ }^{\mathrm{cdg}}\right)$ of left CDG-modules over $D$ is the quotient category of the homotopy category $H^{0}\left(D-\bmod ^{\mathrm{cdg}}\right)$ by its minimal triangulated subcategory, containing $\mathrm{Ac}^{\mathrm{abs}}\left(D\right.$-mod $\left.{ }^{\mathrm{cdg}}\right)$ and closed under both infinite direct sums and products. CDG-modules belonging to the latter subcategory are called completely acyclic.

Now assume that the ring $k$ has finite weak homological dimension and the $\Gamma$ graded $k$-module $B^{\#}$ is flat. Assume further that the $\Gamma$-graded $\operatorname{ring} B^{\#}$ is both left and right Noetherian of finite homological dimension, the $\Gamma$-graded ring $B^{\#} \otimes_{k} B^{\# \text { op }}$ is graded Noetherian and the $\Gamma$-graded module $B^{\#}$ over $B^{\#} \otimes_{k} B^{\# \text { op }}$ has finite projective dimension.

Corollary B. Suppose the $C D G$-module $B$ over $B \otimes_{k} B^{\text {op }}$ belongs to the minimal triangulated subcategory of $\mathrm{D}^{\mathrm{cmp}}\left(B \otimes_{k} B^{\mathrm{op}}-\mathrm{mod}^{\mathrm{cdg}}\right)$, closed under infinite direct sums and containing all $C D G$-modules of the form $L \otimes_{k} N$, where $L$ and $N$ are a left and a right $C D G$-module over $B$ and at least one of the $\Gamma$-graded $k$-modules $L^{\#}$ and $N^{\#}$ is flat. Then for any $D G$-module $M$ over $C \otimes_{k} C^{\text {op }}$ the natural maps $H H_{*}(C, M) \longrightarrow H H_{*}^{I I}(C, M)$ and $H H^{I I, *}(C, M) \longrightarrow H H^{*}(C, M)$ are isomorphisms.

Proof. Let us check the conditions of Theorem 3.5.D. In view of [15, Theorem 3.11.2] and the discussion in Section 3.6, the triangulated subcategory with infinite direct sums in $\mathrm{D}^{\mathrm{cmp}}\left(B \otimes_{k} B^{\mathrm{op}}\right.$-mod $\left.{ }^{\mathrm{cdg}}\right)$ generated by the CDG-modules $L \otimes_{k} N$ with $L$ and $N$ as above coincides with the triangulated subcategory with infinite direct sums generated by the CDG-modules $G \otimes_{k} F$ with $G \in H^{0}\left(B\right.$-mod $\left.\operatorname{mgp}_{\mathrm{fgp}}^{\mathrm{cdg}}\right)$ and $F \in H^{0}\left(\bmod _{\mathrm{fgp}}^{\mathrm{cdg}}-B\right)$. The construction from [15, proof of Theorem 3.6] shows that there exists a closed morphism from a CDG-module $P \in H^{0}\left(B \otimes_{k} B^{\mathrm{op}}\right.$ - $\left.\bmod _{\mathrm{fgp}}^{\text {cdg }}\right)$ into the CDG-module $B$ with the cone absolutely acyclic with respect to $B \otimes_{k}$ $B^{\mathrm{op}}$ - $\bmod _{\mathrm{fpd}}^{\mathrm{cdg}}$. The triangulated subcategory with infinite direct sums generated by $H^{0}\left(B \otimes_{k} B^{\mathrm{op}}-\bmod _{\mathrm{fgp}}^{\mathrm{cdg}}\right)$ in $H^{0}\left(B \otimes_{k} B^{\mathrm{op}}\right)$ is semiorthogonal to all completely acyclic CDG-modules and maps fully faithfully to $\mathrm{D}^{\mathrm{co}}\left(B \otimes_{k} B^{\mathrm{op}}-\bmod _{\mathrm{fpd}}^{\mathrm{cdg}}\right)$ and to $\mathrm{D}^{\mathrm{cmp}}\left(B \otimes_{k} B^{\mathrm{op}}-\bmod ^{\mathrm{cdg}}\right)$ [7]. So the condition that the object $P$ is generated by the objects $G \otimes_{k} F$ can be equivalently checked in any of these triangulated categories. Notice that since the objects of $H^{0}\left(B \otimes_{k} B^{\mathrm{op}}-\bmod _{\mathrm{fgp}}^{\mathrm{cdg}}\right)$ are compact in these triangulated categories, it does not matter whether we generate $P$ from $G \otimes_{K} F$ 
using shift, cones, and infinite direct sums, or shift, cones, and passages to direct summands only.

One can drop the assumption that the $\Gamma$-graded ring $B^{\#} \otimes_{k} B^{\# \text { op }}$ is graded Noetherian by replacing the complete derived category $\mathrm{D}^{\mathrm{cmp}}\left(B \otimes_{k} B^{\mathrm{op}}\right.$-mod $\left.{ }^{\mathrm{cdg}}\right)$ with the coderived category $\mathrm{D}^{\mathrm{co}}\left(B \otimes_{k} B^{\mathrm{op}}-\bmod _{\mathrm{fpd}}^{\mathrm{cdg}}\right)$ in the formulation of Corollary B. Notice also that when the left homological dimension of $B^{\#} \otimes_{k} B^{\# \text { op }}$ is finite, all the exotic derived categories $\mathrm{D}^{\mathrm{cmp}}\left(B \otimes_{k} B^{\mathrm{op}}\right.$-mod $\left.^{\mathrm{cdg}}\right), \quad \mathrm{D}^{\mathrm{abs}}\left(B \otimes_{k} B^{\mathrm{op}}-\bmod ^{\mathrm{cdg}}\right)$, $\mathrm{D}^{\mathrm{co}}\left(B \otimes_{k} B^{\mathrm{op}}-\bmod _{\mathrm{fpd}}^{\mathrm{cdg}}\right)$, etc. coincide [15, Theorem 3.6(a)].

4.8. Matrix factorizations. Set $\Gamma=\mathbb{Z} / 2$. Let $R$ be a commutative regular local ring; suppose that $R$ is also an algebra of essentially finite type over its residue field $k$. Let $w \in R$ be a noninvertible element whose zero locus has an isolated singularity at the closed point of the spectrum of $R$. Consider the CDG-algebra $(B, d, h)$ over $k$, where $B$ is the algebra $R$ placed in degree $0, d=0$, and $h=-w$. Let $C=\bmod _{\mathrm{fgp}}^{\mathrm{cdg}}-B$ be the corresponding DG-category of right CDG-modules; its objects are conventionally called the matrix factorizations of $w$.

The computations in [18] and 2] show that the two kinds of Hochschild (co)homology for the $k$-linear DG-category $C$ are isomorphic. The somewhat stronger assertion that the natural maps $H H_{*}(C, M) \longrightarrow H H_{*}^{I I}(C, M)$ and $H H^{I I}, *(C, M) \longrightarrow$ $H H^{*}(C, M)$ are isomorphisms for any DG-module $M$ over $C \otimes_{k} C^{\text {op }}$ follows from our Corollary 4.7,B. Indeed, according to [2, Theorem 4.1 and the discussion in Section 6.1] the assumption of the corollary is satisfied in this case.

More generally, let $X$ be a smooth affine variety over a field $k$ and $R$ be the $k$-algebra of regular functions on $X$. Let $w \in R$ be such a function; consider the CDG-algebra $(B, d, h)$ constructed from $R$ and $w$ as above. Let $C=\bmod _{\mathrm{fgp}}^{\mathrm{cdg}} B$ be the DG-category of right CDG-modules over $B$, projective and finitely generated as $\Gamma$-graded $B^{\#}$-modules.

Corollary A. Assume that the morphism $w: X \backslash\{w=0\} \longrightarrow \mathbb{A}_{k}^{1}$ from the open complement of the zero locus of $w$ in $X$ to the affine line is smooth. Assume moreover that either

(a) there exists a smooth closed subscheme $Z \subset X$ such that $w: X \backslash Z \longrightarrow \mathbb{A}_{k}^{1}$ is a smooth morphism and $\left.w\right|_{Z}=0$ or

(b) the field $k$ is perfect.

Then the natural maps $H H_{*}(C, M) \longrightarrow H H_{*}^{I I}(C, M)$ and $H H^{I I, *}(C, M) \longrightarrow$ $H H^{*}(C, M)$ are isomorphisms for any $D G$-module $M$ over $C \otimes_{k} C^{\text {op }}$.

Proof. The proof is based on Corollary 4.7,B, Orlov's theorem connecting matrix factorizations with the triangulated categories of singularities [10], and some observations from the paper [8]. We will show that all objects of $H^{0}\left(B \otimes_{k} B^{\mathrm{op}}-\bmod _{\mathrm{fgp}}^{\mathrm{cdg}}\right)$ can be obtained from the objects $G \otimes_{k} F$ with $G \in H^{0}\left(B-\bmod _{\mathrm{fgp}}^{\mathrm{cdg}}\right)$ and $F \in$ $H^{0}\left(\bmod _{\mathrm{fgp}}^{\mathrm{cdg}}-B\right)$ using the operations of cone and passage to a direct summand. By Orlov's theorem, the triangulated categories $H^{0}\left(B-\bmod _{\mathrm{fgp}}^{\mathrm{cdg}}\right)$ and $H^{0}\left(\bmod _{\mathrm{fgp}}^{\mathrm{cdg}}-B\right)$ can be identified with the triangulated category $\mathrm{D}_{\text {Sing }}^{\mathrm{b}}\left(X_{0}\right)$ of singularities of the zero locus $X_{0} \subset X$ of the function $w$. Similarly, the triangulated category $H^{0}\left(B \otimes_{k}\right.$ $\left.B^{\mathrm{op}}-\bmod _{\mathrm{fgp}}^{\mathrm{cdg}}\right)$ is identified with the triangulated category $\mathrm{D}_{\text {Sing }}^{\mathrm{b}}\left(Y_{0}\right)$ of singularities 
of the zero locus $Y_{0} \subset X \times_{k} X$ of the function $w \times 1-1 \times w$ on the Cartesian product $X \times_{k} X$.

Lemma B. The equivalences of categories

$$
H^{0}\left(B-\bmod _{\mathrm{fgp}}^{\mathrm{cdg}}\right) \simeq \mathrm{D}_{\text {Sing }}^{\mathrm{b}}\left(X_{0}\right) \simeq H^{0}\left(\bmod _{\mathrm{fgp}}^{\mathrm{cdg}}-B\right)
$$

and $H^{0}\left(B \otimes_{k} B^{\mathrm{op}}-\bmod _{\mathrm{fgp}}^{\mathrm{cdg}}\right) \simeq \mathrm{D}_{\text {Sing }}^{\mathrm{b}}\left(Y_{0}\right)$ transform the external tensor product functor $H^{0}\left(B-\bmod _{\mathrm{fgp}}^{\mathrm{cdg}}\right) \times H^{0}\left(\bmod _{\mathrm{fgp}}^{\mathrm{cdg}}-B\right) \longrightarrow H^{0}\left(B \otimes_{k} B^{\mathrm{op}}-\bmod _{\mathrm{fgp}}^{\mathrm{cdg}}\right)$ into the functor $\mathrm{D}_{\text {Sing }}^{\mathrm{b}}\left(X_{0}\right) \times \mathrm{D}_{\text {Sing }}^{\mathrm{b}}\left(X_{0}\right) \longrightarrow \mathrm{D}_{\text {Sing }}^{\mathrm{b}}\left(Y_{0}\right)$ induced by the composition of the external tensor product of coherent sheaves on two copies of $X_{0}$ and the direct image under the closed embedding $X_{0} \times_{k} X_{0} \longrightarrow Y_{0}$.

Proof. Rather than checking the assertion of the lemma for Orlov's cokernel functor $\Sigma: H^{0}\left(B-\bmod _{\mathrm{fgp}}^{\mathrm{cdg}}\right) \longrightarrow \mathrm{D}_{\text {Sing }}^{\mathrm{b}}\left(X_{0}\right)$, one can use the construction of the inverse functor $\Upsilon: \mathrm{D}_{\text {Sing }}^{\mathrm{b}}\left(X_{0}\right) \longrightarrow H^{0}\left(B\right.$ - $\left.\bmod _{\mathrm{fgp}}^{\mathrm{cdg}}\right)$ given in [16], for which the desired compatibility is easy to establish. Alternatively, it suffices to use the result of 8 , Lemma 2.18].

Let $\mathrm{D}^{\mathrm{abs}}\left(B-\bmod _{\mathrm{fg}}^{\mathrm{cdg}}\right)$ denote the absolute derived category of left CDG-modules over $B$ whose underlying $\Gamma$-graded $B^{\#}$-modules are finitely generated. The notation $\mathrm{D}^{\mathrm{abs}}\left(\bmod _{\mathrm{fg}}^{\mathrm{cdg}}-B\right)$ for right CDG-modules will have a similar meaning. Then the external tensor product of finitely generated CDG-modules induces a functor $\mathrm{D}^{\mathrm{abs}}\left(B-\mathrm{mod}_{\mathrm{fg}}^{\mathrm{cdg}}\right) \times \mathrm{D}^{\mathrm{abs}}\left(\bmod _{\mathrm{fg}}^{\mathrm{cdg}}-B\right) \longrightarrow \mathrm{D}^{\mathrm{abs}}\left(B \otimes_{k} B^{\mathrm{op}}-\bmod _{\mathrm{fg}}^{\mathrm{cdg}}\right)$. Furthermore, the natural functor $H^{0}\left(B-\bmod _{\mathrm{fgp}}^{\mathrm{cdg}}\right) \longrightarrow \mathrm{D}^{\mathrm{abs}}\left(B-\bmod _{\mathrm{fg}}^{\mathrm{cdg}}\right)$ is an equivalence of categories, since $B^{\#}$ is Noetherian of finite homological dimension.

Let $M \in H^{0}\left(B-\bmod _{\mathrm{fgp}}^{\mathrm{cdg}}\right)$. The direct image of the corresponding coherent sheaf $\Sigma(M)$ on $X_{0}$ under the closed embedding $X_{0} \longrightarrow X$ can be viewed as an object of $\mathrm{D}^{\mathrm{abs}}\left(B-\bmod _{\mathrm{fg}}^{\mathrm{cdg}}\right)$. It is clear from the above-mentioned lemma from [8] that this object is naturally isomorphic to the image of $M$ in $\mathrm{D}^{\mathrm{abs}}\left(B-\bmod _{\mathrm{fg}}^{\mathrm{cdg}}\right)$. Let $N \in H^{0}\left(\bmod _{\mathrm{fgp}}^{\mathrm{cdg}}-B\right)$. Then the coherent sheaf $\Sigma(N)$ on $X_{0}$, viewed as an object of $\mathrm{D}^{\mathrm{abs}}\left(\bmod _{\mathrm{fg}}^{\mathrm{cdg}}-B\right)$, is isomorphic to $N$.

Since the external tensor product is well-defined on the absolute derived categories of finitely generated CDG-modules, it follows that the coherent sheaf $\Sigma(M) \otimes_{k}$ $\Sigma(N)$ on $X_{0} \times_{k} X_{0}$, viewed as an object of $\mathrm{D}^{\mathrm{abs}}\left(B \otimes_{k} B^{\mathrm{op}}-\bmod _{\mathrm{fg}}^{\mathrm{cdg}}\right)$, is isomorphic to $M \otimes_{k} N$. Applying the same lemma from [8] again, we conclude that $\Sigma\left(M \otimes_{k} N\right) \simeq \Sigma(M) \bigotimes_{k} \Sigma(N)$ in $\mathrm{D}_{\text {Sing }}^{\mathrm{b}}\left(Y_{0}\right)$. The assertion of Lemma B is proven.

Now we can finish the proof of the corollary. Recall that in case (a) we have a closed subvariety $Z \subset X_{0}$; in case (b), let $Z=X_{0}$ (or any closed subvariety of $X_{0}$ such that the morphism $w: X \backslash Z \longrightarrow \mathbb{A}_{k}^{1}$ is smooth). It suffices to show that the external tensor products of coherent sheaves on two copies of $Z$, considered as coherent sheaves on $Y_{0}$, generate the triangulated category of singularities of $Y_{0}$.

The open complement to $Z \times Z$ in $Y_{0}$ is a smooth variety. Indeed, we have $Y_{0}=X \times_{\mathbb{A}_{k}^{1}} X$. The complement to $Z \times Z$ in $Y_{0}$ is covered by its open subschemes $(X \backslash Z) \times_{\mathbb{A}_{k}^{1}} X$ and $X \times_{\mathbb{A}_{k}^{1}}(X \backslash Z)$, which are both smooth, since $X$ is smooth over $k$ and $X \backslash Z$ is smooth over $\mathbb{A}_{k}^{1}$. By [11, Proposition 2.7] (see also [8, Theorem 3.5] and [9, Theorem 2.1.5 and/or Lemma 2.6]), it follows that the triangulated category of singularities of $Y_{0}$ is generated by coherent sheaves on $Z \times{ }_{k} Z$. 
It remains to show that the derived category of coherent sheaves on $Z \times_{k} Z$ is generated by the external tensor products of coherent sheaves on the Cartesian factors. This is true, at least, (a) for any smooth affine scheme $Z$ of finite type over a field $k$, and (b) for any affine scheme $Z$ of finite type over a perfect field $k$ (the affineness assumption can be weakened, of course). Case (a) is clear, since $Z \times_{k} Z$ is (affine and) regular of finite Krull dimension. In case (b), any reduced scheme of finite type over $k$ contains an open dense smooth subscheme [4, Corollaire (17.15.13)], and [8, (proof of) Theorem 3.7] applies.

When $X$ contains connected components on which $w$ is identically zero, Orlov's theorem is not applicable. On such components, one has to consider the $\mathbb{Z} / 2$ graded derived category of coherent sheaves in place of the triangulated category of singularities of the zero locus. Otherwise, the above argument remains unchanged.

4.9. Trivial counterexample. The two kinds of Hochschild (co)homology of DGalgebras cannot always be isomorphic for very general reasons.

The Hochschild homology and cohomology of the first kind $H H_{*}(A)$ and $H H^{*}(A)$ of a DG-algebra $A$ are invariant with respect to quasi-isomorphisms of DG-algebras (see Section 2.4). On the other hand, the Hochschild homology and cohomology of the second kind $H H_{*}^{I I}(A)$ and $H H^{I I, *}(A)$ are invariant with respect to isomorphisms of DG-algebras in the category of CDG-algebras (since the Hochschild (co)homology of the second kind are generally functorial with respect to CDGfunctors; see Sections 2.2 2.4). These are two incompatible types of invariance properties. Indeed, any two DG-algebras over a field can be connected by a chain of tranformations, some of which are quasi-isomorphisms and the other are CDGisomorphisms [15, Examples 9.4].

Here is a rather trivial example of a CDG-algebra $B$ over a field $k$ such that for the corresponding DG-category $C=\bmod _{\mathrm{fgp}}^{\mathrm{cdg}}-B$ the two kinds of Hochschild (co)homology are different. This example also shows that one cannot drop the conditions on the differential $d$ and curvature $h$ of the CDG-coalgebra $\mathcal{C}$ in Corollary 4.6. B, nor can one drop the condition on the critical values of the potential $w$ in Corollary 4.8, A.

Set $\Gamma=\mathbb{Z} / 2$ and $(B, d, h)=(k, 0,1)$. So $B$ is the $k$-algebra $k$ placed in the grading $0 \bmod 2$ and endowed with the zero differential and a nonzero curvature element. Then any CDG-module over $B$ is contractible, any one of the two components of the differential of a CDG-module being its contracting homotopy. So the DG-category $C$ is quasi-equivalent to zero, hence $H H_{*}(C)=0=H H^{*}(C)$.

On the other hand, the CDG-algebra $B \otimes_{k} B^{\text {op }}$ is simply the $\mathbb{Z} / 2$-graded $k$-algebra $k$ with the zero differential and curvature. The CDG-module $B$ over $B \otimes_{k} B^{\text {op }}$ is the $\mathbb{Z} / 2$-graded $k$-module $k$ concentrated in degree $0 \bmod 2$. So $\operatorname{Tor}^{B \otimes_{k} B^{\text {op }}}(B, B) \simeq$ $\operatorname{Tor}^{B \otimes_{k} B^{\mathrm{op}}, I I}(B, B) \simeq k \simeq \operatorname{Ext}_{B \otimes_{k} B^{\mathrm{op}}}^{I I}(B, B) \simeq \operatorname{Ext}_{B \otimes_{k} B^{\mathrm{op}}}(B, B)$. We conclude that $H H_{*}^{I I}(B) \simeq k \simeq H H^{I I, *}(B)$ and, by the isomorphisms (45) from Section 2.6. $H H_{*}^{I I}(C) \simeq k \simeq H H^{I I, *}(C)$.

4.10. Direct sum formula. Set $\Gamma=\mathbb{Z} / 2$. Let $k$ be a commutative ring and $B$ be a small $k$-linear CDG-category such that all $\Gamma$-graded $k$-modules of morphisms in $B^{\#}$ are $k$-flat. Given a constant $c \in k$, denote by $B_{(c)}$ the $k$-linear CDG-category obtained from the CDG-category $B$ by adding $c$ to all the curvature elements in $B$. Then there is a natural (strict) isomorphism $B_{(c)} \otimes_{k} B_{(c)}^{\mathrm{op}} \simeq B \otimes_{k} B^{\mathrm{op}}$, hence a 
natural isomorphism between the DG-categories of CDG-modules over $B_{(c)} \otimes_{k} B_{(c)}^{\text {op }}$ and $B \otimes_{k} B^{\text {op }}$. This isomorphism transforms the diagonal CDG-bimodule $B_{(c)}$ over $B_{(c)} \otimes_{k} B_{(c)}^{\text {op }}$ to the diagonal CDG-bimodule $B$ over $B \otimes_{k} B^{\text {op }}$.

Therefore, we have natural isomorphisms

$$
H H_{*}^{I I}\left(B_{(c)}\right) \simeq H H_{*}^{I I}(B) \text { and } H H^{I I, *}\left(B_{(c)}\right) \simeq H H^{I I, *}(B),
$$

and consequently similar isomorphisms for the Hochschild (co)homology of the second kind of the DG-categories $C=\bmod _{\mathrm{fgp}}^{\mathrm{cdg}}-B$ and $C_{(c)}=\bmod _{\mathrm{fgp}}^{\mathrm{cdg}}-B_{(c)}$. Hochschild (co)homology of the first kind of the DG-categories $C$ and $C_{(c)}$ are not isomorphic in general (and in fact can be entirely unrelated, as the following example illustrates).

Let $k$ be an algebraically closed field of characteristic $0, X$ be a smooth affine variety over $k$, and $w$ be a regular function on $X$. Let $B$ be the CDG-algebra associated with $X$ and $w$ as in Section 4.8. The function $w$ has a finite number of critical values $c_{i} \in k$. When $c$ is not a critical value, the Hochschild (co)homology of the first kind $H H_{*}\left(C_{(c)}\right)$ and $H H^{*}\left(C_{(c)}\right)$ vanish, since the category $H^{0}\left(C_{(c)}\right)$ does. We have natural maps

$$
H H_{*}\left(C_{\left(c_{i}\right)}\right) \longrightarrow H H_{*}^{I I}\left(C_{\left(c_{i}\right)}\right) \simeq H H_{*}^{I I}(C)
$$

and

$$
H H^{I I, *}(C) \simeq H H^{I I, *}\left(C_{\left(c_{i}\right)}\right) \longrightarrow H H^{*}\left(C_{\left(c_{i}\right)}\right) .
$$

Corollary. The induced maps

$$
\bigoplus_{i} H H_{*}\left(C_{\left(c_{i}\right.}\right) \longrightarrow H H_{*}^{I I}(C)
$$

and

$$
H H^{I I, *}(C) \longrightarrow \bigoplus_{i} H H^{*}\left(C_{\left(c_{i}\right)}\right)
$$

are isomorphisms.

Proof. It follows from the spectral sequence computation for the Hochschild (co)homology of the second kind in [1, proof of Theorem 4.2(b)] (see also [8, Lemma 3.3]) that $H H_{*}^{I I}(C)$ and $H H^{I I}, *(C)$ decompose into direct sums over the critical values of $w$. More precisely, let $X_{c_{i}} \subset X$ denote the closed subscheme defined by the equation $w=c_{i}$, and let $X_{i}^{\prime} \subset X$ denote the open complement to the union of $X_{c_{j}}$ over all $j \neq i$. Clearly, $X_{i}^{\prime}$ is an affine scheme.

Let $B_{i}^{\prime}$ denote the CDG-algebra associated with $X_{i}^{\prime}$ and $w$ as above, and let $C_{i}^{\prime}=\bmod _{\mathrm{fgp}}^{\mathrm{cdg}}-B_{i}^{\prime}$ be the related DG-category of CDG-modules. Then the natural map $H H_{*}^{I I}(C) \longrightarrow \bigoplus_{i} H H_{*}^{I I}\left(C_{i}^{\prime}\right)$ induced by the DG-functors $C \longrightarrow C_{i}^{\prime}$ is an isomorphism, since the natural map $H H_{*}^{I I}(B) \longrightarrow \bigoplus_{i} H H_{*}^{I I}\left(B_{i}^{\prime}\right)$ is.

Furthermore, the diagonal CDG-bimodule $B_{i}^{\prime}$ over $B_{i}^{\prime}$ can be considered as a CDG-bimodule over $B$ by means of the strict CDG-functor $B \longrightarrow B_{i}^{\prime}$, and similarly the diagonal DG-bimodule $C_{i}^{\prime}$ over $C_{i}^{\prime}$ can be considered as a DG-bimodule over $C$. The CDG-bimodule $B_{i}^{\prime}$ over $B$ corresponds to the DG-bimodule $C_{i}^{\prime}$ over $C$ under the

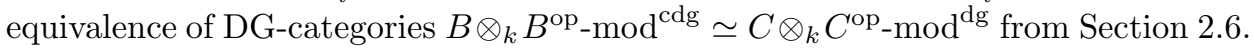
The natural maps $H H^{I I, *}\left(C_{i}^{\prime}\right) \longrightarrow H H^{I I, *}\left(C, C_{i}^{\prime}\right)$ are isomorphisms, since the natural maps $H H^{I I, *}\left(B_{i}^{\prime}\right) \longrightarrow H H^{I I, *}\left(B, B_{i}^{\prime}\right)$ are, and the map $H H^{I I, *}(C) \longrightarrow$ $\bigoplus_{i} H H^{I I, *}\left(C, C_{i}^{\prime}\right)$ is an isomorphism, for a similar reason.

On the other hand, Orlov's theorem [10] implies that the DG-functor $C_{\left(c_{i}\right)} \longrightarrow$ $C_{i\left(c_{i}\right)}^{\prime}$, where $C_{i\left(c_{i}\right)}^{\prime}=\bmod _{\mathrm{fgp}}^{\mathrm{cdg}}-B_{i\left(c_{i}\right)}^{\prime}$ and $B_{i\left(c_{i}\right)}^{\prime}$ is the CDG-algebra associated with 
the variety $X_{i}^{\prime}$ and the function $w-c_{i}$, is a quasi-equivalence. Thererefore, the natural maps $H H_{*}\left(C_{\left(c_{i}\right)}\right) \longrightarrow H H_{*}\left(C_{i\left(c_{i}\right)}^{\prime}\right)$ are isomorphisms, as are the natural maps $H H^{*}\left(C_{\left(c_{i}\right)}\right) \longrightarrow H H^{*}\left(C_{\left(c_{i}\right)}, C_{i\left(c_{i}\right)}^{\prime}\right) \longleftarrow H H^{*}\left(C_{i\left(c_{i}\right)}^{\prime}\right)$. Besides, one has $H^{0}\left(C_{i\left(c_{j}\right)}\right)=0$, hence $H H_{*}\left(C_{i\left(c_{j}\right)}\right)=0=H H^{*}\left(C_{i\left(c_{j}\right)}\right)$, for all $i \neq j$.

The isomorphisms (54) (55) now follow from Corollary 4.8 A applied to the varieties $X_{i}^{\prime}$ with the functions $w-c_{i}$ on them and commutativity of the natural diagrams.

\section{REFERENCES}

[1] A. Caldararu, J. Tu. Curved A-infinity algebras and Landau-Ginzburg models. Electronic preprint arXiv:1007.2679 [math.KT], 42 pp.

[2] T. Dyckerhoff. Compact generators in categories of matrix factorizations. Electronic preprint arXiv:0904.4713 [math.AG], 43 pp.

[3] E. Getzler, J. D. S. Jones. $A_{\infty}$-algebras and the cyclic bar complex. Illinois Journ. of Math. 34, \#2, 1990. MR1046565 (91e:19001)

[4] A. Grothendieck, J. Dieudonné. Éléments de géométrie algébrique IV. Étude locale des schémas et des morphismes de schémas, Quatrième partie. Publ. Math. IHES 32, pp. 5361, 1967. MR0238860 (39:220)

[5] D. Husemoller, J. C. Moore, J. Stasheff. Differential homological algebra and homogeneous spaces. Journ. Pure Appl. Algebra 5, pp. 113-185, 1974. MR0365571 (51:1823)

[6] B. Keller. Deriving DG-categories. Ann. Sci. École Norm. Sup. (4) 27, \#1, pp. 63-102, 1994. MR $1258406(95 \mathrm{e}: 18010)$

[7] B. Keller, W. Lowen, P. Nicolás. On the (non)vanishing of some "derived" categories of curved dg algebras. Journ. Pure Appl. Algebra 214, \#7, pp. 1271-1284, 2010. MR2587002 (2011b:16041)

[8] K. Lin, D. Pomerleano. Global matrix factorizations. Electronic preprint arXiv:1101.5847 [math.AG], 15 pp.

[9] A. Neeman. The Grothendieck duality theorem via Bousfield's techniques and Brown representability. Journ. Amer. Math. Soc. 9, pp. 205-236, 1996. MR1308405 (96c:18006)

[10] D. Orlov. Triangulated categories of singularities and D-branes in Landau-Ginzburg models. Proc. Steklov Math. Inst. 246, \#3, pp. 227-248, 2004. MR2101296 (2006i:81173)

[11] D. Orlov. Formal completions and idempotent completions of triangulated categories of singularities. Advances in Math. 226, \#1, pp. 206-217, 2011. MR2735755

[12] A. Polishchuk, A. Vaintrob. Chern characters and Hirzebruch-Riemann-Roch formula for matrix factorizations. Electronic preprint arXiv:1002.2116 [math.AG], 45 pp.

[13] L. Positselski. Nonhomogeneous quadratic duality and curvature. Funct. Anal. Appl. 27, \#3, pp. 197-204, 1993. MR:1250981 (95h:16041)

[14] L. Positselski. Homological algebra of semimodules and semicontramodules: Semi-infinite homological algebra of associative algebraic structures. Appendix $\mathrm{C}$ in collaboration with D. Rumynin; Appendix D in collaboration with S. Arkhipov. Monografie Matematyczne, vol. 70, Birkhäuser/Springer Basel, 2010, xxiv+349 pp. MR2723021

[15] L. Positselski. Two kinds of derived categories, Koszul duality, and comodule-contramodule correspondence. Memoirs Amer. Math. Soc. 212, \#996, 2011, v+133 pp.arXiv:0905.2621 [math.CT]

[16] L. Positselski. Coherent analogues of matrix factorizations and relative singularity categories. Electronic preprint arXiv:1102.0261 [math.CT], 16 pp.

[17] L. Positselski, A. Vishik. Koszul duality and Galois cohomology. Math. Research Letters 2, \#6, pp. 771-781, 1995. MR1362968 (97b:12008)

[18] E. Segal. The closed state space of affine Landau-Ginzburg B-models. Electronic preprint arXiv:0904.1339 [math.AG], 30 pp.

[19] A. Schwarz. Noncommutative supergeometry, duality and deformations. Nuclear Physics B 650, pp. 475-496, 2003. MR.1952770 (2004e:58012)

[20] G. Tabuada. Une structure de catégorie de modèles de Quillen sur la catégorie des dgcatégories. A Quillen model structure on the category of dg categories. Comptes Rendus Acad. Sci. Paris 340, \#1, pp. 15-19, 2005. MR2112034(2005h:18033) 
[21] B. Toën. The homotopy theory of dg-categories and derived Morita theory. Inventiones Math. 167, \#3, pp. 615-667, 2007. MR2276263(2008a:18006)

[22] J. Tu. Matrix factorizations via Koszul duality. Electronic preprint arXiv:1009.4151 [math.AG], $53 \mathrm{pp}$.

Department of Mathematics, University of Oregon, Eugene, Oregon 97403

E-mail address: apolish@uoregon.edu

Department of Mathematics, National Research University "Higher School of Economics", Moscow 117312, Russia - And - Sector of Algebra and Number Theory, Institute for Information Transmission Problems, Moscow 127994, Russia

E-mail address: posic@mccme.ru 